University of New Mexico

UNM Digital Repository

Chemistry ETDs

Electronic Theses and Dissertations

Summer 7-21-1977

Comparisons Between Oriented Film and Solution Tertiary

Structure of Various Nucleic Acids

Douglas R. C. Priore

Follow this and additional works at: https://digitalrepository.unm.edu/chem_etds

Part of the Organic Chemistry Commons, and the Physical Chemistry Commons 


\section{THE UNIVERSITY OF NEW MEXICO}

\section{ALBUQUERQUE, NEW MEXICO 87131}

\section{Policy on Use of Theses and Dissertations}

Unpublished theses and dissertations accepted for master's and doctor's degrees and deposited in the University of New Mexico Library are open to the public for inspection and reference work. They are to be used only with due regard to the rights of the authors. The work of other authors should always be given full credit. Avoid quoting in amounts, over and beyond scholarly needs, such as might impair or destroy the property rights and financial benefits of another author.

To afford reasonable safeguards to authors, and consistent with the above principles, anyone quoting from theses and dissertations must observe the following conditions:

1. Direct quotations during the first two years after completion may be made only with the written permission of the author.

2. After a lapse of two years, theses and dissertations may be quoted without specific prior permission in works of original scholarship provided appropriate credit is given in the case of each quotation.

3. Quotations that are complete units in themselves (e.g., complete chapters or sections) in whatever form they may be reproduced and quotations of whatever length presented as primary material for their own sake (as in anthologies or books of readings) ALWAYS require consent of the authors.

4. The quoting author is responsible for determining "fair use" of material he uses.

This thesis/dissertation by Douglas R.C. Priore has been used by the following persons whose signatures attest their acceptance of the above conditions. (A library which borrows this thesis/dissertation for use by its patrons is expected to secure the signature of each user.)

NAME AND ADDRESS

DATE 
This dissertation, directed and approved by the candidate's committee, has been accepted by the Graduate Committee of The University of New Mexico in partial fulfillment of the requiremints for the degree of

Doctor of Philosophy

Comparisons Between Oriented Film and Solution Torte

Douglas R. C. Prior

Candidate

Chemistry

Department
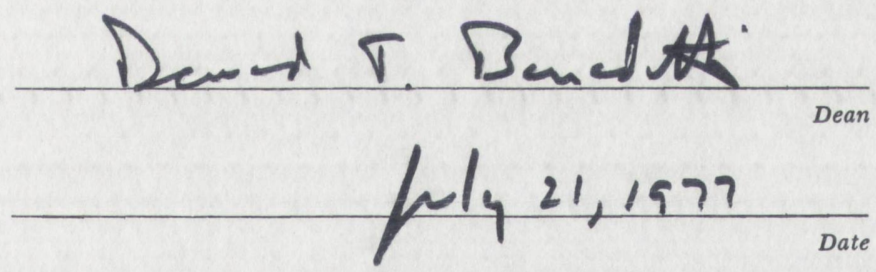

Committee

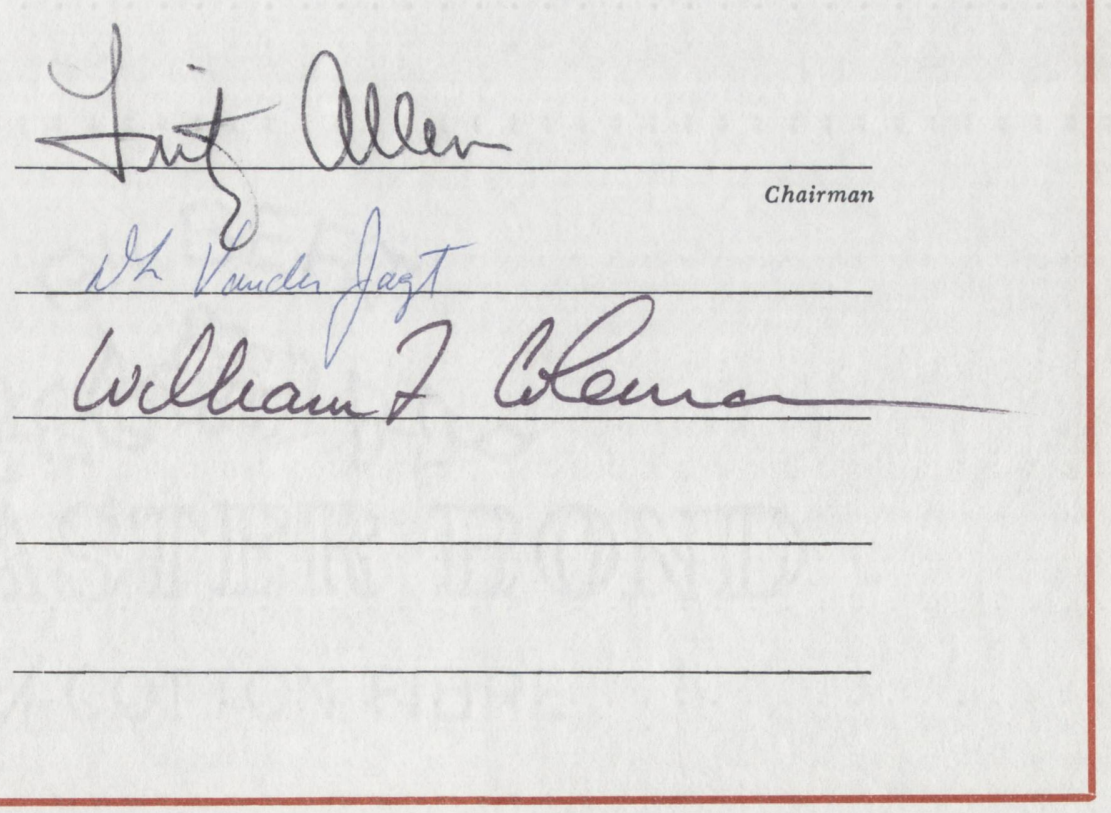




\title{
COMPARISONS BETWEEN ORIENTED FILM AND SOLUTION TERTIARY STRUCTURE OF VARIOUS NUCLEIC ACIDS
}

\author{
BY \\ DOUGLAS R. C. PRIORE \\ B.A., Rutgers University, 1972 \\ M.S., The University of New Mexico, 1974
}

\section{DISSERTATION}

Submitted in Partial Fulfillment of the

Requirements for the Degree of

Doctor of Philosophy in Chemistry in the Graduate School of

The University of New Mexico

Albuquerque, New Mexico

August, 1977 
Dr. Fritz Allen, as the author's advisor, was an incredible help, especially in his encouragement and incessant willingness to discuss all phases of this thesis. His concern evinced itself in many ways, even to the extent of that of a good friend, for which the author is exceedingly grateful.

It is also with indebtedness that fellow graduate student Bill Rahe is acknowledged. His perserverance with the E.D. instrument throughout its final implementation could not be more appreciated.

Then there must also be noted fellow grad students, Mike McIwain and R. J. Fraatz, whose friendships were also a source of much inspiration. Additionally during the earlier years of work, that were later aborted, the author recalls Dr. William Coleman's help with crystal spectra and expresses his thanks. Along these same lines, ie., while working with tRNA, the many useful and stimulating talks with Dr. Gary Quigley of M.I.T. are gratefully recognized. Finally the curiously sardonic prodding of Dr. Guido Daub and the goodness and personality of the entire chemistry department is acknowledged.

(And through it all comes an accepting attitude that has fostered a desire to further the better things that can and have been found.) 
COMPARISONS BETWEEN ORIENTED FILM AND SOLUTION TERTIARY STRUCTURE OF VARIOUS NUCLEIC ACIDS

BY

Douglas R. C. Priore

ABSTRACT OF DISSERTATION

Submitted in Partial Fulfillment of the

Requirements for the Degree of

Doctor of Philosophy in Chemistry

in the Graduate School of

The University of New Mexico

Albuquerque, New Mexico

August, 1977 


\title{
COMPARISONS BETWEEN ORIENTED FILM AND SOLUTION TERTIARY STRUCTURE OF VARIOUS NUCLEIC ACIDS
}

\author{
Douglas R. C. Priore, Ph.D. \\ Department of Chemistry \\ The University of New Mexico, 1977
}

It was desired to help establish whether or not the tertiary structure of a biomolecule is the same in a crystal (or an oriented film) as it is in solution. Generally it is accepted that the structures in these diverse environments are similar, if not identical. By comparing the extinction coefficients obtained from oriented fil.n data to those obtained from solution data it was hoped that this question might further be resolved. To this end an electric dichroism instrument built in our lab was used to measure the dichroic signal so as to calculate, with the help of the ultraviolet absorption spectra of the solution, extinction coefficients in the parallel and perpendicular directions. These were then compared to data from the oriented film to determine whether the two sets of data could be rotated into each other using a computer program based on a linear programming method.

For the four nucleic acids studied, the two sets of data were found to be equivalent and required rotation through an angle of zero degrees to force this equivalence. It was also found that flow dichroism data and electric dichroism data were equivalent. It was thus established 
that the dipole moment is along the helical axis in the nucleic acids. Additionally it was shown that the physical, hydrodynamical, and electrical axes are symmetrical and coincident.

Decay curves for relaxation from the transient orienting phenomena were plotted and rotary diffusion coefficients were calculated using the peeling method for a two component system to yield both a slow and a fast relaxing component. Relaxation times were also calculated. And finally it was observed that the electric dichroism instrument functioned correctly in that. it could reproduce literature values. 
TABLE OF CONTENTS

Section

Page

I. BACKGROUND AND EXPERIMENTAL BASIS

1. Introduction

2. tRNA in Solution and as a Crystal

3. Orthorhombic and Monoclinic Crystal Form Comparison

4. Oriented Film Spectra of Nucleic Acids

5. Flow Dichroism

6. Comparisons Between Flow Dichroism Spectra

7. Oriented Film Spectra of Polynucleotides

1

1

8

II. THEORETICAL BASIS

III. COMPUTER TREATMENT

IV. THEORY OF ELECTRIC DICHROISM

V. ELECTRIC DICHROISM INSTRUMENT AND TECHNIQUES

VI. EXPERIMENTAL SECTION

1. Results with tRNA

2. Results with DNA

56

3. Results with Polynucleotides

4. Comparison Calculations with Flow Dichroism

5. Results for Relaxation of Electric Dichroism

VII. DISCUSSION OF RESULTS

Appendix A. Main Program with LINIT 75

Appendix B. Degenerate of Main Program 92

Appendix C. Main Program with SIMPLX 94

$\begin{array}{ll}\text { Appendix D. SIGNAL } & 108\end{array}$

Appendix E. Film Data 112

Electric Dichroism and UV Absorption Data

Appendix F. Detailed Block Diagram of Electric Dichroism Instrument

Appendix G. Graph of Photoelastic Modulator Amplitude versus Wavelength 
viii 


\section{LIST OF FIGURES}

Figure

Page

1. tRNA Cloverleaf Structure

2. tRNA tertiary structure

3. Ultraviolet Absorption curves of Calf Thymus DNA under different conditions of humidity.

4. Polarized Absorption spectra for film of T2 DNA at increasing relative humidities.

5. Polarized Absorption spectra for film of $T 2$ DNA at decreasing relative humidities.

6. Humidity history of film

7. Ultraviolet absorption spectra of oriented film of poly $\mathrm{C}$ at $50 \%$ relative humidity

8. Ultraviolet absorption spectra of oriented film of poly (dAT) at $60 \%$

9. $\mathrm{X}, \mathrm{Y}$, and $\mathrm{Z}$ polarization in a crystal

10. Model and parameter diagram

11. Simple Block Diagram of Electric Dichroism Instrument

12. Typical Electric Dichroism Signal with Pulse

13. Steady State Electric Dichroism of T2 DNA

14. Steady State Electric Dichroism of Calf Thymus DNA

15. Steady State Electric Dichroism of poly (dAT) copolymer

16. Steady State Electric Dichroism of polycytidylic acid

17. Relaxation plot of T2 DNA

18. Relaxation plot of Calf Thymus DNA 68

19. Relaxation plot of poly (dAT-dAT)

20. Steady State dichroism däta graphed versus $1 / \mathrm{E}$ 
21. Detailed Block Diagram of Electric Dichroism Instrument

22. Photoelastic Modulator Amplitude versus Wavelength 


\section{LIST OF TABLES}

Table

Page

1. Results from computer treatment of data for indicated nucleic acids

2. Rotational Diffusion Coefficients for Calf Thymus DNA 


\section{BACKGROUND AND EXPERIMENTAL BASIS}

\section{Introduction}

For several years the precise tertiary structure of transfer ribonucleic acid ( $t R N A$ ) has been a problem that many labs have been attempting to unravel and definitively elucidate. The reason for this lies in the fact that tRNA is an important biomolecule in that it transfers information between nucleic acid and protein sequences. The primary and secondary structure are known for many tRNA molecules. There exists a good basis for further explaining the function or mode of action of the molecule in structural terms from its tertiary structure; that is, just how the molecule winds about itself or the conformation it is in as it transfers its information to the ribosome.

A considerable number of different methods have been used in various labs to obtain this type of information. Most notable among these is the technique of $x$-ray diffraction. A group at Massachusetts Institute of Technology working independently and jointly with people from Duke University has made continual improvements in their resolution $(1-7)$ as has another group in Cambridge $(8,9,2,10)$. The current resolution is to $2.5 \mathrm{~A}$, a resolution obtained through a method of isomorphous replacement and refinement involving the use of six heavy metal atom derivatives for the concomitantly improved electron density map. So far, the extent of x-ray diffraction crystal 
work has been performed on single crystals of both monoclinic and orthorhombic forms of crystalline yeast tRNA ${ }^{\text {Phe }}$. Other techniques for structural observation of tRNA also involve use of instrumentation, such as NMR (11, 12, 13, 14), Raman Spectroscopy $(15,16), \operatorname{ESR}(17)$, Circular Dichroism (18, 19, 20, 21, 22, 23), Fluourescence Detected Circular Dichroism (24), and Luminescence (25), while studies on kinetics (26), aminoacylation $(27,28,29,30$, 31), and various perturbations of the environment have furthered the knowledge of tRNA mode of action and recognition of sites.

Through the various structural probes a rather specific understanding and picture of the tRNA molecule has developed. The shape of the ribose-phosphate backbone is known, thus defining where the loops and stems are oriented within the three dimensional structure and what network of bonds helps to maintain this structure. Stacking of the planar purines and pyrimidines stabilizes the structure (2) while the general tertiary structure is described by hydrogen-bonding interaction which requires great specificity to maintain.

The binding of the tRNA molecule is suggested to be accompanied by structural rearrangements in the tRNA exposing ribosomal binding sites and the interaction between codon and anticodon induces conformational changes in the tRNA (32). However these interactions are difficult to fully describe especially since the tertiary 


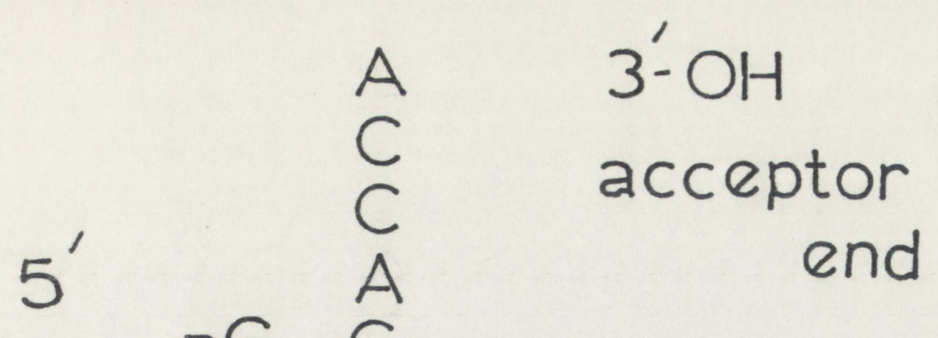

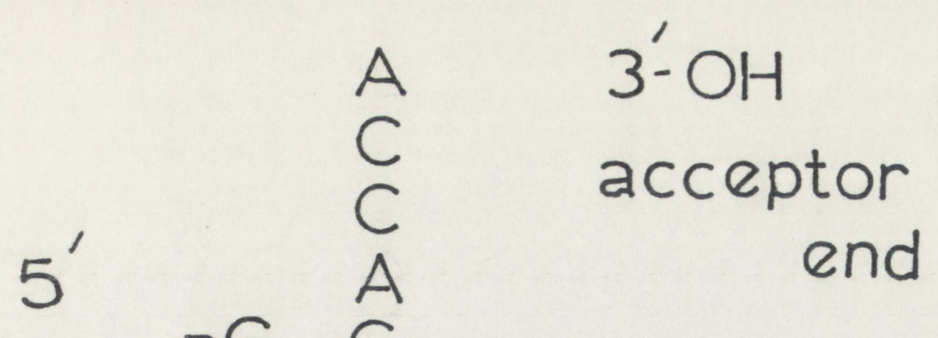

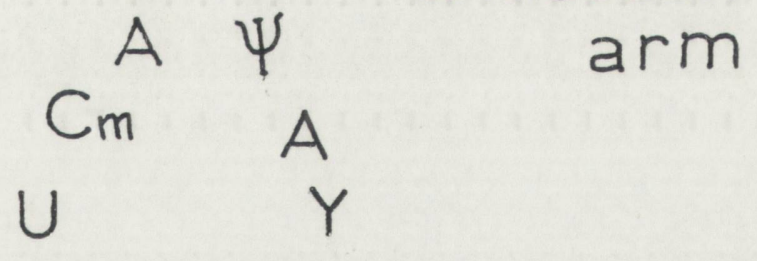

tRNA Phe

cloverleaf structure

Figure 1. (33) 


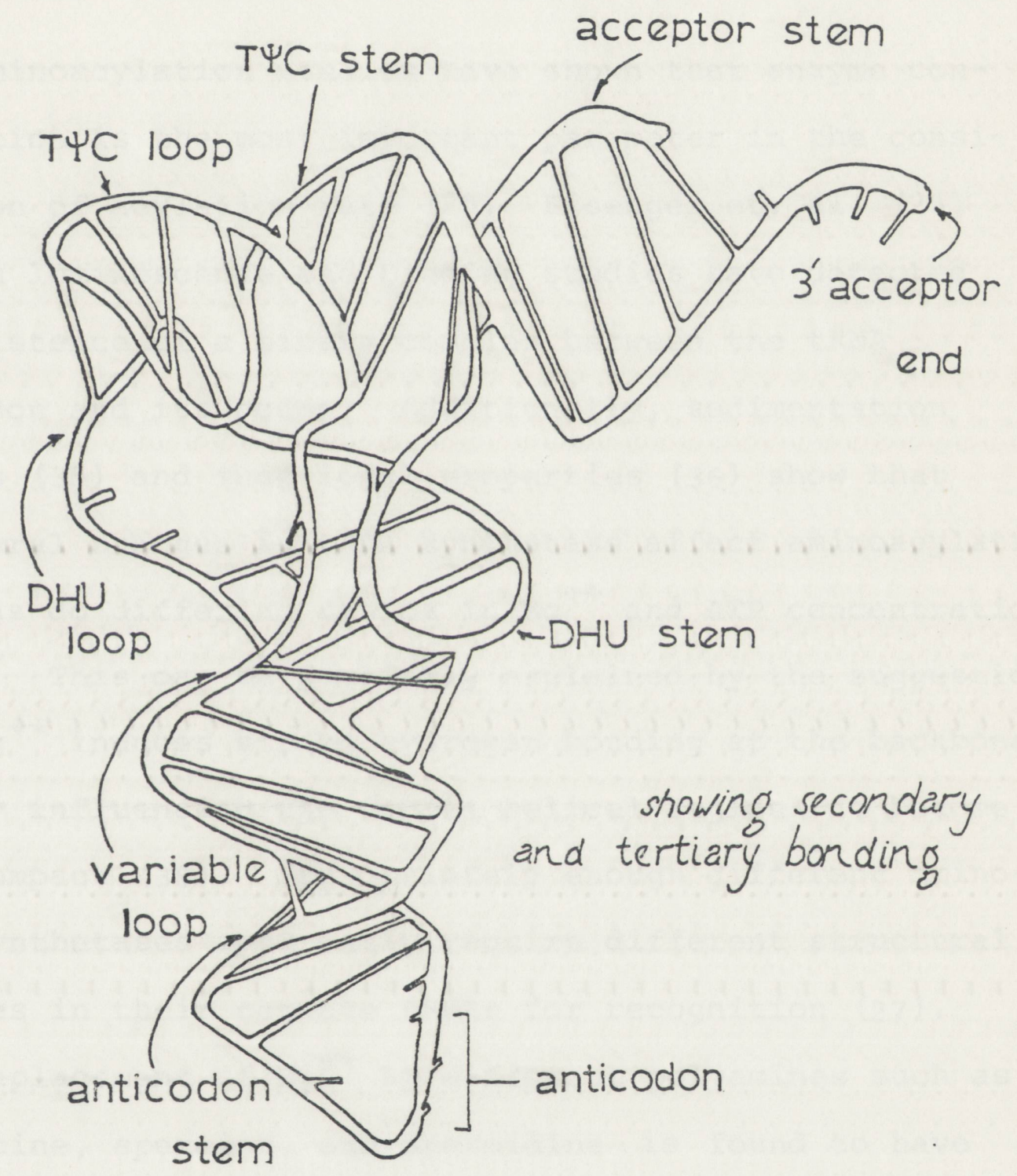

Figure 2. (4)

tRNA tertiary structure 
structure apparently influences this binding and determines the degree of preference. Often changes in $\mathrm{pH}$ are seen to also affect the degree and rate of binding of codon and anticodon since tertiary structure is thereby altered (34, 35).

Aminoacylation studies have shown that enzyme concentration is the most important parameter in the consideration of acylation rate (28) Eisenger et. al. (25) through luminescence and binding studies have detected the existence of a binary complex between the tRNA anticodon and its codon. Additionally, sedimentation studies (35) and functional properties (36) show that structural changes in tRNA synthetase affect aminoacylation (37), as do differing ratios in $\mathrm{Mg}^{++}$and ATP concentration levels. This can be partially explained by the suggestion that $\mathrm{Mg}^{++}$induces strong hydrogen bonding at the backbone thereby influencing the double helical regions to become more compact (38). Appropriately enough different aminoacyl synthetases apparently require different structural features in their cognate tRNAs for recognition (27). Replacement of $\mathrm{Mg}^{++}$by different polyamines such as putrescine, spermine, and spermidine is found to have little affect on aminoacylation (29). $\mathrm{Mn}^{++}$can also be substituted and when a comparison is made with the latter two polyamines, spermine has the stronger binding constant (39) partially attributed to the large size of the molecule in that it can bridge adjacent phosphates. This 
supports the contention that certain sites created by tertiary folding are responsible for cooperative binding (40). Also, looking at different alkaline salts (4l) it is found that tertiary structure is more stabilized by a decrease in cationic radius. However when various divalent ions are specifically observed as replacement ions for $\mathrm{Mg}^{++}$, it is noted that $\mathrm{Ca}^{++}$behaves quite similarly, indicating that due to their differing ionic radii but similar coordination properties, it is this coordination property rather than size of the ion that bears primary importance (20). This supports the idea that specific coordination sites are formed by the folded tRNA molecule. With the use of $\mathrm{Eu}^{+3}$, NMR studies have also yielded information concerning the location of different tRNA stems in the folded molecule (12) that $x$-ray diffraction more readily affords, though in the crystalline state. NMR assignments have also determined that certain resonances are sensitive to the tertiary structure (14) and furthermore that most of the stabilization of the secondary structure of the molecule exists already in smaller fragments which can be separated from an intact tRNA molecule. Shifts in resonances caused by neighboring residues can help describe the type of interactions involved in the formation of $t R N A$ through recombination of the smaller fragments.

Spectroscopic labelling can be used for the purpose of observing stacking interactions (42). Also the strongly 
fluorescent nucleotide residue commonly referred to as $Y$ has been used to estimate intramolecular distances on the basis of singlet-singlet energy transfer experiments (43). Triplet-triplet transfer and quenching offer information concerning anticodon stacking with the $\mathrm{Y}$ base at low temperature studies (44) showing that they are probably not stacked.

Modification of various bases gives insight into which bases are exposed and thus reactive. If a base is only slightly reactive or completely unreactive to a reagent specific for that particular base, information is provided as to the position of the base in the tertiary structure and possible intramolecular interactions. To this extent selective modification of cytidine, uridine, guanosine, and dihydrouridine residues have been studied (33).

In the same sense ethidium bromide binding has proved to be a useful probe of tRNA structure. Aminoacylation alters the kinetics of ethidium bromide, indicating that uncharged tRNA and aminoacylated tRNA thereby have different conformations (26). Temperature-jump relaxation methods and stopped-flow methods are used to look at these effects in the presence and absence of $\mathrm{Mg}^{++}$ (which could it turn be compared with similar polyamine replacement studies).

Activation enthalpies and entropies for recombination of fragments (45) and the melting of various sections of the intact molecule have given data indicating rate- 
limiting steps involved in the processes.

As for more specific changes involved in aminoacylation or intramolecular stacking and analogous bonding interactions, such is beyond the scope of this paper and would require an undue amount of space to detail.

\section{2. tRNA in Solution and as a Crystal}

An important consideration that arises especially in x-ray diffraction studies, which of course are undertaken on the crystalline form of tRNA ${ }^{\text {Phe, }}$, is whether or not the structure of $\mathrm{RRNA}^{\mathrm{Phe}}$ is the same in the crystal as it is in solution. It has generally been assumed that no crucial or significant conformational change takes place when the molecule crystallizes.

To this end a few studies have been performed $(15,16$, 46). Chen 16) has determined from the laser-excited Raman spectrum that the ordered backbone configuration is maintained in aqueous and solid (amorphous powder) tRNA, though the degree of hydration of the solid is rather low by comparison. More recently Chen 15) has shown that the spectra for aqueous and crystalline (orthorhombic and hexagonal) tRNA ${ }^{\text {Phe }}$ show the same characteristic Raman frequencies and intensities, indicating their structures to be the same. Furthermore the Raman spectra of crystals redissolved in their mother liquor are identical to that of native $t R_{N A}{ }^{\text {Phe }}$ in the same solution. Removal of magnesium ion however gives a substantially altered spectrum, 
showing the importance of the ion in stabilizing the tertiary structure of tRNA.

Rhodes (46), through platirum binding to tRNA ${ }^{\text {Phe }}$ in the crystalline state and solution, has presented results that suggest the anticodon loop adopts similar conformations both in solution and in the crystal.

A problem that obtains when doing crystallization studies of tRNA ${ }^{\text {Phe }}$ is the fact that organic solvents are used in the crystallization conditions. Ultraviolet and circular dichroic spectroscopic investigations have shown that denaturation of tRNA is caused by addition of organic solvents (22). A partial solution to this problem is to equilibrate the crystal with water to remove the organic solvent from the crystal lattice prior to crystallographic studies or else to crystallize the tRNA under conditions not using such solvents.

\section{Orthorhombic and Monoclinic Crystal Form Comparison}

A question of interest nearly tantamount to that of the previous section is concerned with whether different crystal forms have the same structure with regard to the tRNA molecule. Along these lines the orthorhombic and monoclinic crystals have been looked at to some extent. The two crystal forms are essentially the same except for five particular residue position and base and sugar conformations as noted by Sussman (47). Quigley (4) cites 
the only disparity between the orthorhombic and monoclinic lattices as being in the conformation near the $3^{\prime}$ end of the polynucleotide chain. Another interesting observation is that both lattices also appear to contain a guanine-uracil base pair held together by two hydrogen bonds (4).

By changing the packing of the tRNA ${ }^{\text {Phe }}$ molecule from a head-to-head and tail-to-tail arrangement which has been used to describe the orthorhombic lattice to a headto-tail packing arrangement, the monoclinic unit cell can be generated (6), further lending credence to the belief that there is no difference in the density maps and three dimensional representations of $t \mathrm{RNA}^{\mathrm{Phe}}$.

\section{Oriented Film Spectra of Nucleic Acids}

Since crystals of DNA are not readily available, the method of drawing fibres and films of the nucleic acid has been utilized to study $(48,49,50,51,52)$ solid DNA in a crystal-like form. Preparation of the films has been well described and necessitates developing some familiarity with the technique involved in order to achieve uniform films. Essentially the oriented films are such that the major portion of DNA is aligned along its helical axis so that light can be subsequently polarized both parallel and perpendicular to the helical axis. The parallel polarization is here taken to correspond to the $z$ polarization while the perpendicular polari- 
zation is taken to correspond to both the $\mathrm{x}$ and $\mathrm{y}$ directions, which are assumed to be equivalent because of the helical form of the molecule.

In a sample of well-oriented DNA molecules, the purine and pyrimidine bases can be considered as having their conjugated rings approximately perpendicular to the long axis of the helix. Explanation of the electronic transitions has been ably presented (52) and has set a basis for the theoretical work.

DNA is considered to exist primarily in two different conformations, generally called the $\mathrm{A}$ and the $\mathrm{B}$ forms. Studies have been carried out to try to elucidate which form exists under certain conditions; that is whether the A form or the B form predominates as the relative humidity is changed in films. The salt concentration in films has also been observed to influence the particular conformation. This has been looked at in terms of total absorption and the dichroic ratio and still remains somewhat unresolved $(50)$. It should be pointed out that this problem is not something this paper will attempt to address however. Nonetheless it is of some concern to note that the total UV absorption of oriented films does vary as a function of relative humidity. Thus this is of particular concern in discussing structural comparisons or differences.

Both UV absorption spectra and Circular Dichroism 
spectra of various types of DNA have been published and discussed. This study will concern itself with only the absorption spectra since it is from these spectra that extinction coefficients of DNA can be derived for the $\mathrm{x}, \mathrm{y}$, and $\mathrm{z}$ polarizations. Furthermore it is only the oriented film spectra that are of interest and not the unoriented spectra which indeed have also been published. An unoriented film at a high relative humidity is essentially the same as a solution of DNA as the spectra of these samples are identical (51).

Absorption spectra of Calf Thymus DNA films have been published (48) and are presented in figure 3.' Due to the instrumentation used, the wavelength region measured only goes down to $240 \mathrm{~nm}$. The results of these spectra are interpreted (48) as indicating that the purine and pyrimidine bases are arranged so that on the average they lie at an angle slightly less than normal to the molecular axis and that this angle decreases with increasing humidity.

Absorption spectra of T2 bacteriophage DNA have also been published (52), though in considerably more detail. First of all the spectra were taken over a wider wavelength region $(215 \mathrm{~nm}$ to $300 \mathrm{~nm})$. Additionally a greater variety of relative humidities were ob served under experimental conditions. Finally it was noted that the dichroic ratio differed at the same wavelength as a 


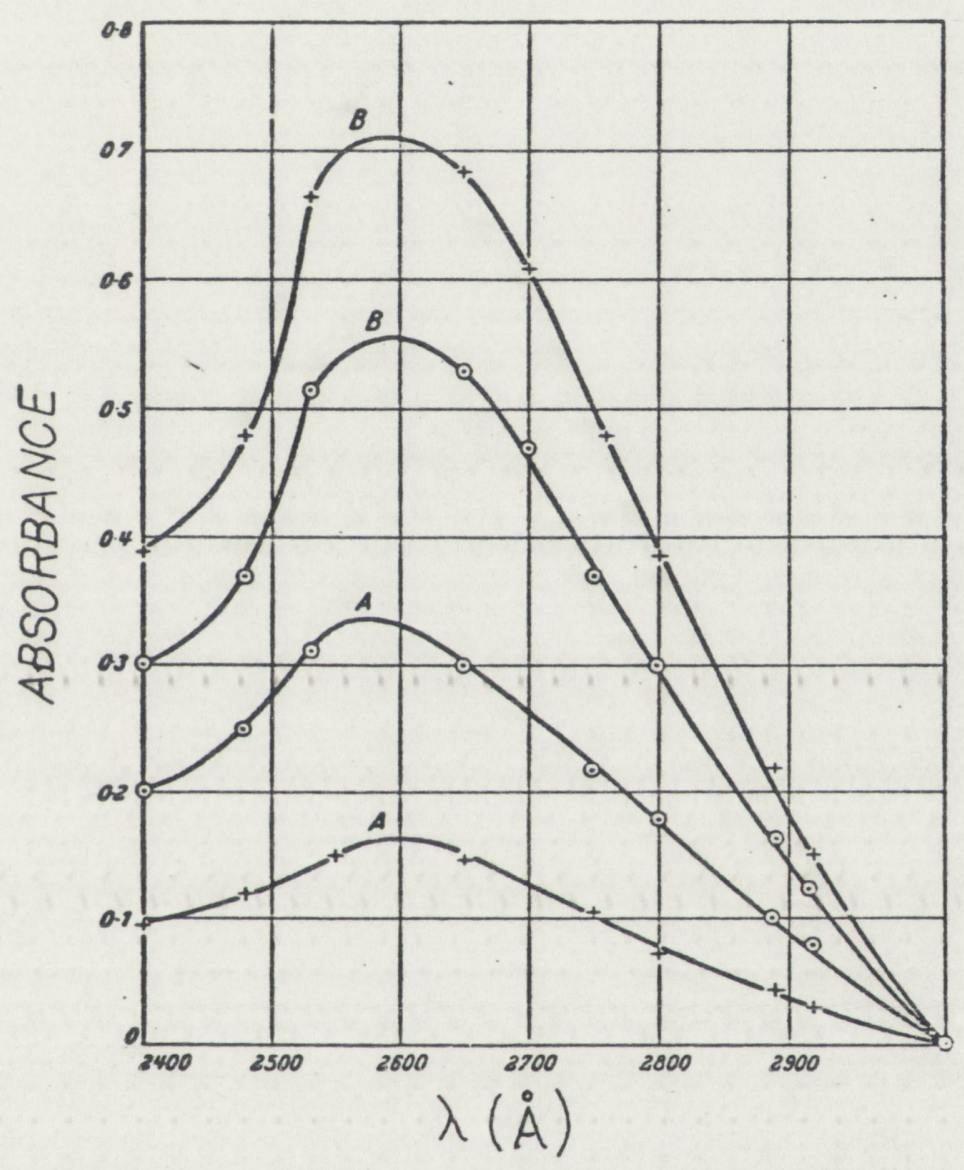

Figure 3. Ultraviolet absorption curves of calf thymus DNA under different conditions of humidity: (A) Electric vector parallel and (B) Electric vector perpendicular to direction of shear. (+) at $90 \%$ humidity and $(\theta)$ at $60 \%$ humidity. 
function of whether a particular relative humidity was attained by either increasing or decreasing that of the same previously measured film. Figures 4-6 (52) show the T2 spectra both obtained by increasing the humidity and decreasing the humidity, plus the trend of the dichroic ratio at $260 \mathrm{~nm}$.

It is difficult to determine from the dichroic ratio measurements of the DNA film whether the A form or the $\mathrm{B}$ form exists. However through $\mathrm{x}$-ray diffraction experiments it has been determined (50) that the B conformation exists at high relative humidities. Finally, perhaps the subject of greatest concern in the literature is possible reasons for changes in the dichroic ratio. There has been much discussion regarding $n-\pi$ * transitions (polarized perpendicular to the plane of the purine and pyrimidine bases) and $\pi-\pi$ * transitions (polarized in the plane of the bases) as being implicated for certain absorption bands in the ultraviolet region under particular experimental conditions. This presently seems to remain in question to some extent.

\section{Flow Dichroism}

Flow dichroism is a method for biomolecular study essentially equivalent to electric dichroism. Both methods could be generally termed linear dichroism since the sample under study is usually oriented along its 


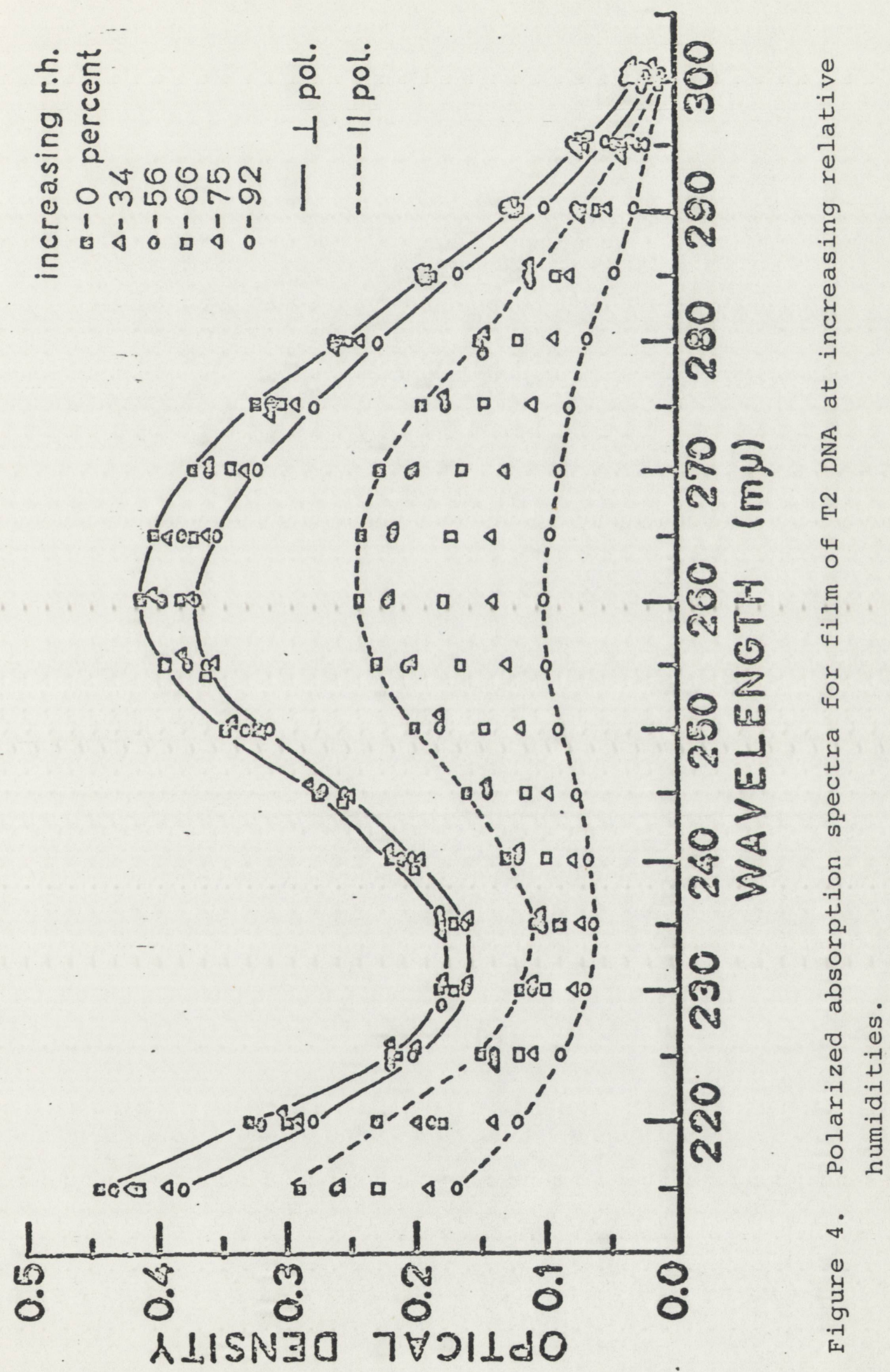




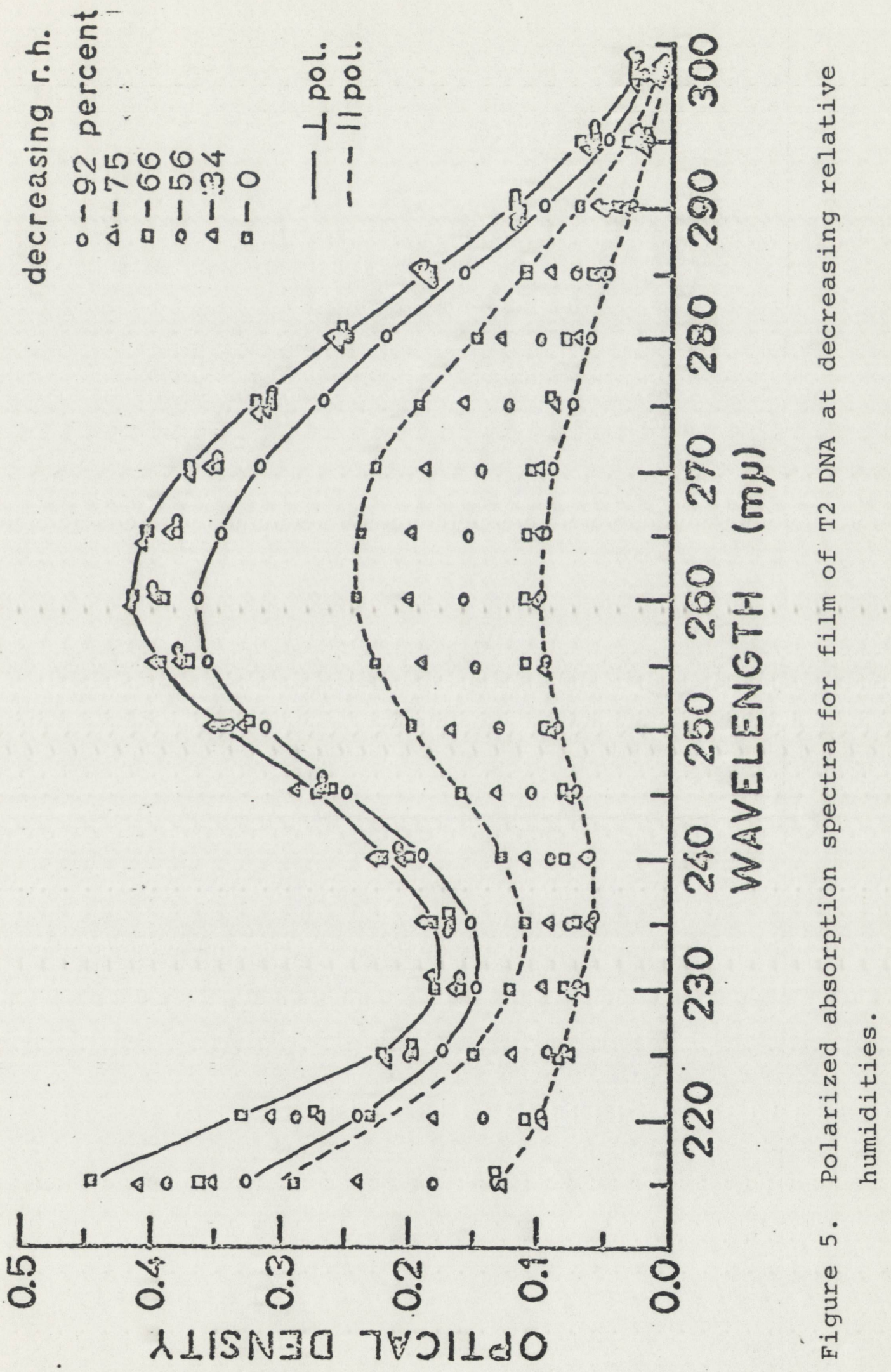




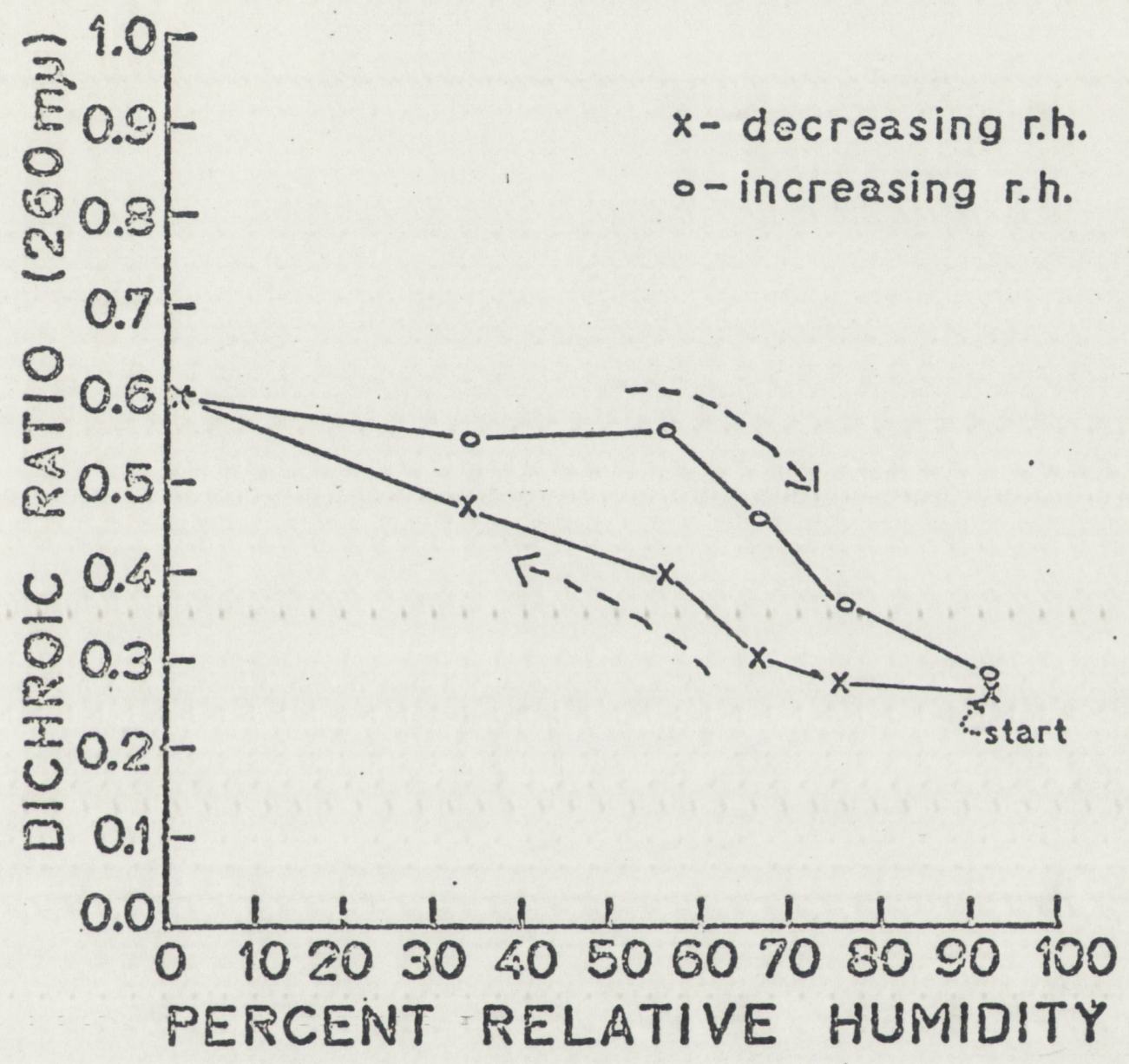

Figure 6. Humidity history of film. 
long molecular axis. In flow dichroism the molecules are oriented in a solution under the shear of constant flow. The shear rate that determines the average orientation of a sample is a function of the molecular dimensions and will determine the extent of dichroism observed. The dichroism thus observed is the difference between the absorption of light polarized parallel and that polarized perpendicular to the molecular axis of the molecule or flow lines of the solution.

The method of flow dichroism has the advantage of not being restricted by ionic strengths of a solution. The techniques and the apparatus involved have been described in the literature $(53,54,50,55)$. Both forms of linear dichroism are capable of denaturing samples such as DNA. While electric dichroism can heat denature the DNA, flow dichroism can denature DNA through a high rate of shear-which however is normally not a problem since those rates are seldom attainable (55). Additionally just as in electric dichroism when saturation field voltage has not been reached and the sample in solution has not been completely oriented on the average, so the same occurs in flow dichroism when "infinite shear rate" has not been attained. In this pre-saturation condition the planar rings on the DNA which are responsible for absorption are at some average angle to the flow lines. This angle will be less than perpendicular 
or the maximum obtainable angle and hence the dichroism which is partially a function of this angle will be less than that observed at saturation or infinite shear. Thus flow dichroism is a function of flow rate. Gray and Rubenstein (50) have published flow dichroism for T2 DNA. Polarizations parallel and perpendicular to the flow at zero velocity gradient yield identical spectra. However when a velocity gradient of $3040 \mathrm{sec}^{-1}$ is used, the spectra for the two polarizations differ considerably, indicative of the anisotropy of the molecule. The perpendicular polarization absorbs much more than the parallel, an observation which is reasonable since the former polarization is in the plane of the base rings corresponding to a $\pi-\pi{ }^{*}$ transition.

One difficulty that is involved with flow dichroism is that of orientation factors. The matter of both internal and external orientation factors has been discussed in detail (55). In the case of a flexible molecule, as in DNA, the macromolecular conformation and hence the orientation of the base planes changes as a function of the flow gradient, so that the orientation factors become inseparable. It is noteworthy however that under a velocity gradient DNA behaves as a rigid rod and at very high velocities is somewhat stretched (55). 
6. Comparisons Between Flow Dichroism Spectra and Oriented Film Absorption Spectra

The essential difference between flow dichroism spectra and film absorption spectra develops because the biomolecule is observed under two disparate conditions. In the former the molecule is in solution, while in the latter it is an oriented solid, somewhat analogous to being in a crystal lattice since the molecules are in some regular array.

For DNA the wavelength dependence of the dichroic ratio is the same for both film and solution, an observation which suggests no contribution from form dichroism (50). This indicates a very similar structure for DNA in the two environments, though no particular correlation between solution and film structure of DNA was attempted. One fact that was determined from these studies using T2 and T5 DNA, was that the dichroic ratio is insensitive to base plane tilt and that the dichroic ratio can not be used to distinguish between the $\mathrm{A}$ and $\mathrm{B}$ configurations of DNA (50). X-ray diffraction has proven however to be a useful tool in this matter. Finally it should be noted that the oriented film absorption spectra and flow dichroism spectra look very much alike, both giving identical minima and maxima. 


\section{Oriented Film Spectra of Polynucleotides}

Just as oriented films of DNA have been prepared and their ultraviolet absorption spectra published, so have the polynucleotides polycytidylic acid (56) and polydeoxyadenylic-deoxythymidylic acid (poly-(dAT)) (57). Similar to DNA, polycytidylic acid forms a helix (56). The cytosine residues are oriented perpendicular to the helical axis as is typical of the bases in DNA. As is apparent from the spectra for polycytidylic acid, the absorption of the light vector in the two orthogonal directions is substantially different. Unfortunately Rich and Kasha only published the oriented film spectra at $50 \%$ relative humidity without doing so at any other relative humidities in order to observe any possible changes.

The oriented film spectra of poly-(dAT) was likewise unfortunately recorded at only $60 \%$ relative humidity. The difference in the two polarizations is not quite as striking as in polycytidylic acid. Fortunately, again as with polycytidylic acid, poly-(dAT) is a duplex

whose dimensions are those of double stranded DNA (58). Thus because of the helical form for the two polynucleotides discussed above it should be possible to record the linear dichroism spectra for solutions of the same. Their rigid structures should insure that 


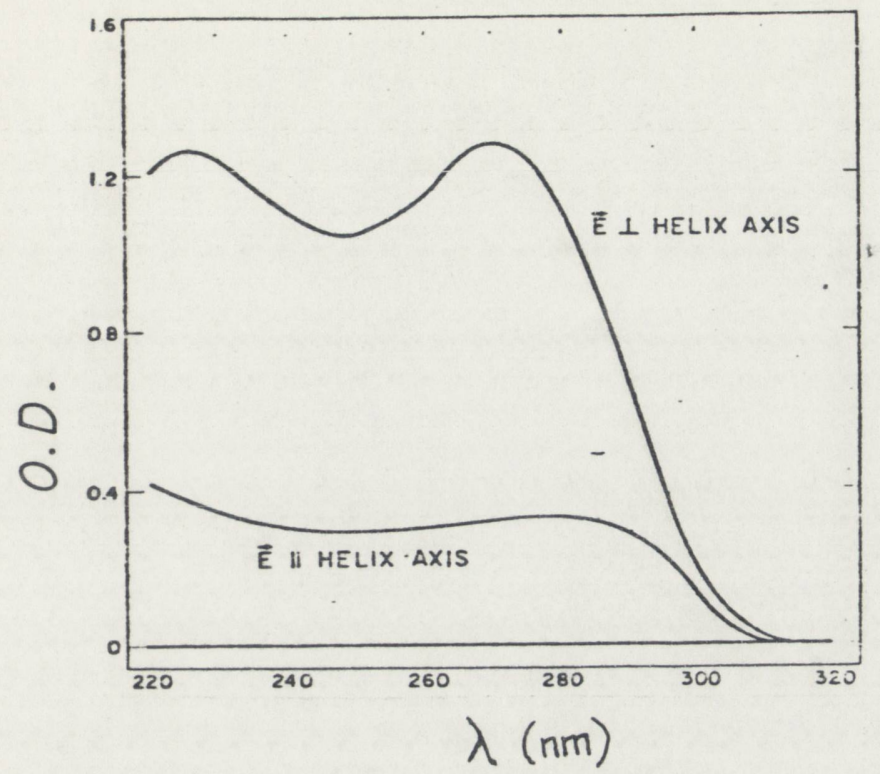

Figure 7. Ultraviolet absorption spectra of oriented film of polycytidylic acid at $50 \%$ relative humidity.

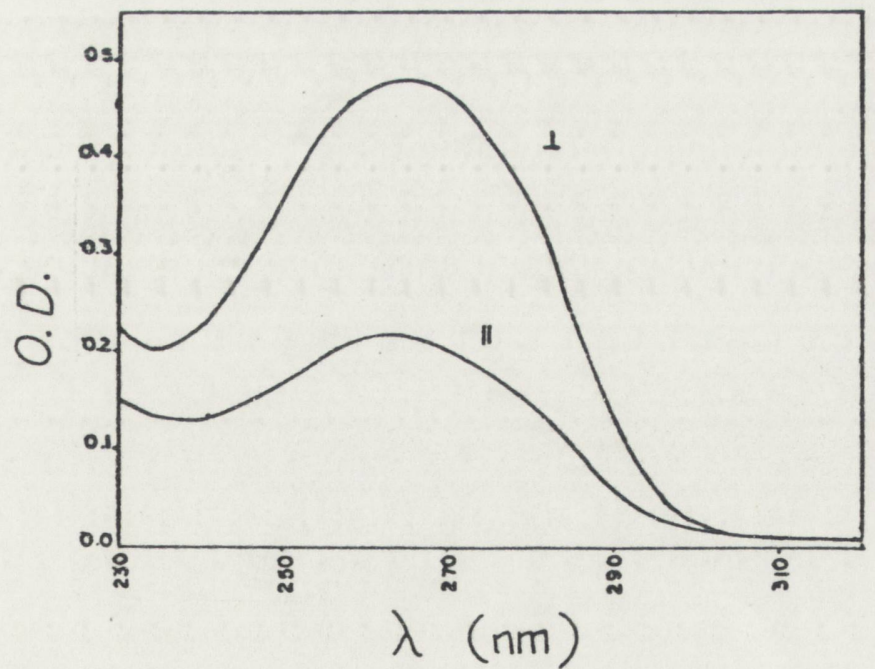

Figure 8. Ultraviolet absorption spectra of oriented film of poly (d-AT) at $60 \%$ relative humidity. 
they will be oriented by an electric field. The double stranded helix of polyadenylic + polyuridylic acid (poly $A+U$ ) plus any other polynucleotides with similar structure should behave essentially the same. Therefore any conclusions drawn from polycytidylic acid and poly(dAT) should be applicable to other polynucleotides having helical structures. 
With the purpose of furthering the knowledge of whether the structure of tRNA ${ }^{\text {Phe }}$ is the same in the crystal as it is in solution, the following experiment has been conceived. A comparison of the polarized ultraviolet absorption spectra of the $\mathrm{RNA}^{\mathrm{Phe}}$ in three mutually orthogonal directions is made with the electric dichroic spectra of the tRNA ${ }^{\text {Phe }}$ using an instrument constructed in our laboratory. (To facilitate obtaining the indicated absorption spectra so that the crystal itself does not have to be rotated through two orthogonal directions a rotatable polarizer is used. This expedites an otherwise physically difficult problem.)

The information that the spectra afford are the extinction coefficients in the $x, y$, and $z$ directions (from the polarized absorption spectra) and those in the direction parallel and perpendicular to the applied electric field (from the solution spectra). That is, from ultraviolet solution absorption spectra one can obtain the parameter $\varepsilon_{0}$ and from the electric dichroic spectra $\Delta \varepsilon$ is obtained, where

$$
\varepsilon_{0}=\frac{\varepsilon_{\mathbb{l}}+2 \varepsilon_{\perp}}{3} \quad \Delta \varepsilon=\varepsilon_{\mathbb{l}}-\varepsilon_{\perp}
$$

With these two equations one can simply solve for the two unknowns, $\varepsilon_{\mathbb{\|}}$ and $\varepsilon_{\perp}$, at each wavelength measured. The problem then that exists is deciding whether or not $\varepsilon_{x}$, $\varepsilon_{y^{\prime}}$ and $\varepsilon_{z}$ are related to $\varepsilon_{\|}$and $\varepsilon_{\perp}$. If they are related 

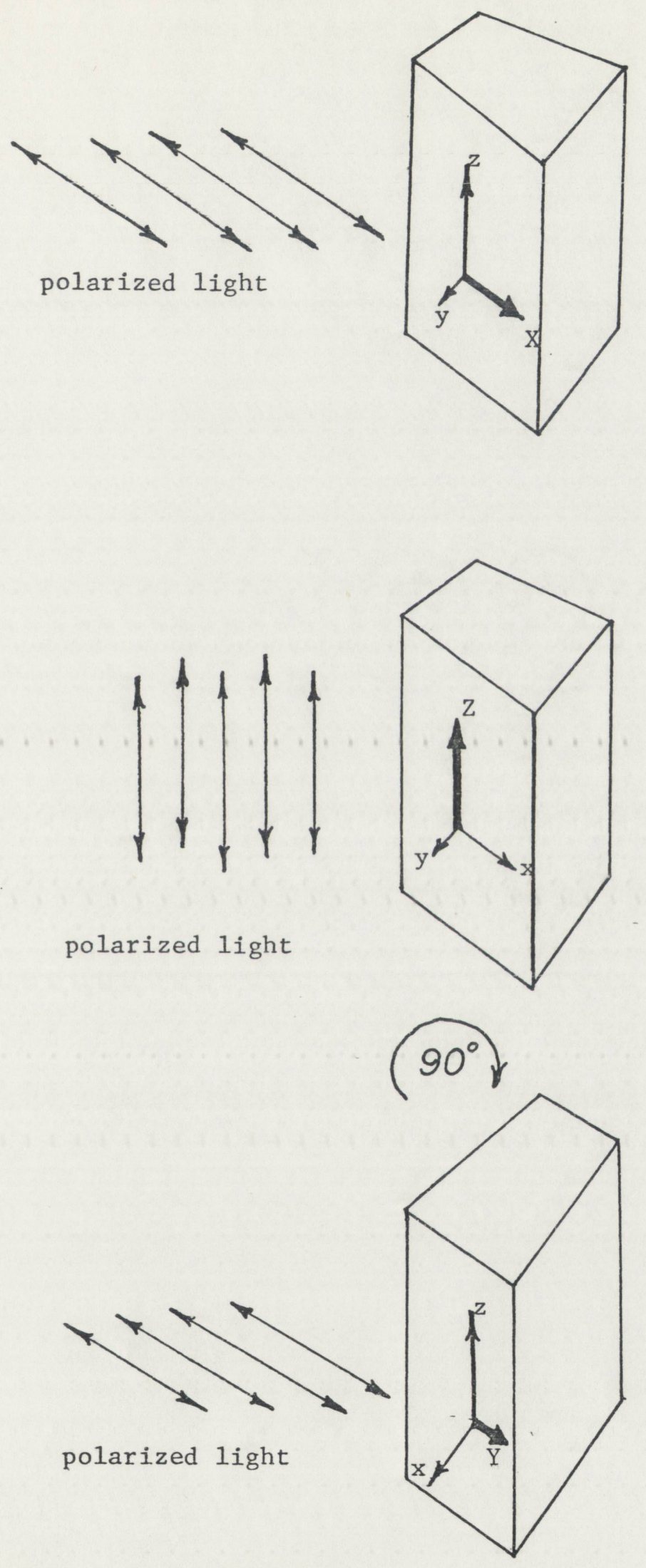

Figure 9. $\mathrm{X}, \mathrm{Y}$, and $\mathrm{Z}$ polarizations in a crystal. 
then there must be a rotation matrix that will rotate the one into the other indicating that the crystal structure and solution structure of $\mathrm{tRNA}^{\mathrm{Phe}}$ are identical and that the solution spectra can be considered as a linear combination of the crystal spectra. The two sets of data taken at various wavelengths can then be equated as follows :

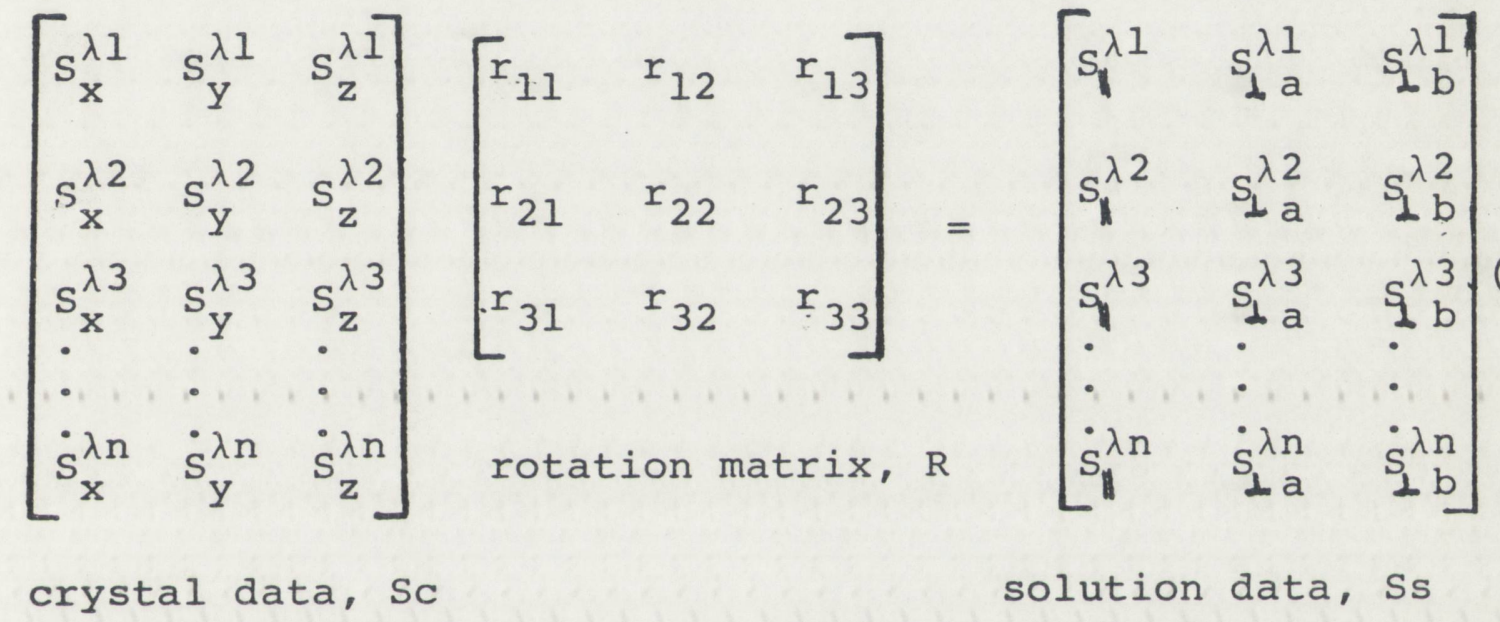

Furthermore, $\quad 1 / 2\left(\mathrm{~s}_{a}^{\lambda}+\mathrm{s}^{\lambda}{ }_{\mathrm{b}}\right)=\mathrm{s}^{\lambda}$, where $\mathrm{s}_{i}$ is orthogonal to $S_{j}$. That is, $S$ is a linear combination of $s_{i}$ and $s_{j}$, so we can write:

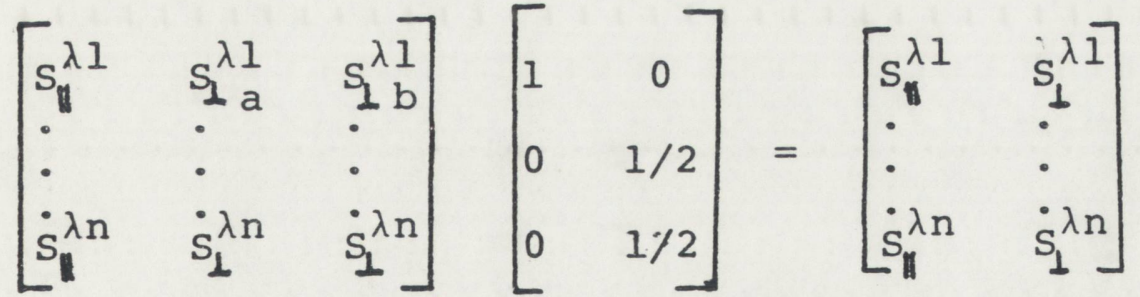

$$
\begin{aligned}
& \text { A Electric dichroism data } \\
& \mathrm{S}_{\mathrm{ED}}
\end{aligned}
$$

The above equations (1) and (2) can be more simply expressed respectively as:

$$
\begin{aligned}
& \mathrm{S}_{\mathrm{C}} \times \mathrm{R}=\mathrm{S}_{\mathrm{S}} \quad \text { and } \\
& n \times 3 \quad 3 \times 3 \quad n \times 3 \\
& \mathrm{~S}_{\mathrm{S}} \times \mathrm{A}=\mathrm{S}_{\mathrm{ED}} \\
& \mathrm{n} \times 3 \quad 3 \times 2 \quad \mathrm{n} \times 2
\end{aligned}
$$


Where the number below the matrix representation is an indication of the dimension of that particular matrix, with $n$, the number of rows, representing the number of different wavelengths at which the data is recorded.

Then, using matrix multiplication we can derive an equation which will equate the crystal spectra data (through the rotation matrix) to the electric dichroic data which was our original intent.

Multiplying both sides of equation (1) by matrix A we obtain:

$$
\begin{aligned}
& S_{C} \times R \times A=S_{S} \times A \quad \text { which also equals } S_{E D} \\
& n \times 3 \quad 3 \times 3 \quad 3 \times 2 \quad n \times 3 \quad 3 \times 2
\end{aligned}
$$

$$
\text { or } \begin{array}{r}
S_{C} \times R^{*}=S_{E D} \\
n \times 3 \quad 3 \times 2 \quad n \times 2
\end{array}
$$

where $R^{*}=R \times A$

that is,

$$
R^{*}=\left[\begin{array}{lll}
r_{11} & r_{12} & r_{13} \\
r_{21} & r_{22} & r_{23} \\
r_{31} & r_{32} & r_{33}
\end{array}\right]\left[\begin{array}{ll}
\mid l & 0 \\
0 & 1 / 2 \\
0 & 1 / 2
\end{array}\right]=\left[\begin{array}{ll}
r_{11} & \left(r_{12}+r_{13}\right) / 2 \\
r_{21} & \left(r_{22}+r_{23}\right) / 2 \\
r_{31} & \left(r_{32}+r_{33}\right) / 2
\end{array}\right]=\left[\begin{array}{cc}
r_{11}^{*} & r_{12}^{*} \\
r_{21}^{*} & r_{22}^{*} \\
r_{31}^{*} & r_{32}^{*}
\end{array}\right]
$$

Thus as far as equation (3) is concerned, the only unknown is the rotation matrix, $R^{*}$, since $S_{C}$ and $S_{E D}$ are extracted from the crystal and solution spectra, respectively. Now however we need to calculate $R^{*}$. By left multiplying 
both sides of equation (3) by the transpose of $S_{C}$ we get:

$$
\begin{aligned}
& S_{C}^{t} \times S_{C} \times R^{*}=S_{C}^{t} \times S_{E D} \\
& 3 \times n \quad n \times 3 \quad 3 \times 2 \quad 3 \times n \quad n \times 2
\end{aligned}
$$

We now have the square matrix $S_{C}^{t} \times S_{C}$ on the left side of equation (4), which when multiplied by its inverse matrix will give a unitary matrix.

$$
\begin{aligned}
& \left(S_{C}^{t} S_{C}\right)^{-1} \times\left(S_{C}^{t} S_{C}\right) \times R^{*}=R^{*}=\left(S_{C}^{t} S_{C}\right)^{-1} \times S_{C}^{t} \times S_{E D} \\
& \begin{array}{lllllll}
3 \times 3 & 3 \times 3 & 3 \times 2 & 3 \times 2 & 3 \times 3 & 3 \times n & n \times 2
\end{array}
\end{aligned}
$$

and we obtain a least squares solution for $\mathrm{R}^{*}$ which can be solved by computer.

The analytical form of $\mathrm{R}^{*}$ can be obtained by Euler's rotation in 3 dimensions described by 3 matrices (68) which are then multiplied together to give (7).

$$
\begin{gathered}
R_{Z}(\gamma)=\left[\begin{array}{ccc}
\cos \gamma & \sin \gamma & 0 \\
-\sin \gamma & \cos \gamma & 0 \\
0 & 0 & 1
\end{array}\right] ; R_{Y}(\beta)=\left[\begin{array}{ccc}
\cos \beta & 0 & -\sin \beta \\
0 & 1 & 0 \\
\sin \beta & 0 & \cos \beta
\end{array}\right] \\
R_{z}(\alpha)=\left[\begin{array}{ccc}
\cos \alpha & \sin \alpha & 0 \\
-\sin \alpha & \cos \alpha & 0 \\
0 & 0 & 1
\end{array}\right]
\end{gathered}
$$

such that $R(\alpha, \beta, \gamma)=R_{z}(\alpha) R_{y}(\beta) R_{z}(\gamma)$, which describes rotation about the indicated axis through the indicated angle. 
$\Xi$

$\Phi$

9

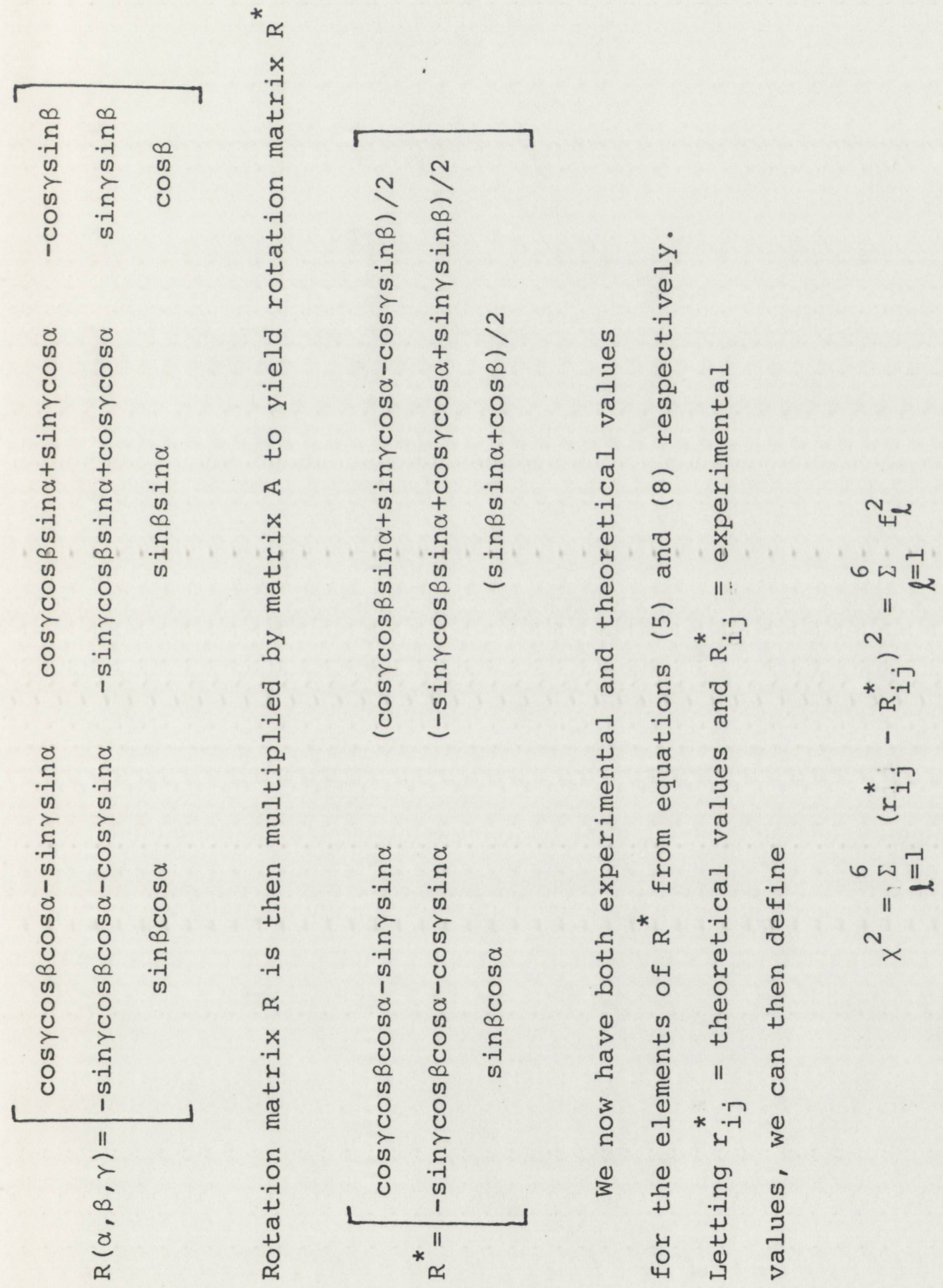


where ideally $x^{2}=0$ and generally we want to find the angles $\alpha, \beta$, and $\gamma$ which minimize $x^{2}$ showing that the theoretical and experimental values are the same or similar. In equation (9)

$$
\begin{array}{ll}
f_{1}=r_{11}^{*}-R_{11}^{*} & f_{2}=r_{21}^{*}-R_{21}^{*} \\
f_{3}=r_{31}^{*}-R_{31}^{*} & f_{4}=r_{12}^{*}-R_{12}^{*} \\
f_{5}=r_{22}^{*}-R_{22}^{*} & f_{6}=r_{32}^{*}-R_{32}^{*}
\end{array}
$$

and in order to minimize $\chi^{2}$, we must take its derivative with respect to each of the three angles, ie.,

$$
\frac{\partial x^{2}}{\partial \alpha}=0, \quad \frac{\partial x^{2}}{\partial \beta}=0, \quad \text { and } \quad \frac{\partial x^{2}}{\partial \gamma}=0
$$

or

$$
\frac{\partial \chi^{2}}{\partial \alpha}=\frac{\partial \sum_{l} f_{l}^{2}}{\partial \alpha}=\sum_{l} \frac{\partial f_{l}^{2}}{\partial \alpha}=\sum_{l} 2 f_{l} \frac{d f_{l}}{d \alpha}=0
$$

likewise

$$
\frac{\partial \chi^{2}}{\partial B}=\sum_{l} 2 f \frac{d f}{d \beta}=0 \quad \text { and } \quad \frac{\partial \chi^{2}}{\partial \gamma}=\sum_{l} 2 f \frac{d f}{d \gamma} l=0
$$

The elements of the matrix containing the derivatives of the rotational matrix are as follows. (In the section describing the computer computations, these will comprise the elements of JACOB): 


$$
\begin{aligned}
& \frac{\partial f}{\partial \alpha} l=-\cos \gamma \cos \beta \sin \alpha-\sin \gamma \cos \alpha \\
& \frac{\partial f}{\partial \beta} I=-\cos \gamma \sin \beta \cos \alpha \\
& \frac{\partial f}{\partial \gamma} l=-\sin \gamma \cos \beta \cos \alpha-\cos \gamma \sin \alpha \\
& \frac{\partial f}{\partial \alpha} 2=\sin \gamma \cos \beta \sin \alpha-\cos \gamma \cos \alpha \\
& \frac{\partial f}{\partial \beta} 2=\sin \gamma \sin \beta \cos \alpha \\
& \frac{\partial f}{\partial \gamma} 2=-\cos \gamma \cos \beta \cos \alpha+\sin \gamma \sin \alpha \\
& \frac{\partial f}{\partial \alpha} 3=-\sin \beta \sin \alpha \\
& \frac{\partial f}{\partial \beta} 3=\cos \beta \cos \alpha \\
& \frac{\partial f}{\partial \gamma} 3=0 \\
& \frac{\partial f}{\partial \alpha} 4=1 / 2(\cos \gamma \cos \beta \cos \alpha-\sin \gamma \sin \alpha) \\
& \frac{\partial f}{\partial \beta} 4=1 / 2(-\cos \gamma \sin \beta \sin \alpha-\cos \gamma \cos \beta) \\
& \frac{\partial f}{\partial \gamma} 4=1 / 2(-\sin \gamma \cos \beta \sin \alpha+\cos \gamma \cos \alpha+\sin \gamma \sin \beta) \\
& \frac{\partial f}{\partial \alpha} 5=1 / 2(-\sin \gamma \cos \beta \cos \alpha-\cos \gamma \sin \alpha) \\
& \frac{\partial f}{\partial \beta} 5=1 / 2(\sin \gamma \sin \beta \sin \alpha+\sin \gamma \cos \beta) \\
& \frac{\partial f}{\partial \gamma} 5=1 / 2(-\cos \gamma \cos \beta \sin \alpha-\sin \gamma \cos \alpha+\cos \gamma \sin \beta) \\
& \frac{\partial f}{\partial \alpha} 6=1 / 2(\sin \beta \cos \alpha) \\
& \frac{\partial f}{\partial \beta} \sigma=1 / 2(\cos \beta \sin \alpha-\sin \beta) \\
& \frac{\partial f}{\partial \gamma} \sigma=0
\end{aligned}
$$


Thus by minimizing $\chi^{2}$ we can obtain the angles $\alpha$, $\beta$, and $\gamma$ which will define the Eulerian rotation comparing the crystal structure to the solution structure. 


\section{COMPUTER TREATMENT}

First of all in order to determine the elements of the experimental rotation matrix and then the Eulerian angles of the theoretical rotation matrix, a computer program has been written which will compute these values. The program is given in the appendix and described below.

The program reads in the crystal and solution data which have been collected. Subroutine LINIT is then called which solves matrix equation (5) so that the experimental rotation matrix can be obtained. This matrix together with an initial guess at the three Eulerian angles provides the starting point for determining the actual values of the Eulerian angles.

Essentially a surface in four dimensional space is generated on which we are looking for a minimum, corresponding to the minimum value of $\chi^{2}$ (which is defined as SUMSQ in the program) yielding the appropriate values for the angles. The first guess is used for deciding in which direction to proceed. Subroutine SEARCH is called for this purpose which directly calls subroutine EVAL to calculate the six component values of $x^{2}$ (ie., $f(1 \rightarrow 6)$ as in equation 9) from which SUMSQ is computed as are subsequently the eighteen elements of JACOB (as listed on page 31). Finally EVAL determines the gradient of the surface, GRAD, at this initial guess before returning control to SEARCH. 
In order to improve our initial guess, it is now desired to move in the direction of steepest descent on the $x^{2}$ surface. This movement, if indeed it is in the correct direction, will be evidenced by a diminution in the value of SUMSQ. Moreover, once we have begun descending down the $x^{2}$ surface, the size of the STEP taken to reach the minimum on the surface will be augmented by a factor of four for the purpose of attaining that point more quickly.

However if it occurs that through this larger STEP the minimum is overshot, that is, rather than GA decreasing, it begins to increase and we are on the upswing of the surface, a parabola is fitted between the last two points, STEP is cut back by a fourth, and SEARCH again looks for a minimum.

When an improvement of the current guess is desired, SEARCH calls FETCH which initiates an iterative improvement step. FETCH calls GENLS which accepts the nonsquare $6 \times 3$ matrix, JACOB, under the name ARRAY and a vector of constants, CVECT (which is the $x^{2}$ matrix in EVAL). GENLS transforms ARRAY into the square matrix $A A$, having the same general properties. SVECT is the direction vector (called $\mathrm{S}$ in SEARCH) which indicates the direction to move on the surface and is a vector by which ARRAY is multiplied. At the same time that ARRAY is transformed to $A$, by multiplying by its transpose, CVECT is transformed to AC. Thus we have the following: 


$$
\text { ARRAY } \times \text { SVECT }=\text { CVECT }
$$

$6 \times 3 \quad 3 \times 1 \quad 6 \times 1$

multiplying by the transpose of ARRAY:

$$
\begin{array}{ccccc}
\text { ARRAY }^{t} & \text { x ARRAY } & \text { x SVECT } & \text { ARRAY } & \text { AR } \\
3 \times 6 & 6 \times 3 & 3 \times 1 & 3 \times 6 & 6 \times 1
\end{array}
$$

to obtain:

$$
\begin{aligned}
& A A \times \text { SVECT }= \\
& 3 \times 3 \quad 3 \times 1 \quad 3 \times 1
\end{aligned}
$$

GENLS calls subroutine SYMSOL, which accepts AA (working with it as matrix $A$ ) and finds the solution vector $S, \bar{S}$, of the square matrix, operating on the square root method of Banachiewicz. Essentially $\overline{\mathrm{S}}$ is a direction to move (in three dimensions) in order to obtain a better solution, thus $\bar{S}$ is not the solution in itself.

Once $\bar{S}$ (which has the name SVECT in subroutine GENLS and the name $\mathrm{V}$ in subroutine SYMSOL) is calculated, control returns to FETCH which then undertakes examination of this vector. If it happens that $A A$ was nearly singular then $\bar{S}$ is not a useful direction vector and GRAD is substituted. Control is then returned to SEARCH. A near singular matrix can result from experimental error or when one element of $\bar{S}$ is much closer to the true solution than the other two elements. A singular matrix is considered by FETCH to be one in which the largest element of $\overline{\mathrm{s}}$ is greater than $10^{5}$ times the smallest element. 
If $\bar{S}$ is nearly singular FETCH substitutes the negative gradient of SUMSQ (GRAD) which should point in the direction of steepest descent, toward minimum $x^{2}$. Using this value of GRAD accomplishes the same thing, however it provides a slower convergence. Finally having checked $\overline{\mathrm{S}}$, control is returned again to SEARCH.

In order to determine what kind of progress is being made each time the program loops through the system, newly computed values are compared to previous values of particular variables. To this end $\overline{\mathrm{S}}$ is compared to its former value, now called SIGMA, just as SUMSQ is also similarly scrutinized, as described earlier. In each case the new value should be smaller than the old value, indicating that the calculations are converging on the minimum of the surface. The program itself has frequent comment statements in many of the subroutines detailing the sequence of computation involved.

The variable MAX is a count on how many times the iteration or cycling through the series of step is done. In MAIN, MAX is set equal to some number so as to limit the number of loops.

Execution of the program is terminated when the value of SUMSQ has reached the point where it undergoes very little change, that is when the ratio of the new value of SUMSQ to the old value (or FB/OLDF) is greater than 0.999999, evidencing that the minimum has been reached. The percent error in the calculation is 
reported as the absolute value of the summation of the difference between the theoretical and experimental values of the rotational matrix divided by the experimental values,

$$
\text { ie., } \% \text { ERROR }=\left[\sum_{i=1}^{6}\left(r_{i}-R_{i}\right) / R_{i}\right] \times 100 \%
$$

The minimum SuMSQ is then printed, as are the gradients at that point. All these are numbers which should be rather small and essentially zero, if indeed a rotation matrix exists which equates the crystal spectra data to the solution spectra data. Returning to MAIN, the program finally prints the three angles in degrees, which are the unknown vectors.

The function DOT which is called at numerous points through the program is used to find the dot or scalar product of vectors. Subroutine SYMSOL can be replaced with LINIT, which is a subroutine used earlier and which itself calls several other subroutines and does the same thing as SYMSOL, though a little more accurately and also performs a check on matrix singularity.

The program described above unfortunately could not be used when work on tRNA ceased. When oriented film absorption spectra are used rather than crystal data, the rotation matrix takes on a much simpler form. Since the dipole moment axis is the same as the helical axis it is 
valid to consider rotations in the $\mathrm{x}$ and $\mathrm{z}$ directions as the only rotations existing. That is, the helical axis is symmetrical when there are no unusual bases along it. Thus rotation about the helical axis does not change anything and this angle then is considered fixed. By moving the $x$ and $z$ axes through angle theta we are essentially rotating both these axes.

In order to account for this more simplified rotation only one rotational matrix is necessary which we have defined to be as follows:

$$
R_{\theta}=\left[\begin{array}{rr}
\cos \theta & \sin \theta \\
-\sin \theta & \cos \theta
\end{array}\right]
$$

This is a simpler Euler rotation, necessary only for rotation in one dimension.

The main program which does this calculation, a degeneration of the previous and more extensive program given in Appendix $A$, is given in Appendix B. Only the main program is included-which also necessitates the subroutine LINIT (and its ancillary subroutines). However this matrix method proved to be too sensitive to any noise in solving for the rotational matrix, therefore a linear programming method was resorted to for solving the problem.

An excellent subroutine which was available for this purpose is called SIMPLX. Its usage for this particular problem will herein be described. A rather unique formulation 
resulted in order to make greatest utilization of SIMPLX: it was iterated to work as a nonlinear program- an unusual thing for a linear program to do.

The main program first reads in the electric dichroism and solution absorption data, $\Delta \varepsilon$ and $\varepsilon_{0}$, respectively plus the film absorption data. Perpendicular and parallel solution absorption values are calculated and then normalized as are the film data. The data are normalized by summing the squares of each value resulting in a vector value. The initial matrix mathematics take on the following form (and names) in the program: $S_{C} \cdot R=S_{E D}$ (where $S_{C}$ is the film data, $R$ is the rotation matrix as given above, and $\mathrm{S}_{\mathrm{ED}}$ is the solution data) ...multiplying each side by the transpose of $\mathrm{S}_{\mathrm{C}}$

$$
\begin{array}{rrrrr}
s_{C}^{t} \cdot & s_{C} & R= & s_{C}^{t} & s_{E D} \\
2 x n & n \times 2 & 2 \times 2 & 2 \times n & n \times 2
\end{array}
$$

or

$$
\begin{aligned}
& X I N \cdot R=S S \\
& 2 \times 2 \quad 2 \times 2 \quad 2 \times 2
\end{aligned}
$$

Now we call upon SIMPLX, after first describing the objective function which we want to minimize plus any constraints deemed necessary that the function must obey. The rotation matrix has four elements as given earlier and since these can take on plus or minus values, we allow for eight elements. There are first four equations of constraints necessary which 
fill the second through the fifth rows of the A matrix (AMTRI) which is a coefficient matrix. This matrix is filled in the first 8 columns with the elements of XIN as shown in the main program (Appendix $C$ ). On the righthand side is vector B (BMTRI) which is filled with the elements of matrix SS. There also are eight columns of "slack" values or variables which allow for fitting to the extent of \pm 1.0 for each of these constraints.

Because of the form of the rotation matrix (or the solution vector) all of the values of theta $(\theta)$ should be equal. Furthermore the two cosine $\theta$ values should be equivalent as should the two sine $\theta$ values. Therefore two more constraints are added to AMTRI (rows 6 and 7 ) such that the positive value of one added to the negative value of the other equal zero as evidenced by elements 6 and 7 in BMTRI. These constraints also have \pm 1.0 slack parameters, as will the successively described constraints which were later found necessary.

To return briefly to row 1 of AMTRI, the objective function which we are attempting to minimize, each element is weighted relative to its importance. Thus the larger the value of the coefficient (or A element) the more important it is that this element have a zero value. Analogously the smaller the value of element $A$, the more possible it is that this element can have a nonzero value. For this problem elements 1 through 8 were given small values (0.1), 
making them unimportant in the minimization (It will be observed that elements 2 and 8 are given values of 50.0 ; this is because it was determined that the cosine $\theta$ could not take on negative values within the limits set. That is since $\theta$ is allowed between $-\pi / 2$ and $+\pi / 2, \operatorname{cosine} \theta$ never is less than zero; these elements corresponded to negative $\operatorname{cosine} \theta$ values). Columns 9 through 16 were assigned values of 1.0 making the importance of the slack coefficient values of the first four constraints of lesser importance than the constraint coefficients themselves. Finally the last four columns of AMTRI are weighted highly so that it will be difficult for the slack values of the last two constraints to have any nonzero values, thus forcing the cosine values to be equal and the sine values to be equal.

It was found however that the solution vector elements were not equivalent when data was input, though the values were somewhat close. It was necessary therefore to add more constraints to force a better fitting. The next constraint, row 8 in AMTRI, holds the values of $\operatorname{cosine} \theta$ and sine $\theta$ from the previous iteration in columns 1 through 4 so that we have now invoked a nonlinear form into the program. Constraint 7 is forcing the solution to fit the equation $\sin ^{2} \theta+\cos ^{2} \theta=1.0$, which clearly is a nonlinear equation. The value for the slack of this coefficient is read in as WATE, the size of which defines the importance of this constraint and how fast multiple iteration will 
converge upon the desired value. If WATE is too small there will be essentially no change. However if WATE is too large, values will oscillate back and forth. The value of 5.0 seemed to work well for this problem.

Finally an eighth and last constraint was employed which would not allow the value of $\operatorname{cosine} \theta$ to exceed 1.0. The negative slack parameter was weighted very heavily so as to discourage its solution element from having anything but a zero value. Conversely the positive slack parameter was given a weighted value of zero, hopefully forcing only positive solution slack elements.

Constraints 7 and 8 were added for SIMPLX to work with only after it had solved the slightly simpler problem with 6 constraints. It would then do iterative calculations on the 9 by 24 matrix until an acceptable solution vector was obtained. Since the constraints tended to make the problem very difficult, occasionally it was necessary to weight the positive slack parameter, $\operatorname{AMTRI}(1,24)$, for constraint 8 at a value of 0.11 - a value slightly greater than element AMTRI $(1,1)$. This then returned four solution vectors of similar or equivalent value. These values were then converted into degrees and printed out.

To recapitulate how SIMPLX works then we first recognize that a matrix is formed of the coefficients for the function to be fitted (in this case to be minimized) plus the coefficients for a set of constraints, while allowing for both 
negative and positive deviations in these constraints with additional variables. An iterative technique is undertaken to fit the minimum linear form for the function. The equation which we are trying to minimize, of which matrix $A$ contains the coefficients, is the solution vector-in this case the rotation matrix. 
Electric dichroism occurs when the absorption of light by a molecule is anisotropic in an applied electric field. That is, there is a difference in absorption induced between the parallel and perpendicular polarized directions with respect to the applied electric field. This results from the fact that molecular transition moment of the molecule oriented in the electric field on the average makes a different angle with the electric vector of the incoming radiation when this radiation is polarized parallel or perpendicular to the applied orienting electric field. If the angle between the transition moment and rotating electric vector is defined as $\gamma$, the electric dichroism is then proportional to $\cos ^{2} \gamma$, the square of their scalar product.

Incorporating the extinction coefficient $\varepsilon$ we then obtain $\varepsilon=\varepsilon_{0} \cos ^{2} \gamma$, where $\varepsilon_{0}$ is the extinction coefficient value when the two above mentioned vectors are parallel. Furthermore in the absence of any field, where all values of $\gamma$ are equally probable, $\cos ^{2} \gamma$ will become the average value of $1 / 3$, as is intuitively obvious when the three dimensions are considered. It can also be shown:

$$
\overline{\cos ^{2} \gamma}=\frac{0^{\delta^{\pi} \cos ^{2} \gamma \sin \gamma d \gamma}}{0^{\rho^{\pi} \sin \gamma d \gamma}}=\frac{1}{3} \text {, so that } \varepsilon=\varepsilon_{0} / 3
$$


When an electric field is applied to a molecule which possesses electrical anistropy, a degree of bias may be introduced into the distribution function of $\gamma$. The induced and permanent dipole moment will be greater in a certain orientation if the molecular polarizability is anisotropic. This will then afford a larger interaction between the molecule and the electric field in that particular orientation. The result with regard to the distribution function $\gamma$ is that light polarized parallel and perpendicular to the field will yield different values for $\cos ^{2} \gamma$, which is dependent on the field direction. Therefore the extinction coefficients for these two orthogonal polarizations will likewise differ and it is this difference which gives the indicative dichroic effect called electric dichroism. Consequently we can define $\Delta \varepsilon=\varepsilon_{\|}-\varepsilon_{\perp}$.

Figurelo shows the various axes and angles described for the model molecule used herein which is taken to be cylindrically symmetrical with respect to its electrical, hydrodynamic, and optical properties. Hence line $\overline{\mathrm{ob}}$ is the molecular symmetry axis or the axis of the molecular dipole moment as well as the axis of excess polarizability. This axis forms angle $\beta$ with the electric field vector of the incoming radiation (line $\overline{\mathrm{Oz}}$ ) and angle $\alpha$ (which is a fixed angle) with the molecular transition moment vector (line $\overline{\mathrm{oa}}$ ).

Such a molecule will have an extinction coefficient 


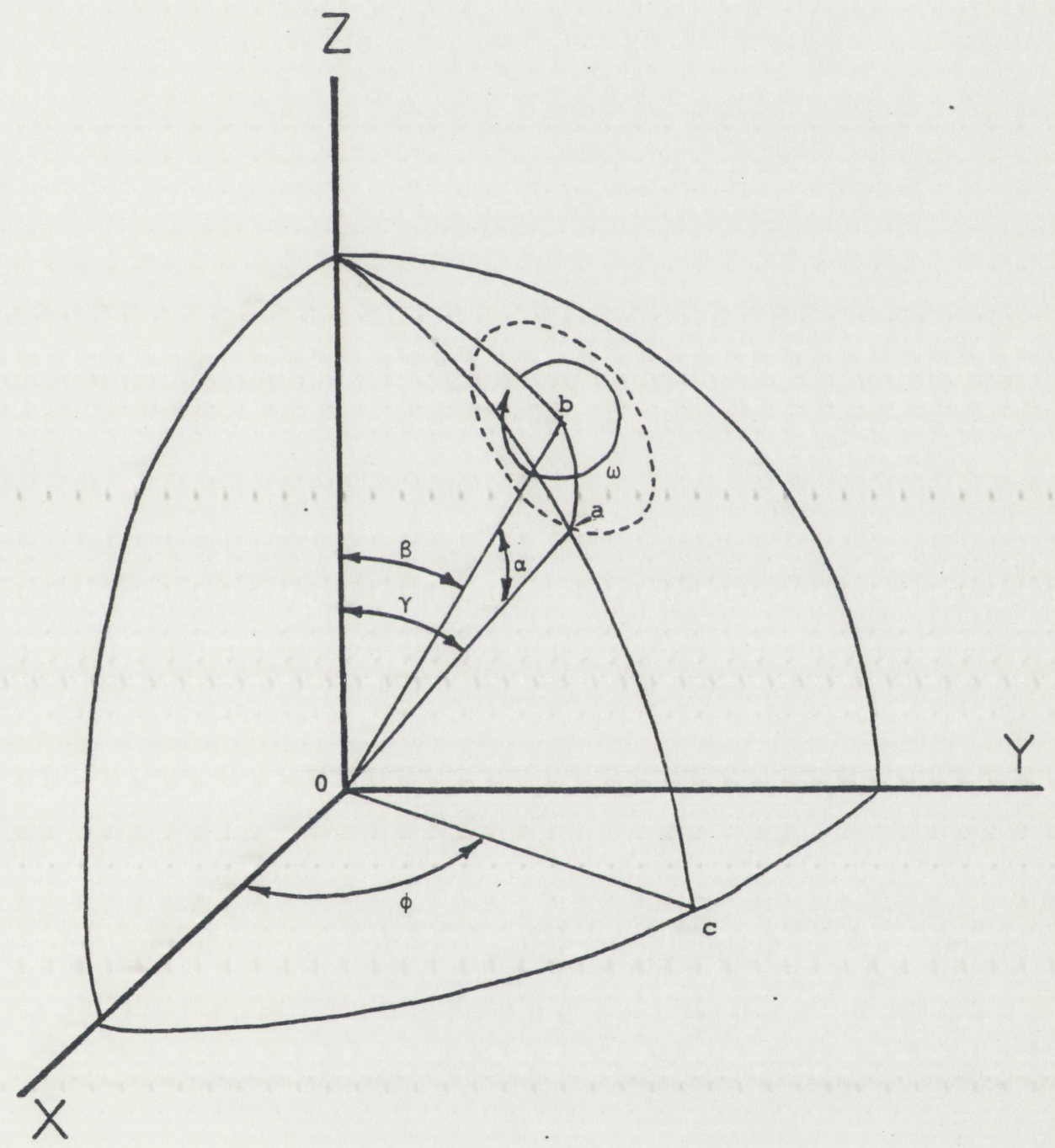

Figure 10. Model and parameter diagram 
$\varepsilon_{\|}=\varepsilon_{0} \cos ^{2} \gamma$ when light incident in the $\mathrm{x}$ direction is polarized in the $\mathrm{xz}$ plane. When the same incident light is polarized in the xy plane, the extinction coefficient is $\varepsilon_{\perp}=\varepsilon_{0}\left(\sin ^{2} \gamma \sin ^{2} \phi\right)$. In order to evaluate $\Delta \varepsilon$, the necessary trigonometric functions must be defined. Thus

$$
\cos \gamma=\cos \alpha \cos \beta+\sin \alpha \sin \beta \cos \omega
$$

so that

$$
\cos ^{2} \gamma=\cos ^{2} \alpha \cos ^{2} \beta+2 \cos \alpha \cos \beta \sin \alpha \sin \beta \cos \omega+\sin ^{2} \alpha \sin ^{2} \beta \cos ^{2} \omega
$$

Furthermore, since the molecule-field interaction energy is independent of the angles $\phi$ and $\omega$, the average value for both $\sin ^{2} \phi$ and $\cos ^{2} \omega$ (in two dimensions) equals $1 / 2$. The distribution function of these angles is indicative of the no-field case and it can also be shown:

$$
\begin{gathered}
\overline{\cos \omega}=\frac{0^{\delta^{2 \pi}} \cos \omega d \omega}{0^{2 \pi} d \omega}=0 ; \overline{\cos ^{2}}=\frac{0^{\int^{2 \pi} \cos ^{2} \omega d \omega}}{0^{2 \pi} d \omega}=\frac{1}{2} \\
\frac{\sin ^{2}}{\sin ^{2 \pi}}=\frac{0^{2 \pi} \sin ^{2} \phi d \phi}{\rho^{2 \pi} d \phi}=\frac{1}{2}
\end{gathered}
$$

From the above relations can be obtained

$$
\overline{\cos ^{2} \gamma}=\cos ^{2} \alpha \cos ^{2} \beta+1 / 2 \sin ^{2} \alpha \sin ^{2} \beta
$$

or 


$$
\begin{aligned}
\overline{\cos ^{2} \gamma} & =\cos ^{2} \alpha \cos ^{2} \beta+1 / 2 \sin ^{2} \alpha\left(1-\cos ^{2} \beta\right) \\
& =\left(\cos ^{2} \alpha-1 / 2 \sin ^{2} \alpha\right) \cos ^{2} \beta+1 / 2 \sin ^{2} \alpha \\
& =\left(\cos ^{2} \alpha-1 / 2+1 / 2 \cos ^{2} \alpha\right) \cos ^{2} \beta+1 / 2 \sin ^{2} \alpha
\end{aligned}
$$$$
\overline{\cos ^{2} \gamma}=1 / 2\left[\left(3 \cos ^{2} \alpha-1\right) \cos ^{2} \beta+\sin ^{2} \alpha\right]
$$

$$
\varepsilon_{\|}=\varepsilon_{0} \overline{\cos ^{2} \gamma} ; \quad \varepsilon_{\perp}=\frac{\varepsilon_{0}}{2} \overline{\sin ^{2} \gamma}=\frac{\varepsilon_{0}}{2}\left(1-\overline{\cos ^{2} \gamma}\right)
$$

or

$$
\begin{aligned}
\varepsilon_{\|} & =\frac{\varepsilon}{2} \circ\left[\left(3 \cos ^{2} \alpha-1\right) \cos ^{2} \beta+\sin ^{2} \alpha\right] \\
\varepsilon_{\perp} & =-\frac{\varepsilon}{2} \circ\left[1 / 2\left(3 \cos ^{2} \alpha-1\right) \cos ^{2} \beta+\frac{\sin ^{2} \alpha}{2}-1\right] \\
& =-\frac{\varepsilon}{2} \circ\left[1 / 2\left(3 \cos ^{2} \alpha-1\right) \cos ^{2} \beta-\cos ^{2} \alpha-\frac{\sin ^{2} \alpha}{2}\right]
\end{aligned}
$$

Averaging over $\beta$ and rearranging terms, an exact result for the magnitude of the dichroism is given as

$$
\frac{\Delta \varepsilon}{\varepsilon}=[9 \mathrm{R}-3]\left[\frac{3 \cos ^{2} \alpha-1}{4}\right]
$$

where $R$ equals the average value of $\cos ^{2} \beta$ and for which the mathematics are detailed elsewhere (F. Allen, dissertation, p. 30). 


\section{ELECTRIC DICHROISM INSTRUMENT AND TECHNIQUES}

The Electric Dichroism instrument at the time of its present usage has the following form as the block diagram in figure 11 shows, with a more elaborate figure given in appendix F. Monochromatic light is collimated through a rotatable linear polarizer usually set in the up and down orientation, i.e., perpendicular to the field and the photoelastic optics (which are set at an angle of 45 degrees to the applied field) on to the sample in the cell holder. The sample cuvette (10 $\mathrm{mm}$ path length) is fitted with a teflon piece to which is mounted parallel to each other two goldplated electrodes separated by $3.6 \mathrm{~mm}$. The cell holder is built such that the incident light nearly completely impinges on the sample only in the area between the electrodes so that maximum orientation of the sample can be observed. An EMI6256B photomultiplier tube is connected to a high gain amplifier and powered by a high voltage supply. This was set so that when a second polarizer is placed before the sample and rotated at 90 degrees to the first one, no light is observed. When the second polarizer is rotated back to 0 degrees, the PM tube power supply is adusted to a deflection of 60 mvolts. This then is set as maximum deflection and is considered the reference signal. (Typically the PM tube voltage was adjusted by using one's hand to cut the light.)

A pulse generator was used to determine the width of 


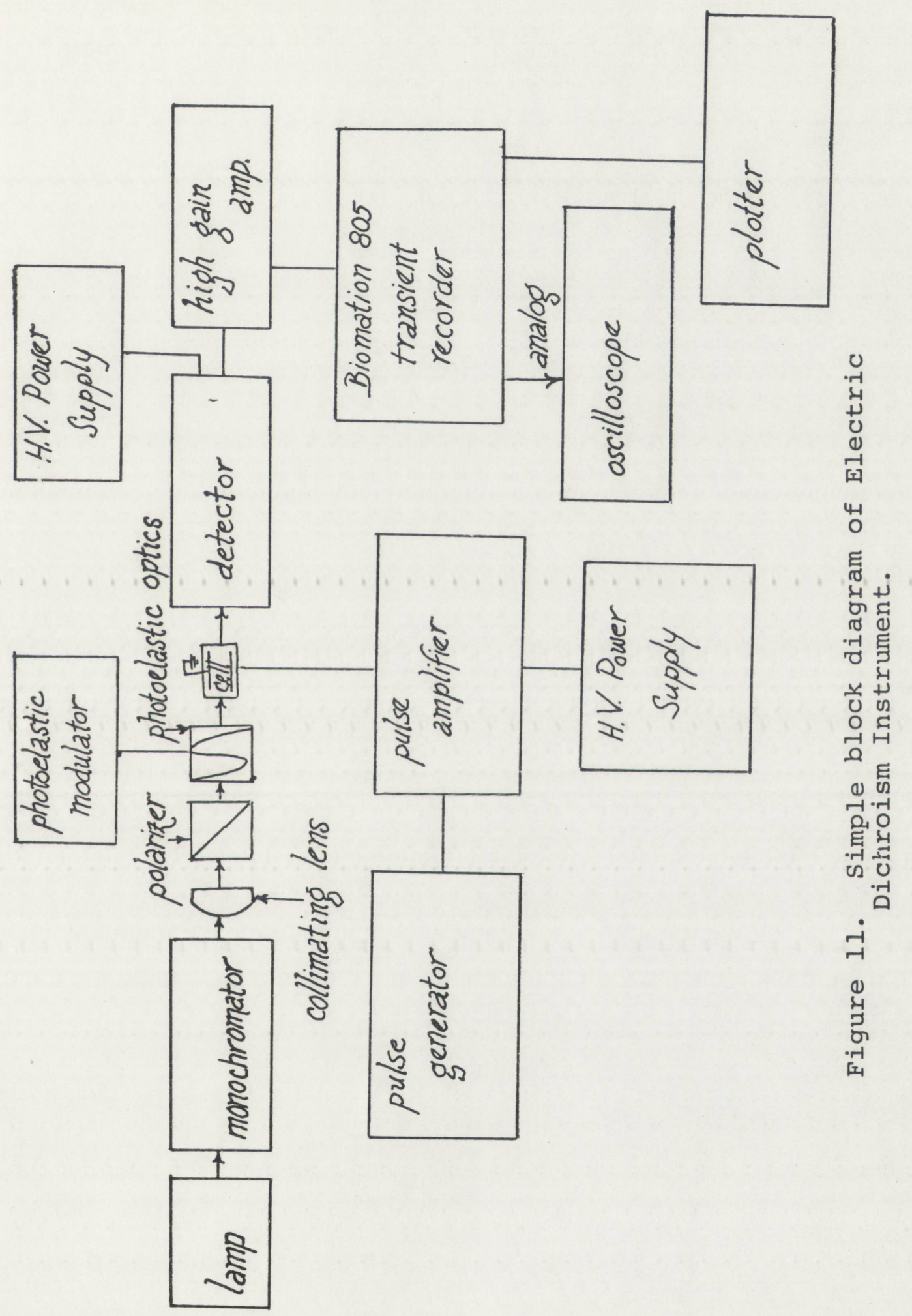


the applied voltage signal (normally 100 microseconds) and the delay time before it was applied (normally 50 microseconds). The purpose of the delay was to establish a presignal base line. The pulse generator is used to trigger a high voltage pulse across the gold-plated electrodes, thus orienting the sample in the cell.

The photoelastic modulator acts as a half wave plate. When there is no stress on it light passes directly through, however when both stressed and strained it modulates at 50 $\mathrm{kHz}$ so that it has the effect of doubling the frequency to $100 \mathrm{kHz}$. Now it modulates the plane of polarization quickly between perpendicular and parallel. This light of alternating polarization impinges on the cell and is absorbed differentially. The dichroism is thus observed as an A.C. component in the PM tube signal. Hence the greater the frequency of the modulator and the longer the duration of an applied electric pulse, the more time the light signal can be modulated (between on and off) and thus the more information the signal can carry. Essentially then, electric dichroism is recorded as a change in light intensity due to anisotropy. The electric dichroism signal is then either photographed directly off the oscilloscope or plotted out, which allows for greater accuracy. This was possible because of the capabilities provided with the Biomation 805 Waveform Recorder. Without going into undue detailed discription of the Biomation, suffice it to say that it allowed 
excellent control of signal sensitivity and also provided the possibility of two time bases concurrently. The Biomation 805 is a transient waveform recorder that digitizes a signal and stores it among 2048 points. This signal can then be output on a time base that can vary between 0.2 microseconds/ point and $100 \mathrm{milliseconds/point.} \mathrm{Thus} \mathrm{we} \mathrm{could} \mathrm{set} \mathrm{the}$ pulse and pre-pulse signal to one time base (normally 0.2 microseconds/point) and the decay (the signal after the pulse has ended) to another time base (normally 2.0 microseconds/ point). This allowed for obvious difference in signal between pulse and decay of an event.

The size of the applied pulse was easily adjusted and the observed pulse size recorded on a tektronix oscilloscope. It could also be easily arranged so that the pulse was plotted out, though as a rule this was generally not done since the accuracy provided by the oscilloscope was sufficient. Furthermore for each sample the observed voltage was maintained at a constant value since saturation could not be attained and dichroism is field strength dependent.

Interpretation of signal information was handled in one of two similar manners. Either the signal was measured from the oscilloscope (which was quicker besides being necessary while the plotting function of the Biomation was down during a 3 week period of high utility of the instrument) or it was plotted using an Houston Instruments Omnigraphic 
2000 Recorder. After calibration of both scope and plotter, the average height of the steady state dichroism was recorded, this value was divided by the full scale deflection value and multiplied by the sensitivity setting on the Biomation; this gave units of volts for the observed signal. This value was then divided by 0.25 volts $/ \mathrm{cm}$, a constant value that the high gain amplifier put out. With the observed signal now as a value of $\mathrm{cm}$. or divisions relative to or as described by the high gain amplifier, it is multiplied by the sensitivity of the same amplifier (normally set at 5 moolts $/ \mathrm{cm}$ ). Finally this value is divided by the full scale deflection of the amplifier; this was set at 60 mvolts $\left(S_{0}\right)$, while sensitivity was switched to $20 \mathrm{mvolts} / \mathrm{cm}$. This number is equivalent to $\Delta \mathrm{S} / \mathrm{S}_{0}$. Using the tables generated by the program in appendix $D$ and the solution optical density, this then is converted to $\Delta \varepsilon / \varepsilon_{0}$ and after multiplying by the extinction coefficient of the solution at that particular wavelength, we have a value for electric dichroism, $\Delta \varepsilon$.

A typical plot is shown in figure 12. It includes both the pulse and the signal trace; it is a fairly good trace as far as signal to noise ratio is concerned.

It is also somewhat noteworthy to indicate certain precautions which are wise to follow in using the electric dichroism instrument. Once it is completely turned on and operable the following sequence is advised when pulsing a 


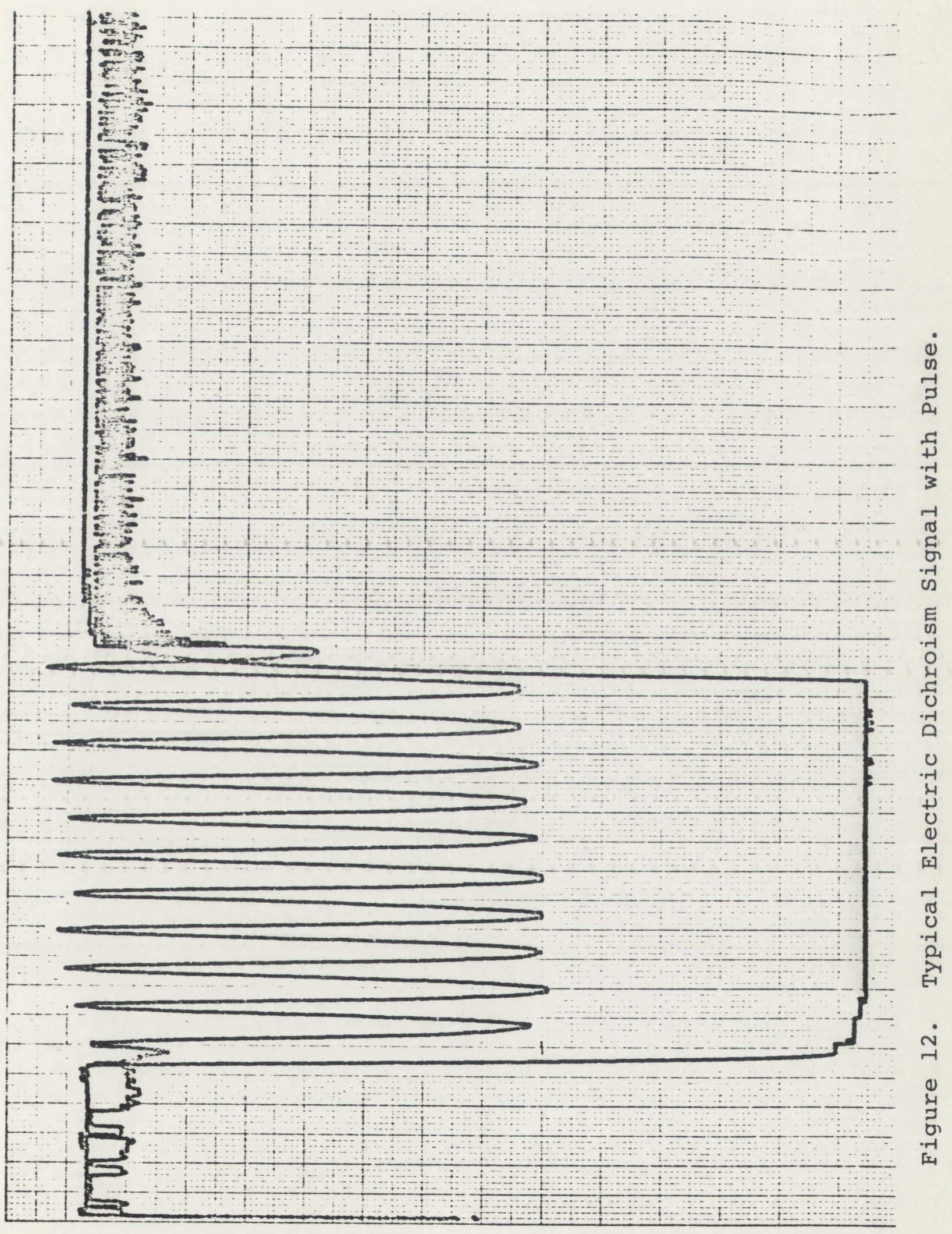


sample and recording the signal: before the sample to be studied is pulsed, once the desired high voltage is turned on, the circuit to the cell should be closed and the system pulsed as such a few times. Alternatively a load resistor can be placed across the electrode contacts and the system pulsed a few times with the circuit open to the cell. This is done to prevent multiple or even single triggering when the switch to the cell is initially thrown open without ever pulsing the sample. This helps to discourage sample degradation and possible cell explosion or sparking. It is also necessary to insure that the cell with the electrodes inserted is dry of any excess solution and clean of fingerprints-this can also cause sparking.

Once the system has been thus "cleared" and a sample is ready to be pulsed, the high voltage switch is thrown open to the cell, quickly pulsed, and the high voltage switch immediately thrown off, or closed to the cell. This is advised in case any of the tubes (especially the 5D21) locks up-another good way to blow a cell. Now with the high voltage closed to the cell the signal on the scope can be plotted out without upsetting the Biomation signal.

(The Electric Dichroism instrument is described in elaborate detail in the dissertation of William Rahe, The University of New Mexico, 1977) 


\section{EXPERIMENTAL SECTION}

\section{Results with tRNA}

Due to the low sensitivity of our electric dichroism instrument, it was impossible to obtain any solution data for tRNA. This is primarily because the signal to noise ratio was extremely low and the supposed dichroism must be somewhat small. By improving the optical section of the instrument this problem could be rectified; that is, by using a light source of considerably higher intensity, with a better collimating lens, and more sensitive photomultiplier tube, a smaller PM tube voltage would be required for observing the phenomenon being studied. This would improve the signal to noise ratio so that the information carried in the signal would be more accessible. After this improvement is made, tRNA could then hopefully be studied.

\section{Results with DNA}

The electric dichroism of T2 DNA was easily observed, giving a clean signal (with a high signal to noise ratio) in the wavelength region studied (220-290 nm). A sample of T2 purchased from Worthington Biochemicals was dissolved to a concentration of about $0.024 \mathrm{mg} / \mathrm{ml}$ in a $\mathrm{pH} 8$ buffer (a $\mathrm{pH}$ at which DNA maintains its native conformation without needing higher salt concentrations (54)) of $0.1 \mathrm{mM} \mathrm{Na}{ }_{2} \mathrm{HPO}_{4}$ and $0.01 \mathrm{mM} \mathrm{NaH} 2 \mathrm{PO}_{4}$ and then sonicated with a VWR Biosonik 
IV at maximum power for 20 seconds/ml of solution while in an ice bath (to prevent denaturation from heating) so as to obtain more uniform particle size. Electric dichroism signals were taken at a constant voltage of approximately $14.0 \mathrm{kv} / \mathrm{cm}$. A 100 microsecond pulse of $6.5 \mathrm{kv}$ was normally applied with $5.0 \mathrm{kv}$ observed across the sample. It was noted that the sample was pulsed at least 10-15 times without degradating it.

A plot of $\Delta \varepsilon$ versus wavelength for T2 DNA is shown in ficure 13. Using the computer program in appendix $C$, the extinction coefficients obtained from this graph and that of the ultraviolet absorption spectra of the same solution were used to calculate the extinction coefficients for the perpendicular and parallel direction. These values were normalized and then compared to the normalized optical densities obtained from the ultraviolet absorption spectra of an oriented T2 DNA film at various humidities. The results are tabulated in table 1; the significance of the results is explained in the computer treatment section. Essentially it is indicated that the structure, as described by the extinction coefficients, is the same for $\mathrm{T} 2$ DNA in oriented film as it is in solution and that a rotation through an angle of zero degrees (no rotation necessary that is) forces the solution and film data to be equivalent. This remains the case for each of the relative humidities under which film spectra were compared, 


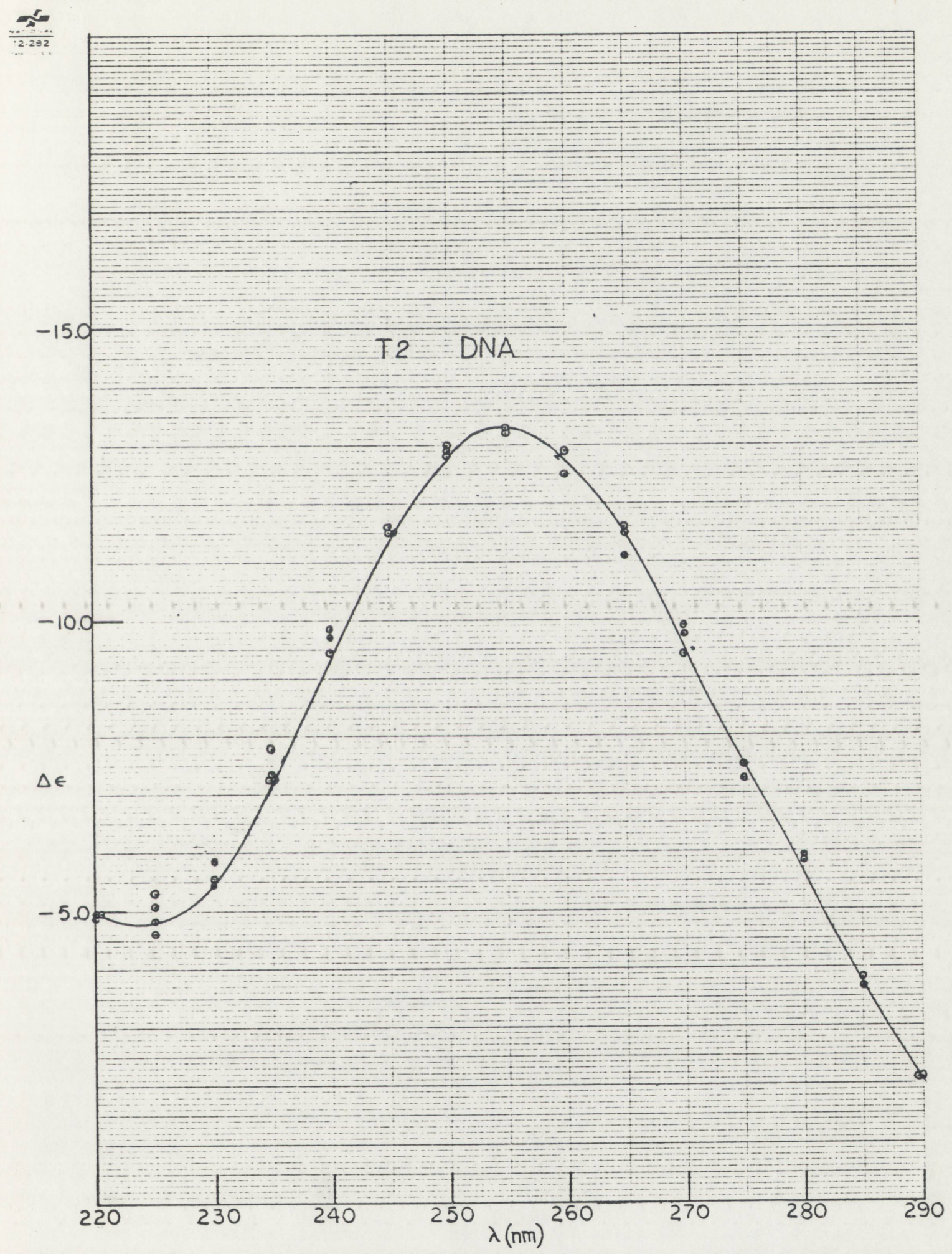

Figure 13. Steady State Electric Dichroism of T2 DNA. Concentration of $0.0238 \mathrm{mg} / \mathrm{ml}$ in $0.1 \mathrm{mM}$ sodium phosphate buffer, $\mathrm{pH}$ 8. Constant voltage of $14.0 \mathrm{kv} / \mathrm{cm}$. 
T2

$\begin{array}{llllll}92 \% \uparrow & 0.70388 & 0.49530 & 0.49530 & 0.69942 & 1 \\ 92 \%+ & 0.12033 & 0.12534 & 0.12534 & 0.12033 & 1 \\ 75 \% \uparrow & 0.09487 & 0.07113 & 0.07113 & 0.09487 & 1 \\ 75 \%+ & 0.17583 & 0.12425 & 0.12425 & 0.17132 & 1 \\ 66 \% \uparrow & 0.0 & 0.0 & 0.0 & 0.0 & 1 \\ 66 \%+ & 0.33804 & 0.23952 & 0.23952 & 0.33804 & 1 \\ 56 \% \uparrow & 0.01978 & 0.0 & 0.0 & 0.01978 & 1 \\ 56 \%+ & 0.01978 & 0.0 & 0.0 & 0.0 & 1 \\ 34 \% \uparrow & 0.01978 & 0.0 & 0.0 & 0.01978 & 1 \\ 34 \% \downarrow & 0.01978 & 0.0 & 0.0 & 0.01978 & 1 \\ 0 \% 4 & 0.01978 & 0.0 & 0.0 & 0.01978 & 4 \\ 0 \% \downarrow & 0.01978 & 0.0 & 0.0 & 0.01978 & 1\end{array}$

Calf Thymus

$\begin{array}{rrrrrr}90 \% & 0.05595 & 0.01261 & 0.01261 & 0.02798 & 1 \\ 60 \% & 0.07402 & 0.01250 & -0.01250 & 0.05234 & 4\end{array}$

poly (
$60 \%$
0.0
0.0
0.0
0.0
1

polycytidylic acid

$50 \% \quad 0.0$

0.0

0.0

0.0

1

Table 1. Results from computer treatment of data for indicated nucleic acids. (The per centage number corresponds to the relative humidity of the film; for $\mathrm{T} 2$ the arrow following this indicates either increasing or decreasing humidity.) 
especially to within the degree of accuracy ( 2 decimal places) at which the extinction coefficients were obtained.

A tabulation of the raw imput data is given in Appendix E, including the extinction coefficients of the ultraviolet solution spectra and electric dichroism, plus optical densities of the oriented film data for the perpendicular and parallel directions at the various relative humidities. Similar data for the other nucleic acids is also included in the appendix.

The electric dichroism of Calf Thymus DNA was similarly observed with generally a high signal/noise ratio in the wavelength region studied $(240-285 \mathrm{~nm})$. Calf Thymus DNA was purchased from Worthington Biochemicals. It was dissolved to a concentration of $0.050 \mathrm{mg} / \mathrm{ml}$ in $0.1 \mathrm{mM} \mathrm{Tris}$ $(\mathrm{pH} 8)$ - a solution of the same solvent as the T2 gave similar electric dichroism results - and likewise sonicated for 20 seconds/ml. Electric dichroism signals were taken at a constant voltage of approximately $6.7 \mathrm{kv} / \mathrm{cm}$. A 100 microsecond pulse of $3.0 \mathrm{kv}$ was applied with $2.4 \mathrm{kv}$ observed across the sample.

A plot of $\Delta \varepsilon$ versus wavelength for Calf Thymus DNA is shown in figure 14. Comparison was done for two humidities of oriented film of DNA and solution. Both gave an angle of zero rotation required to force equivalence of the solution and film data, just as did T2 DNA. 


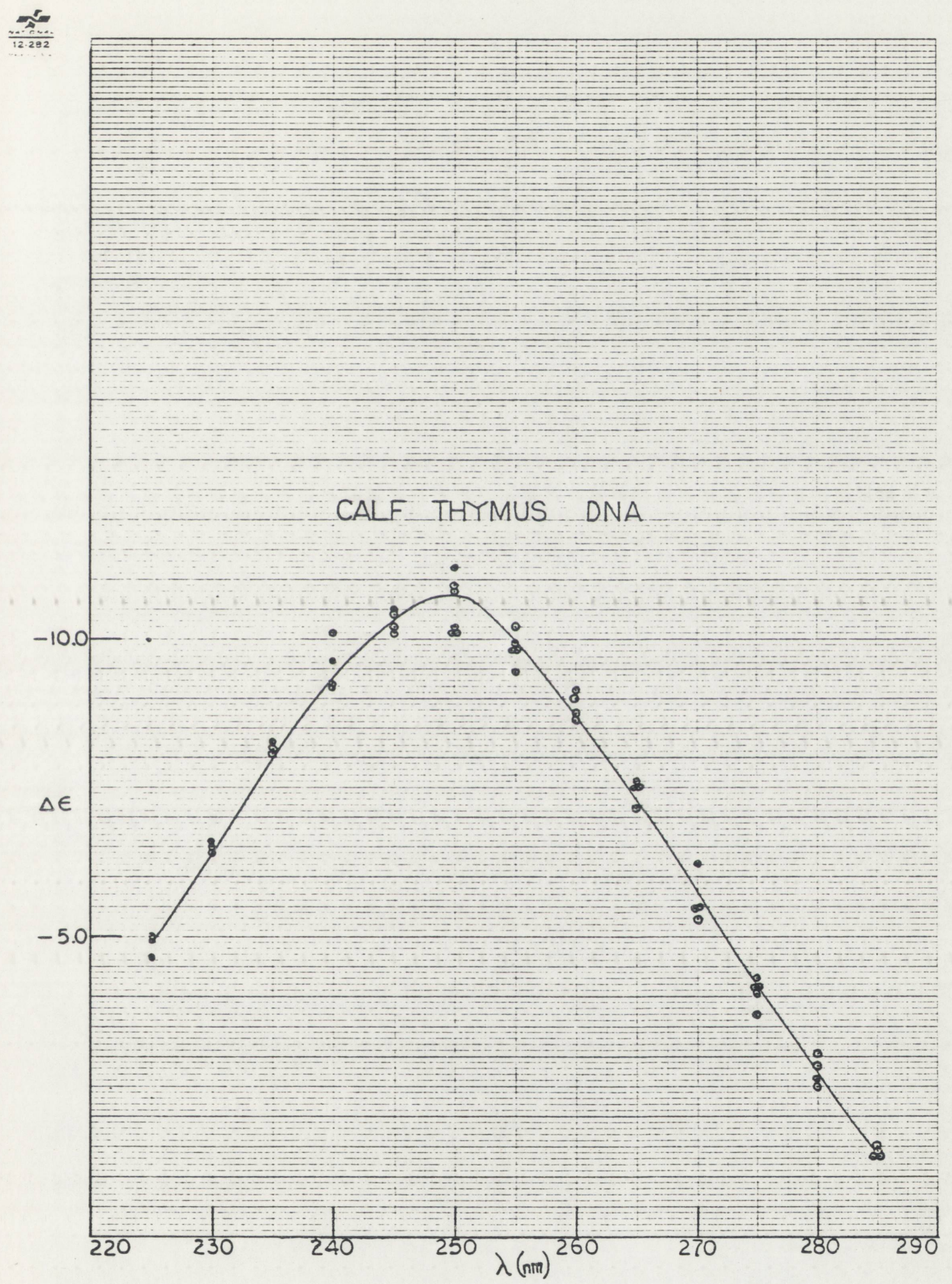

Figure 14. Steady State Electric Dichroism of Calf Thymus DNA. Concentration of $0.050 \mathrm{mg} / \mathrm{ml}$ in $0.1 \mathrm{mM}$ Tris, $\mathrm{pH} 8$. Constant voltage of $6.7 \mathrm{kv} / \mathrm{cm}$. 


\section{Results with Polynucleotides}

Poly(dAT:dAT) gave the stronger electric dichroism signal of the polynucleotides studied. An excellent signal to noise ratio was observed through most of its studied absorption wavelength region (230-290 nm). Poly (dAT) copolymer was purchased from Calbiochem and dissolved in doubly distilled water to a concentration of $0.023 \mathrm{mg} / \mathrm{ml}$ and then sonicated. Electric dichroism signals were taken at a constant voltage of $14.0 \mathrm{kv} / \mathrm{cm}$. Again a $100 \mathrm{micro-}$ second pulse of $6.4 \mathrm{kv}$ was applied to the sample, while $5.0 \mathrm{kv}$ was observed.

A plot of $\Delta \varepsilon$ versus wavelength for poly ( $\mathrm{AAT}: \mathrm{dAT}$ ) appears in figure 15. Only one relative humidity was available for an oriented film of the sample. The computer results gave excellent agreement between the four output parameters. Again the required angle of rotation was zero degrees for fitting the film data to the solution data.

Finally polycytidylic acid (poly C) was studied and found to exhibit electric dichroism, though the observed signal was generally poor especially by comparison to poly (dAT:DAT) and the data had more scatter than that of any of the other nucleic acids studied. A sample of poly C (obtained from Calbiochem) was dissolved in doubly distilled water to a concentration of $0.050 \mathrm{mg} / \mathrm{ml}$ and then sonicated. Electric dichroism signals were taken at a 


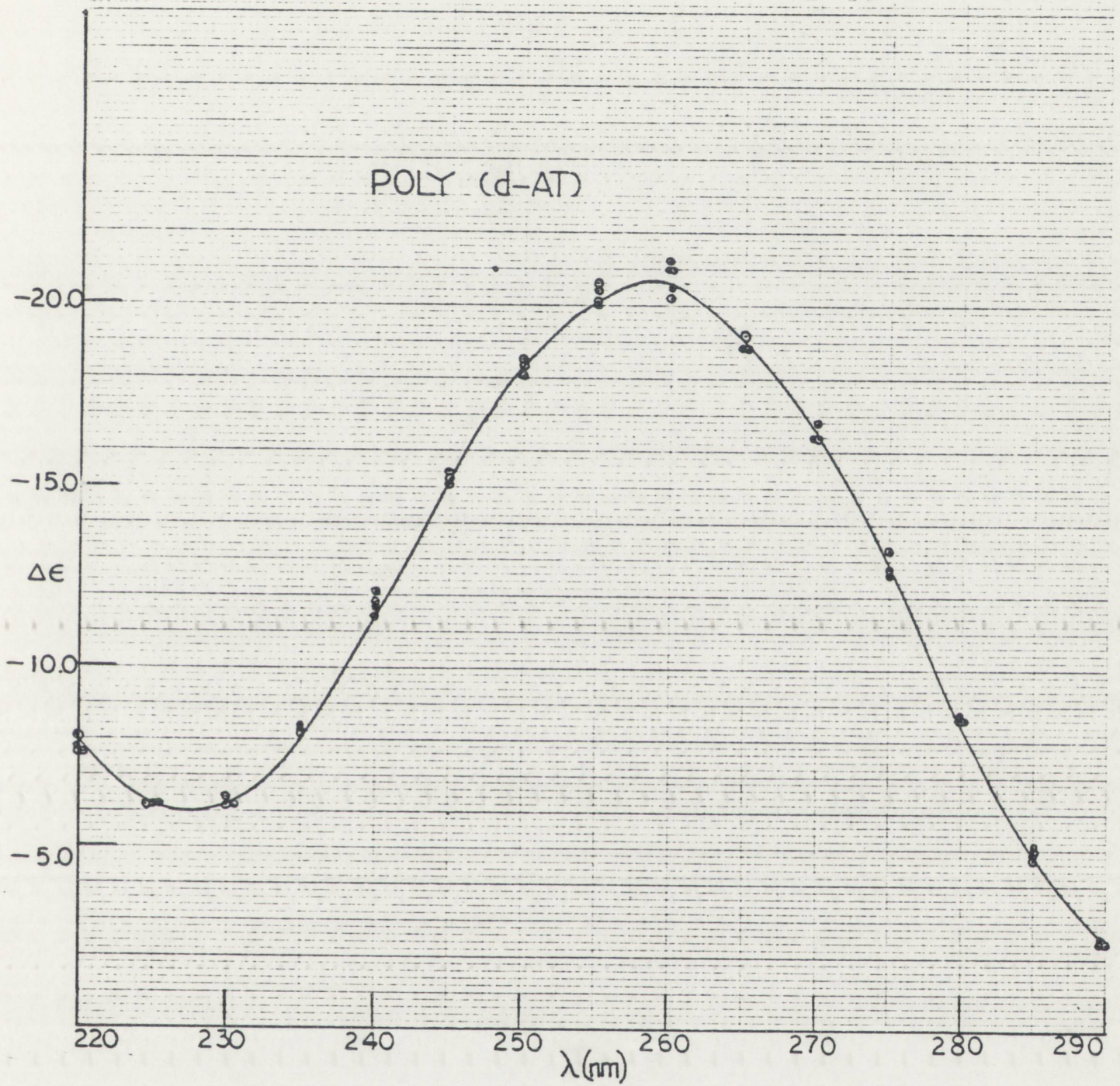

Figure 15. Steady State Electric Dichroism of poly (dAT) copolymer. Concentration of $0.0228 \mathrm{mg} / \mathrm{ml}$ in distilled water, $\mathrm{pH}$ 7. Constant voltage of $14.0 \mathrm{kv} / \mathrm{cm}$. 
constant voltage of $14.8 \mathrm{kv} / \mathrm{cm}$. And again a 100 microsecond pulse of $7.0 \mathrm{kv}$ was applied while $5.3 \mathrm{kv}$ was observed. Because of the size of the observed voltage pulsed through the sample and the small value of dichroism observed, it is inferred that poly $C$ does not have a very rigid helical form. Indeed a similar applied voltage for poly ( $\mathrm{dAT}-\mathrm{dAT})$ and DNA obtains a much greater value for the dichroism indicating that these samples are more easily oriented in an electric field. A plot of $\Delta \varepsilon$ versus wavelength for poly $\mathrm{C}$ is shown in figure 16. Somewhat surprisingly the computer results for poly $\mathrm{C}$ also gave excellent agreement between the output parameters. The required angle of rotation was zero degrees for forcing the film data into the solution data.

Interestingly enough, for both poly $\mathrm{C}$ and Calf Thymus DNA, the absorption maxima from the electric dichroism spectra and that from the ultraviolet absorption spectra are not coincident, but the data are yet shown to be equivalent, attesting to essentially the same tertiary structure.

\section{Comparison calculations with flow dichroism}

Results for linear dichroism using flow orientation were digitized and found to be equivalent to those for electric dichroism. Thus the predicted angle of rotation as inferred from preceeding results is calculated to be zero degrees with precise agreement between the four fit- 


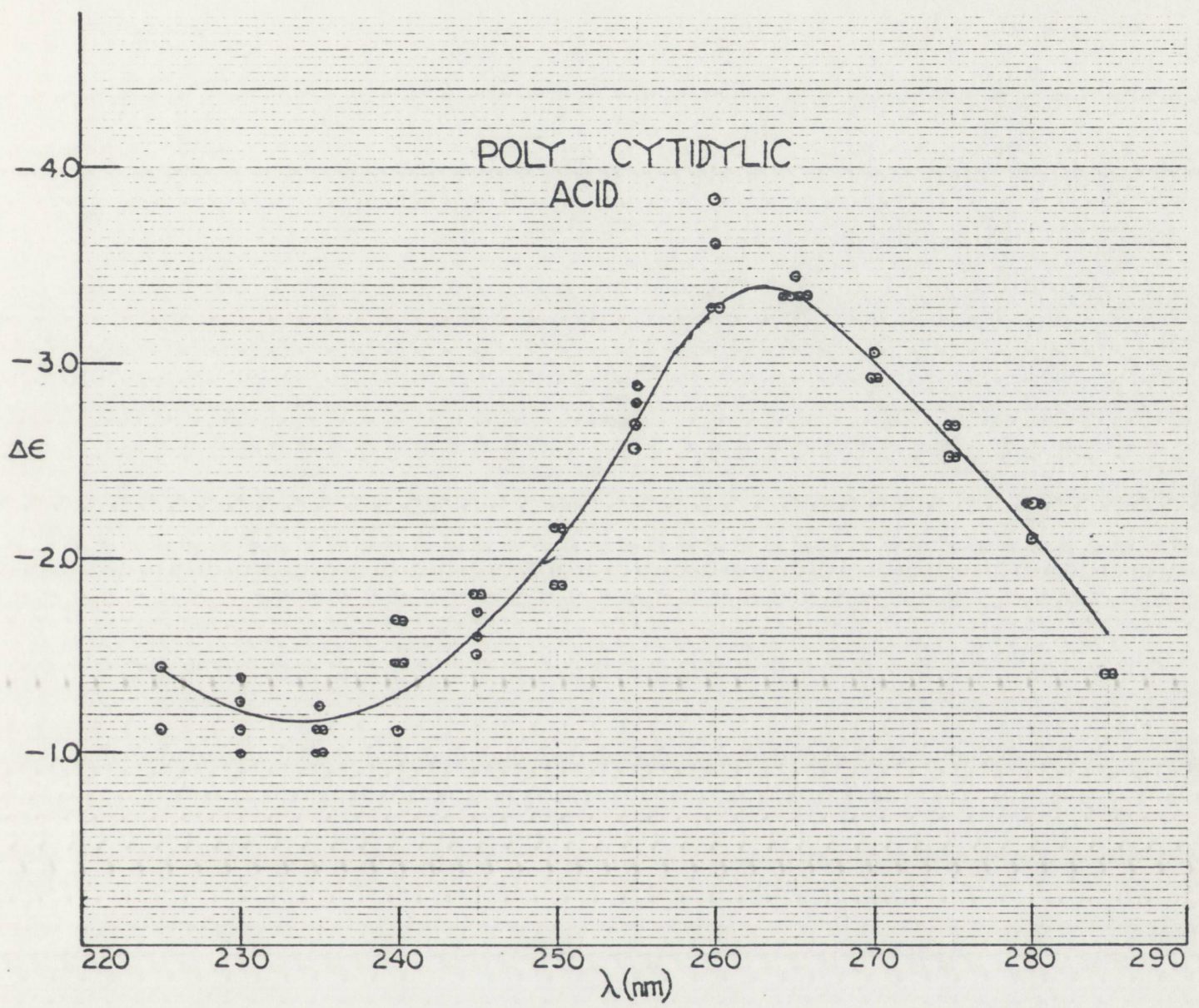

Figure 16. Steady State Electric Dichroism of polycytidylic acid. Concentration of $0.030 \mathrm{mg} / \mathrm{ml}$ in distilled water, $\mathrm{pH} 7$. Constant voltage $14.8 \mathrm{kv} / \mathrm{cm}$. 
ting parameters.

5. Results for Relaxation of Electric Dichroism

Relaxation of dichroism is generally described by the equation

$$
S(t)=S^{\prime} e^{-t / \tau}
$$

where $S(t)$ is the relaxation signal, $S^{\prime}$ the steady state signal, $t$ is the time, and $\tau$ is the relaxation time constant. By plotting $\ln \left(S(t) / S^{\prime}\right)$ versus $t$ one should get a straight line with a slope equal to $-1 / \tau$, at least this is expected for a relaxing system of one component. The data analyzed here were treated as a relaxing system of two components having the general form:

$$
S(t)=\alpha_{1} e^{-t / \tau_{1}}+\alpha_{2} e^{-t / \tau_{2}}
$$

The data were plotted and analyzed using the peeling method. In this manner the curves were evaluated to give two relaxation time from which are obtained the rotational diffusion constants $\theta_{1}$ and $\theta_{2}$, since $\tau=1 / 6 \theta$. Plots from the decay curves for the nucleic acids are given in figures 17-19 and are composites taken from the data of several electric dichroism signals. By changing the second time base of the Biomation, accurate data was accessible throughout the entire region of decay. However it is noted that due to a low signal/noise ratio it was impossible to obtain reliable relaxation data for polycyticylic acid. 


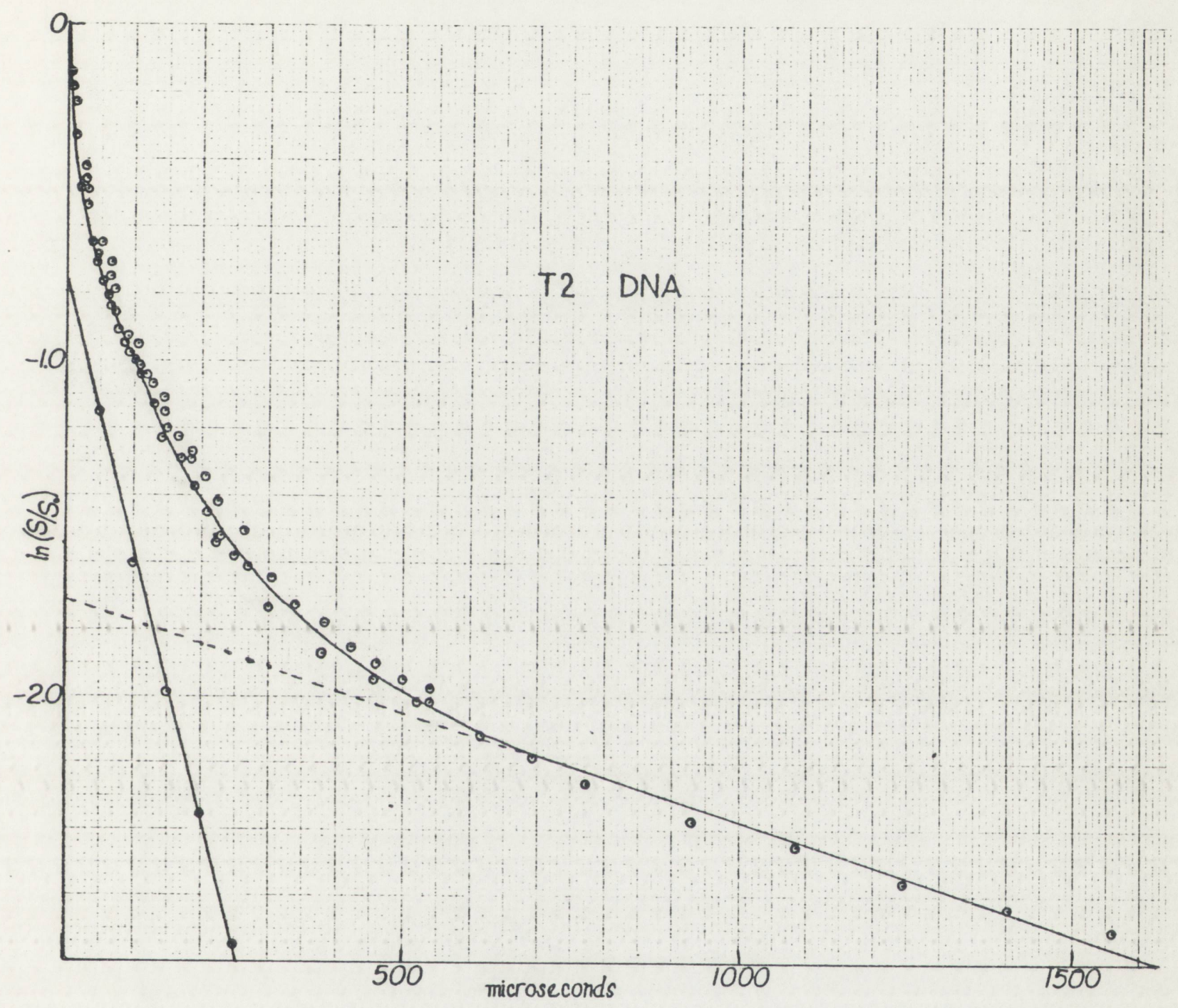

Figure 17. Relaxation plot of T2 DNA. Composite from 5 spectra at constant field strength of $6.2 \mathrm{kv} / \mathrm{cm}$. Rotary diffusion constants $\theta_{1}=1340 / \mathrm{sec}, \theta_{2}=112 / \mathrm{sec}$ and relaxation times $\tau_{1}=124 \mu \mathrm{sec}, \tau_{2}=1.48 \mathrm{msec}$. 


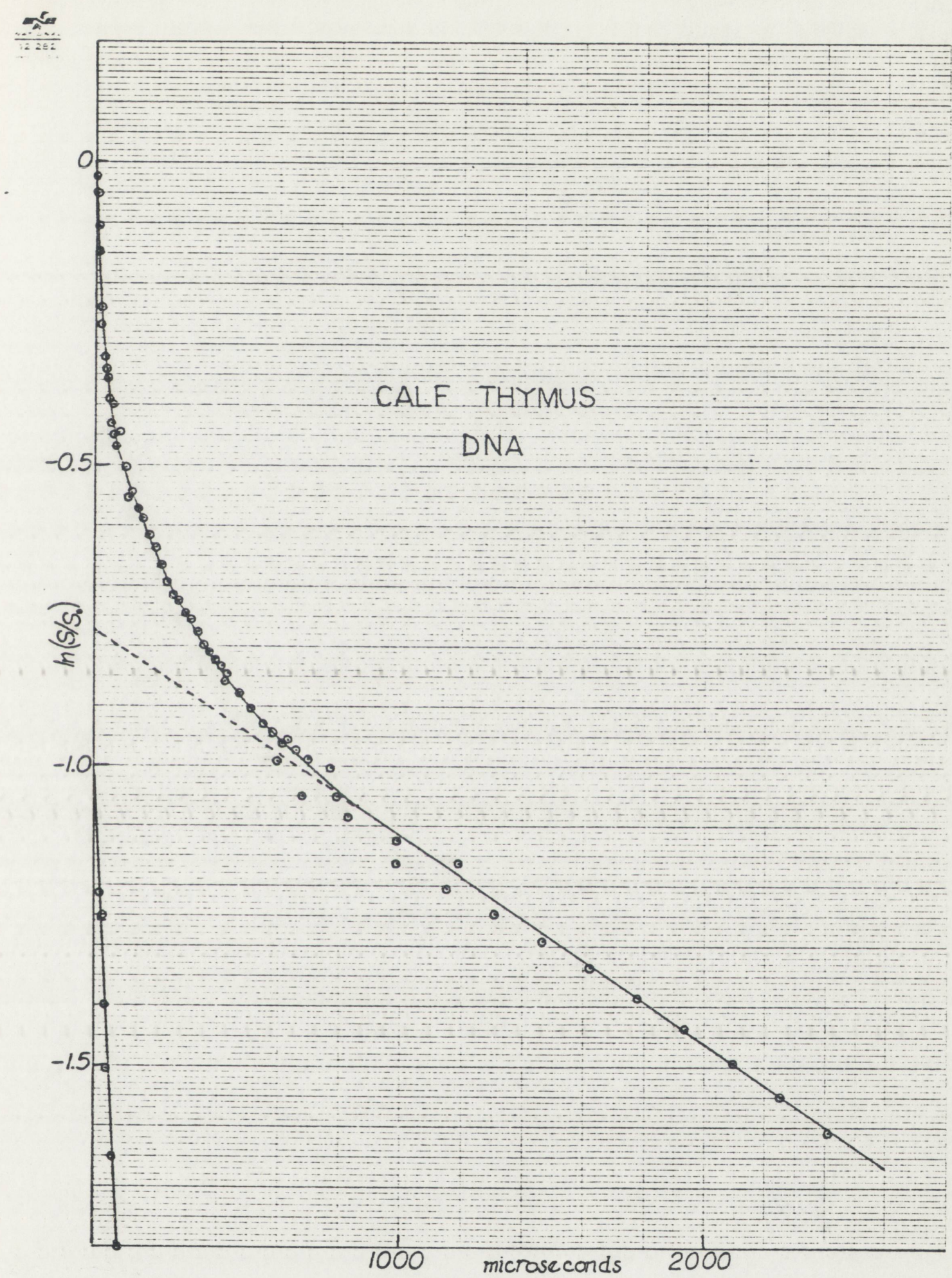

Figure 18. Relaxation plot of Calf Thymus DNA. Composite from 6 spectra at constant field strength of $5.1 \mathrm{kv} / \mathrm{cm}$. Rotary diffusion constants $\theta_{1}=1670 / \mathrm{sec}, \theta_{2}=57.5 / \mathrm{sec}$ and relaxation times $\tau_{1}=100 \mu \mathrm{sec}, \tau_{2}=2.9 \mathrm{msec}$. 


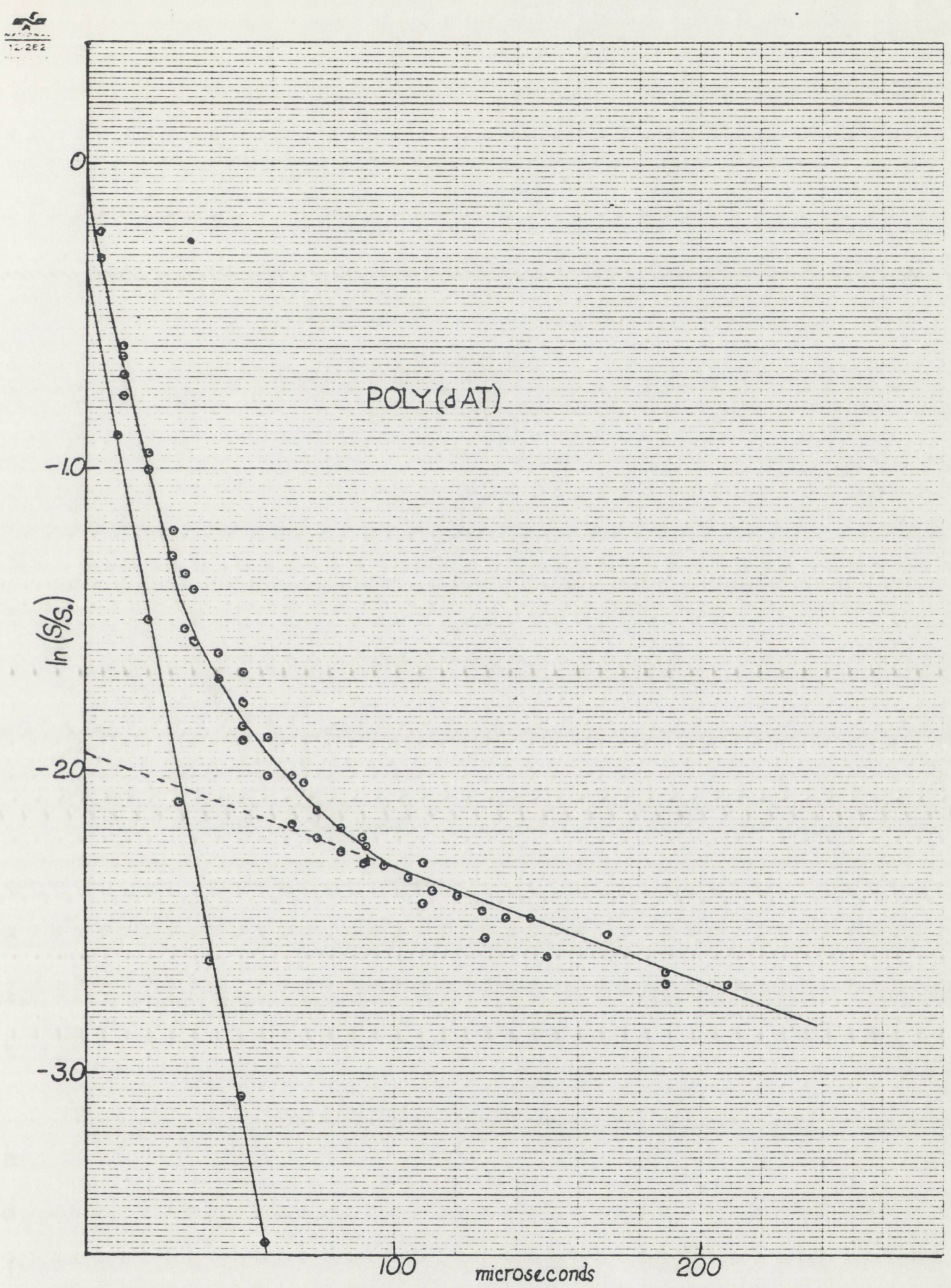

Figure 19. Relaxation plot of poly (dAT-dAT). Composite from 4 spectra at constant field strength of $5.6 \mathrm{kv} / \mathrm{cm}$. Rotary diffusion constants $\theta_{1}=9279 / \mathrm{sec}, \theta_{2}=625 / \mathrm{sec}$ and relaxation times $\tau_{1}=18 \mu \mathrm{sec}, \theta_{2}=267 \mu \mathrm{sec}$. 


\section{DISCUSSION OF RESULTS}

The probable hyperchromism observed during drying

of T2 DNA apparently has no affect on the axial nature of the molecule as implied by the calculated results. The greater increase in absorption for the parallel polarization over the perpendicular polarization resulting from tilting of the base planes, which is a reversible phenomenon (50) and hence the increased dichroic ratio curiously enough is shown not to affect the basic overall axial structure. Thus the apparent tilting of bases upon drying, or the reverse process of reordering upon hydration does not alter the direction of molecular dipole moment. The zero degrees calculated as the angle necessary to rotate the film data into the solution data indicates the dipole moment is along the helical axis in the DNA. Any base tilting allowed at different humidities must be such that the direction of the dipole moment does not change-or indeed if it does change it must be very slight. This also indicates there is no transverse component to the dipole moment.

Additionally since the film stretch axis equals the axis of the molecule when oriented electrically and hydrodynamically it becomes valid to say that the physically (mechanically), hydrodynamically, and electrically oriented axes are symmetrical and coincident. Thus the model used as a premise in the theory of linear dichroism is born out and verified. These results corroborate the 
results D. Gray arrived at concerning T2 DNA. That is, because the hydration and dehydration of DNA were found to be reversible (52), it implied that axial DNA alignment was not altered. It can reasonably well be assumed that similar results would be observed for T5 DNA of which oriented films were also studied over the same range of humidities.

Though only two different relative humidities were reported for oriented film spectra of Calf Thymus DNA, the results substantiate the previous remarks. It then seems reasonable to speculate that had tRNA crystal and electric dichroism data been attainable, the same results would have been calculated, that is that tRNA maintains the same tertiary structure in the crystal as it does in solution. This is hopefully something that can be specifically looked at in the future.

The decay curves have been shown to yield two relaxation components, one that relaxes very quickly and a second which is a bit more indicative of the overall structure that relaxes considerably slower. It is this longer relaxation component that constitutes the greater part of the decay curve. The values of $\theta_{1}$ and $\theta_{2}$ for the Calf Thymus are shown to be in good agreement with literature values as shown in Table 2 . 
Table 2. Rotation Diffusion Coefficients for Calf Thymus DNA

\begin{tabular}{|c|c|c|c|c|}
\hline Medium & $\begin{array}{l}\text { field strength } \\
(\mathrm{kv} / \mathrm{cm})\end{array}$ & $\left(\sec ^{\theta_{1}}-1\right)$ & $\stackrel{\theta_{2}}{\left(\sec ^{-1}\right)}$ & Source \\
\hline $\begin{array}{l}\text { tris buffer } \\
\text { pH } 8\end{array}$ & 6 & - & 33.2 & Shirai \\
\hline $\begin{array}{l}1 \mathrm{mM} \mathrm{NaCl} \\
\mathrm{pH} 6.5\end{array}$ & 6 & 2000 & 88 & Houssier \\
\hline water & 5 & 555 & 18 & Golub \\
\hline $\begin{array}{l}\text { tris buffer } \\
\text { pH } 8\end{array}$ & 5.1 & 1670 & 57.5 & this wo \\
\hline
\end{tabular}

The values for T2 DNA, $\theta_{1}=1340 \mathrm{sec}^{-1}$ and $\theta_{2}=112 \mathrm{sec}^{-1}$ are also of the same order of magnitude as Calf Thymus DNA. Poly ( $\mathrm{dAT}-\mathrm{dAT})$ however exhibits much faster relaxation with rotary diffusion constants of $\theta_{1}=9270 \mathrm{sec}^{-1}$ and $\theta_{2}=$ $625 \mathrm{sec}^{-1}$.

The graph in figure 20 shows that the limiting value for $1 / E$ is too low for poly (dAT-dAT). The plot for Calf Thymus DNA however gives approximately the same limiting value for $1 / E$ as the literature, indicating Calf Thymus DNA is in the native state.

Finally it has been shown that the instrument built in our lab operates correctly in that it can reproduce literature values. The work of this thesis then, provides a strong foundation that substantiates further, and perhaps more intensive, research along these same lines.

In summation, the primary results presented in this thesis consist in formulating a methodology for rotating 


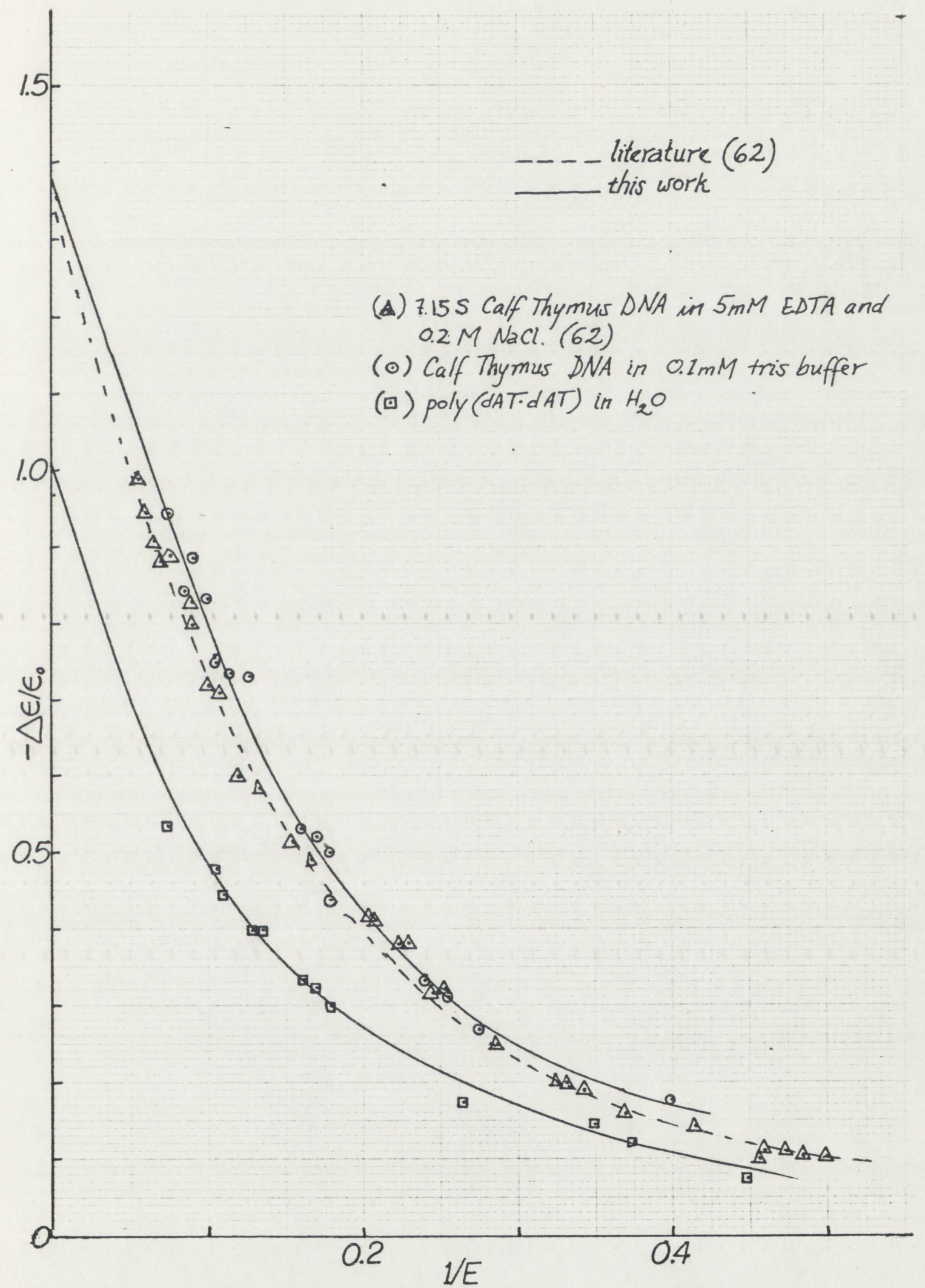

Figure 20. Steady State dichroism data graphed versus $1 / \mathrm{E}$. 
crystal spectra into solution spectra. This matrix methodology is described in detail in section II. A simplified form of this process was invoked to a similar end for rotating oriented film spectra into solution spectra. Having shown with experimental data that the simpler rotation methodology indeed works and that the more intricate analogous operation works with simulated data, we can therefore say with assurance that the Eulerian rotation matrix methodology will be of significant value with appropriate experimental data for discussing similarities between crystal and solution tertiary structure.

Secondly the computer results indicating that DNA aligns along its helical axis is a significant contribution, especially in that this axis is shown to be coincident with the mechanical, electrical, and hydrodynamical axes. of final consequence is the matter of having shown that our electrical dichroism instrument can yield valid numerical parameters. This is indicated by the instrument's ability to duplicate values that have been published previously in the literature. 
Appendix A

Main Program with LINIT

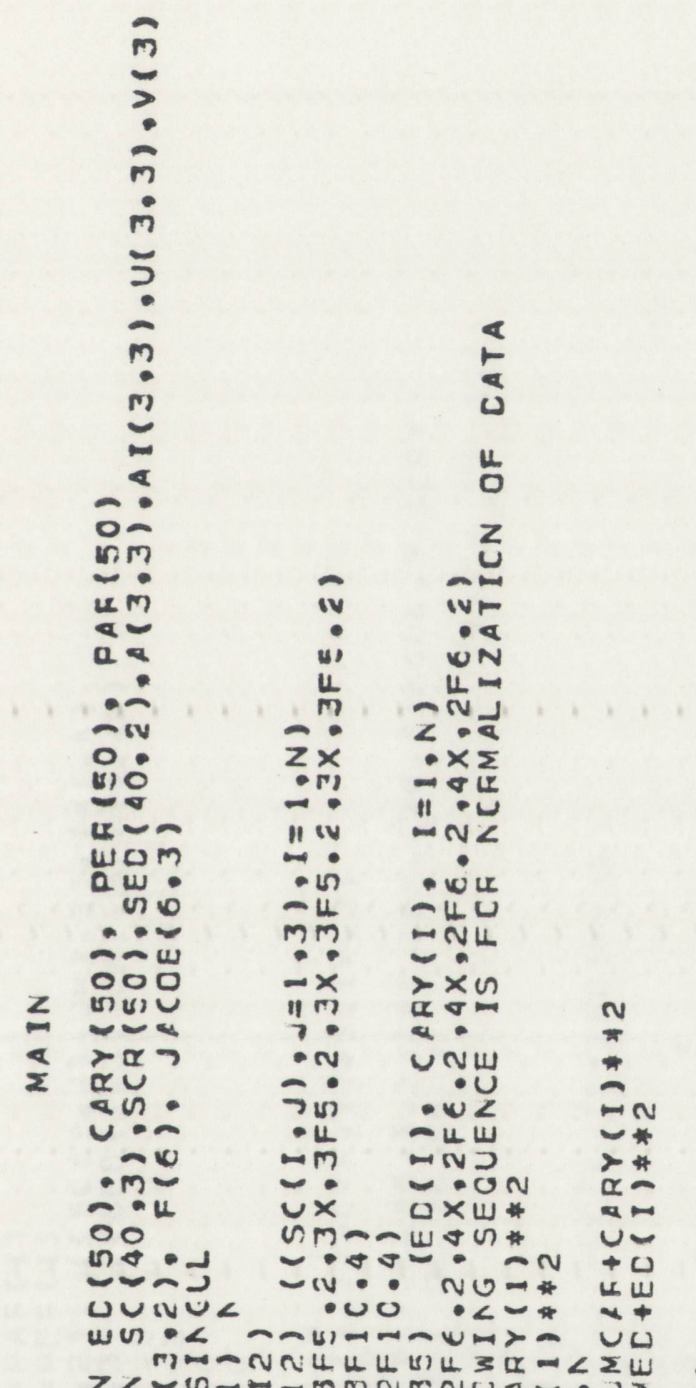

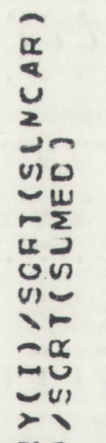

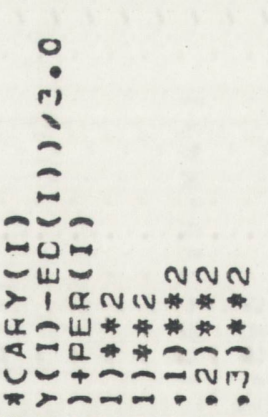

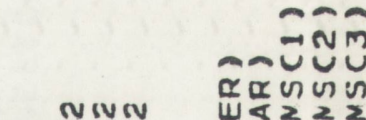

เข

แष 222

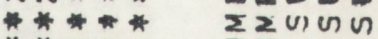

* * ว

- $-\pi m$

느:-

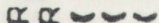

แ⿺乚U

a

$++++$

(I)

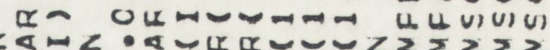

<बル

- MNNmNu\&-

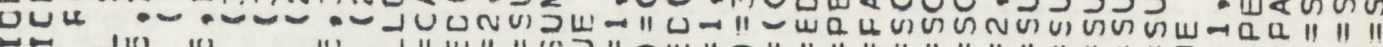
(U) Uأ |

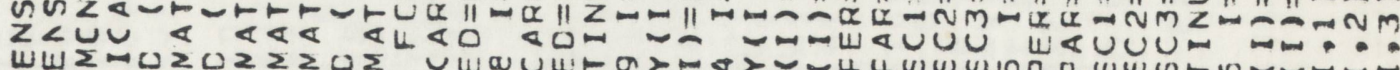
Wய2

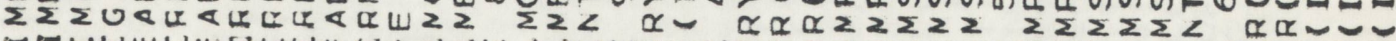

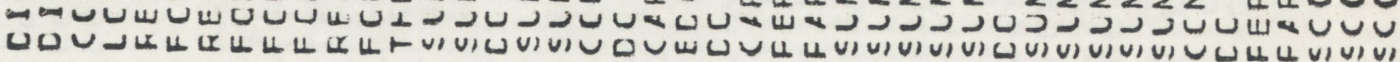

$\rightarrow$ ovm un

$\infty$ a

$\checkmark$
(5) 


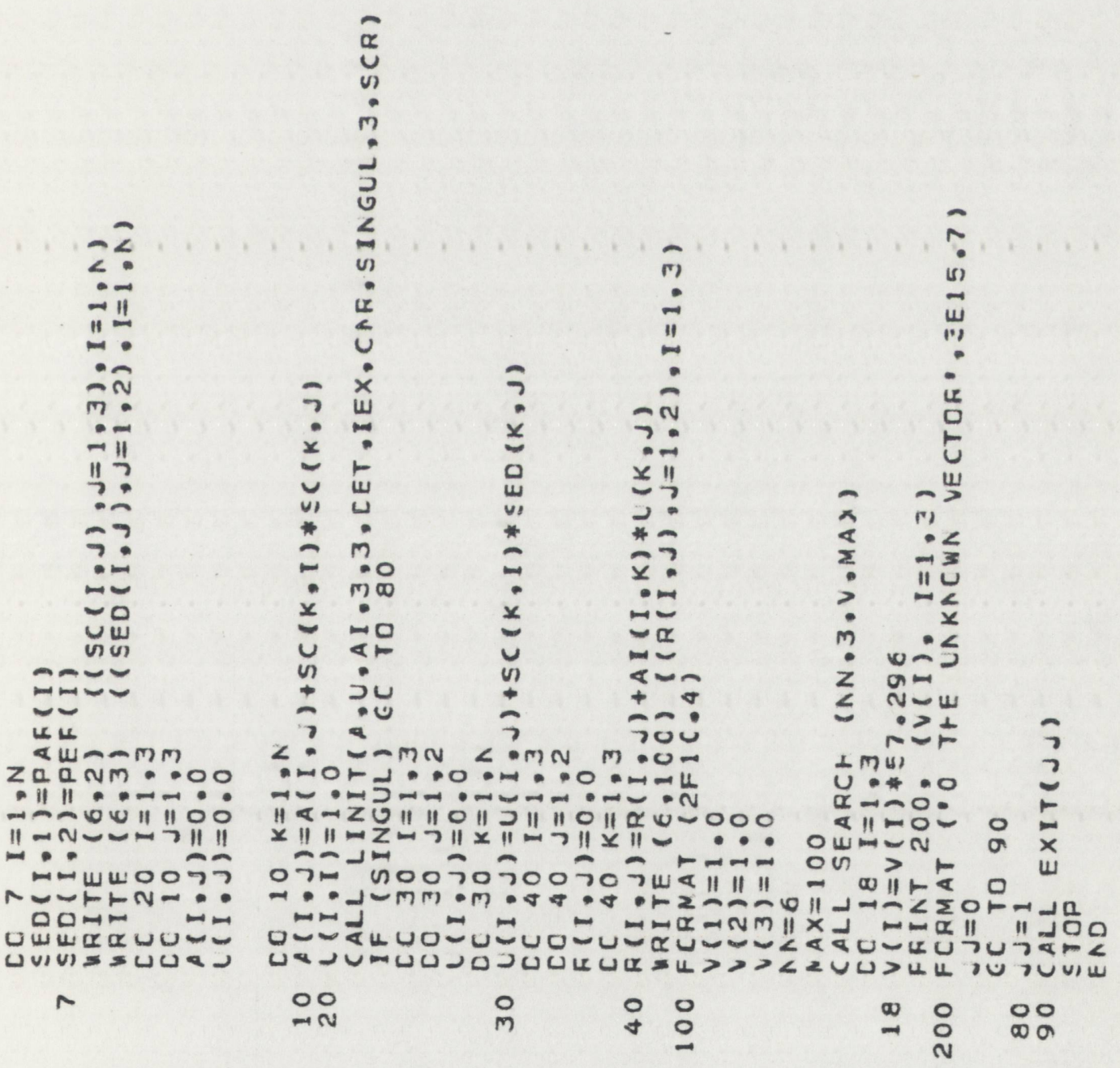




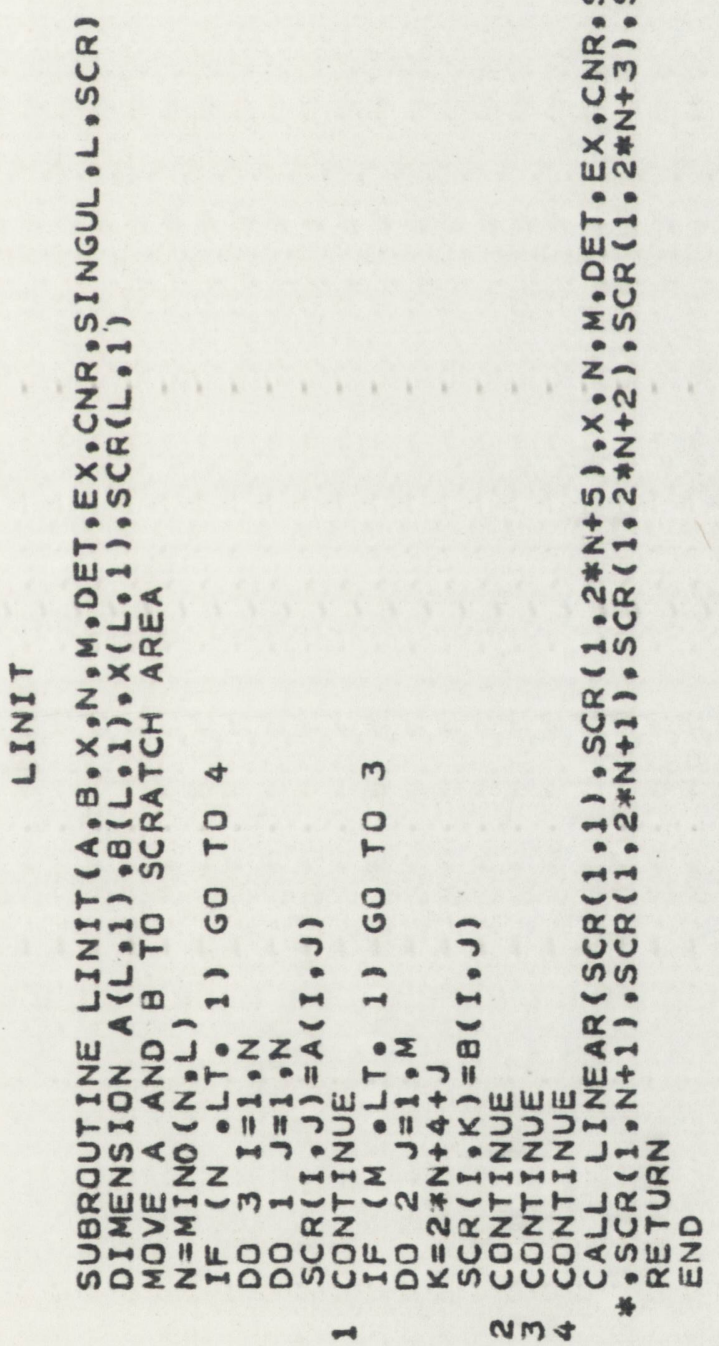

u

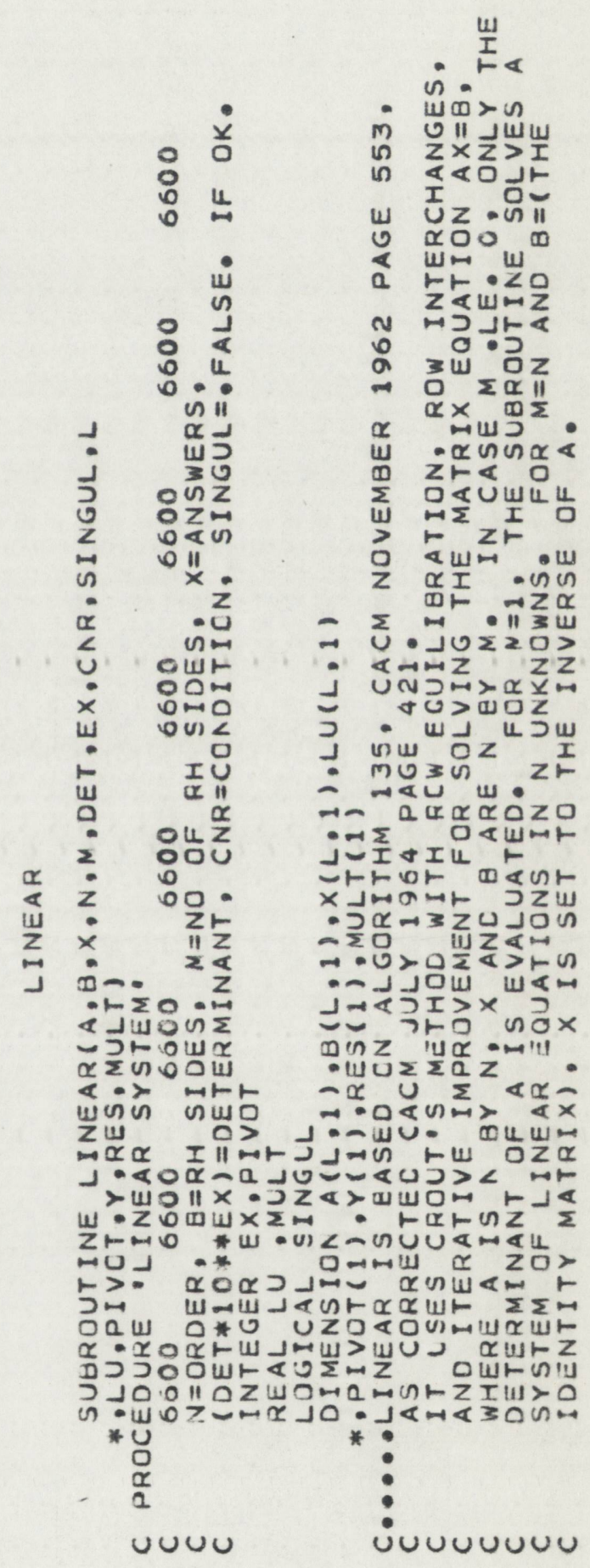




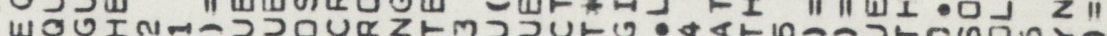

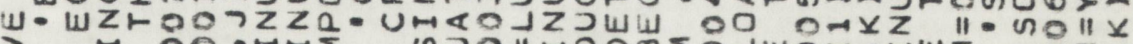
> जी

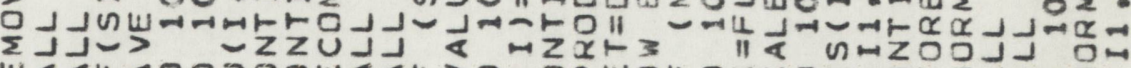

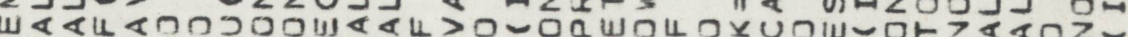

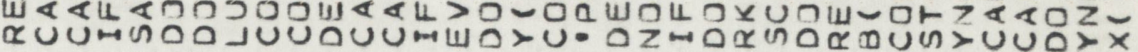

uv
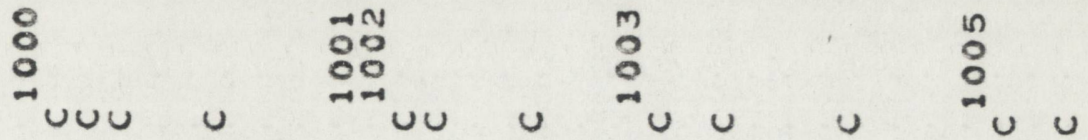


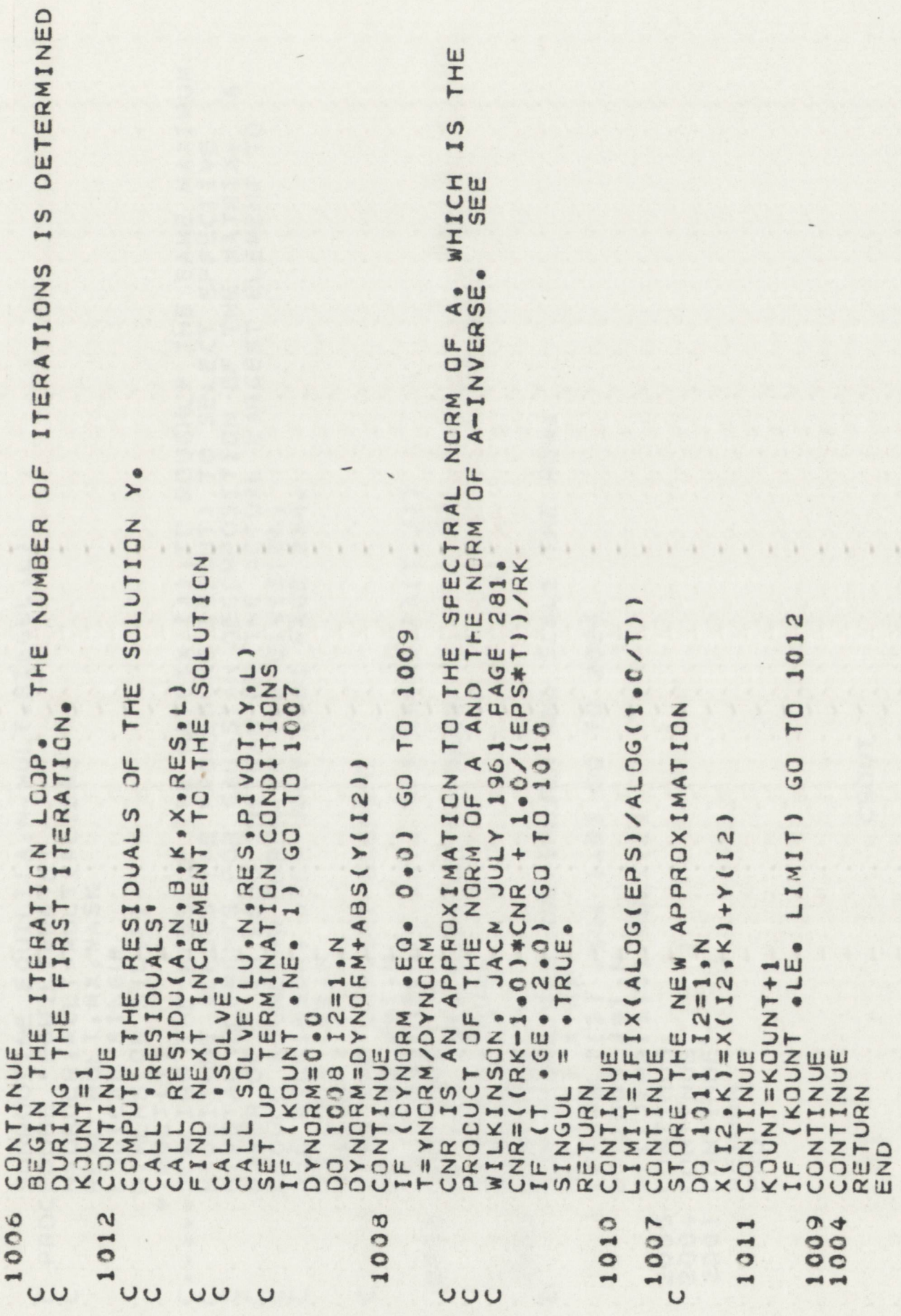




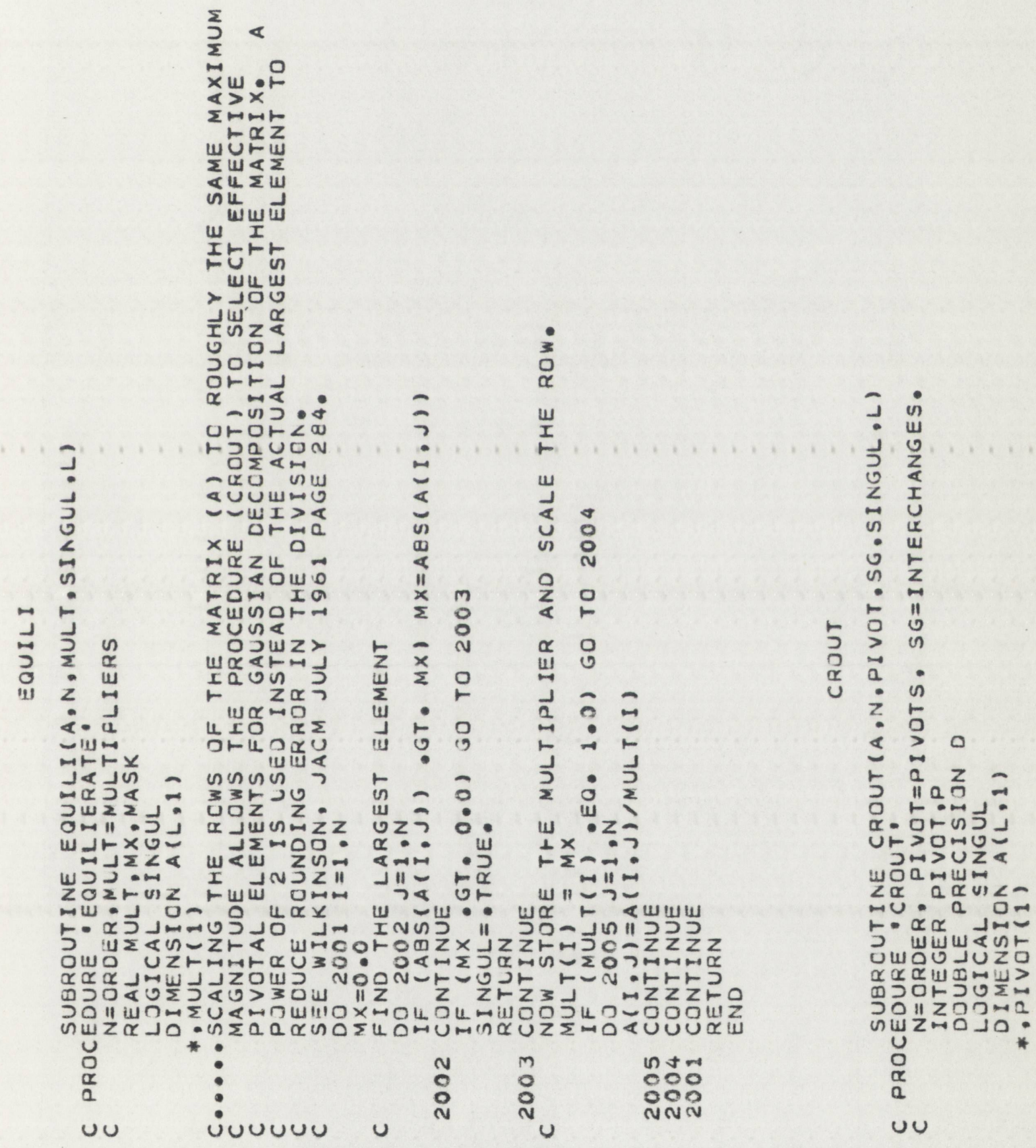


$\stackrel{\alpha}{0}$

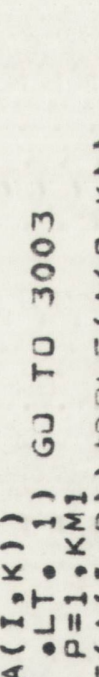

先

告

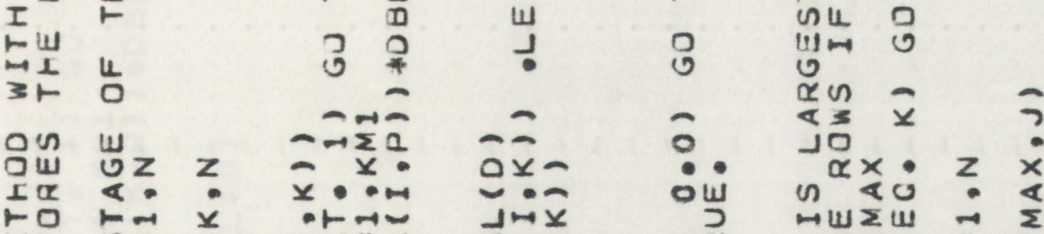

岾品 w

n- I-

งแ-1

$r \frac{2}{r}$

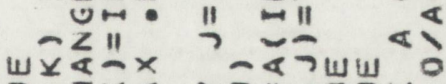

แ山ैं:

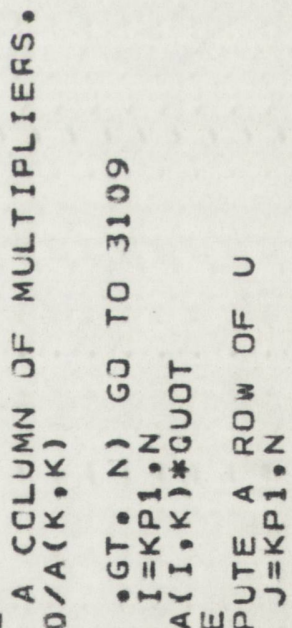

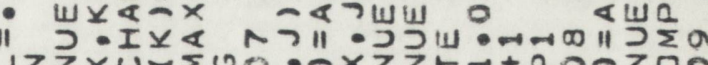

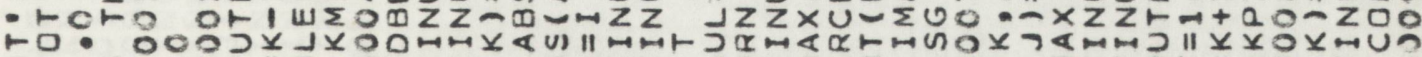

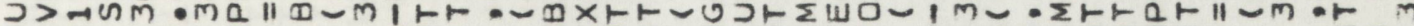
OM\|M

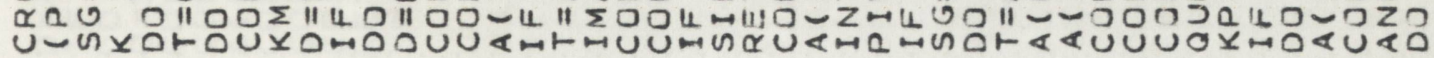

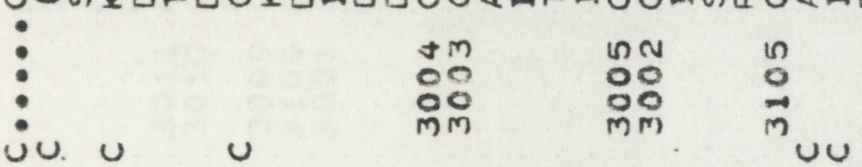

10
00
00
$m$ u

$\infty$
0
0
$m$ 

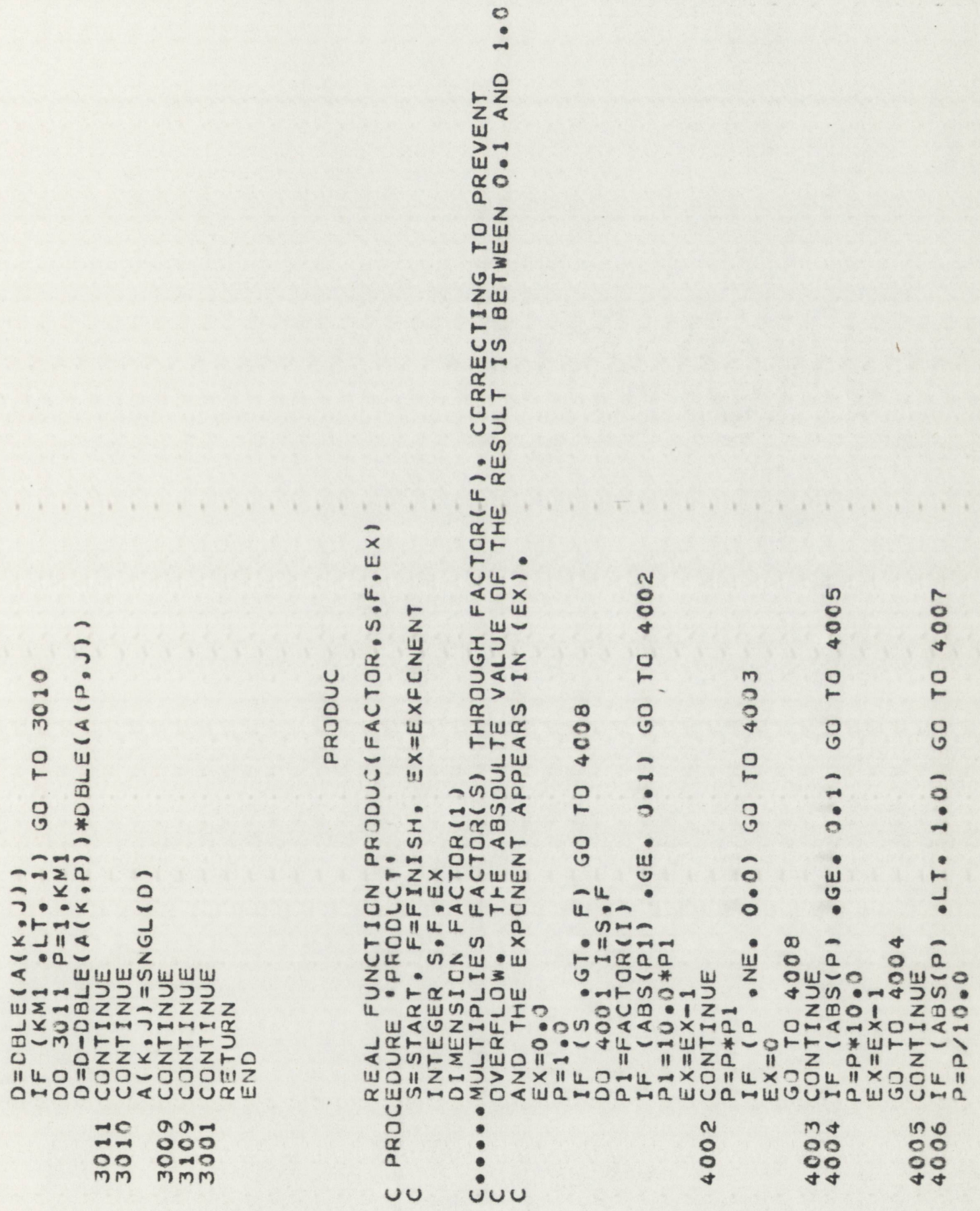
○

ㅃแำ

+十つつつUU

×0 $\sum_{m=1}$

山トトトト0

II zzzoro

$\times 0000 \alpha \omega z$

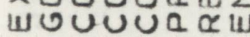

1.00
000
0.00
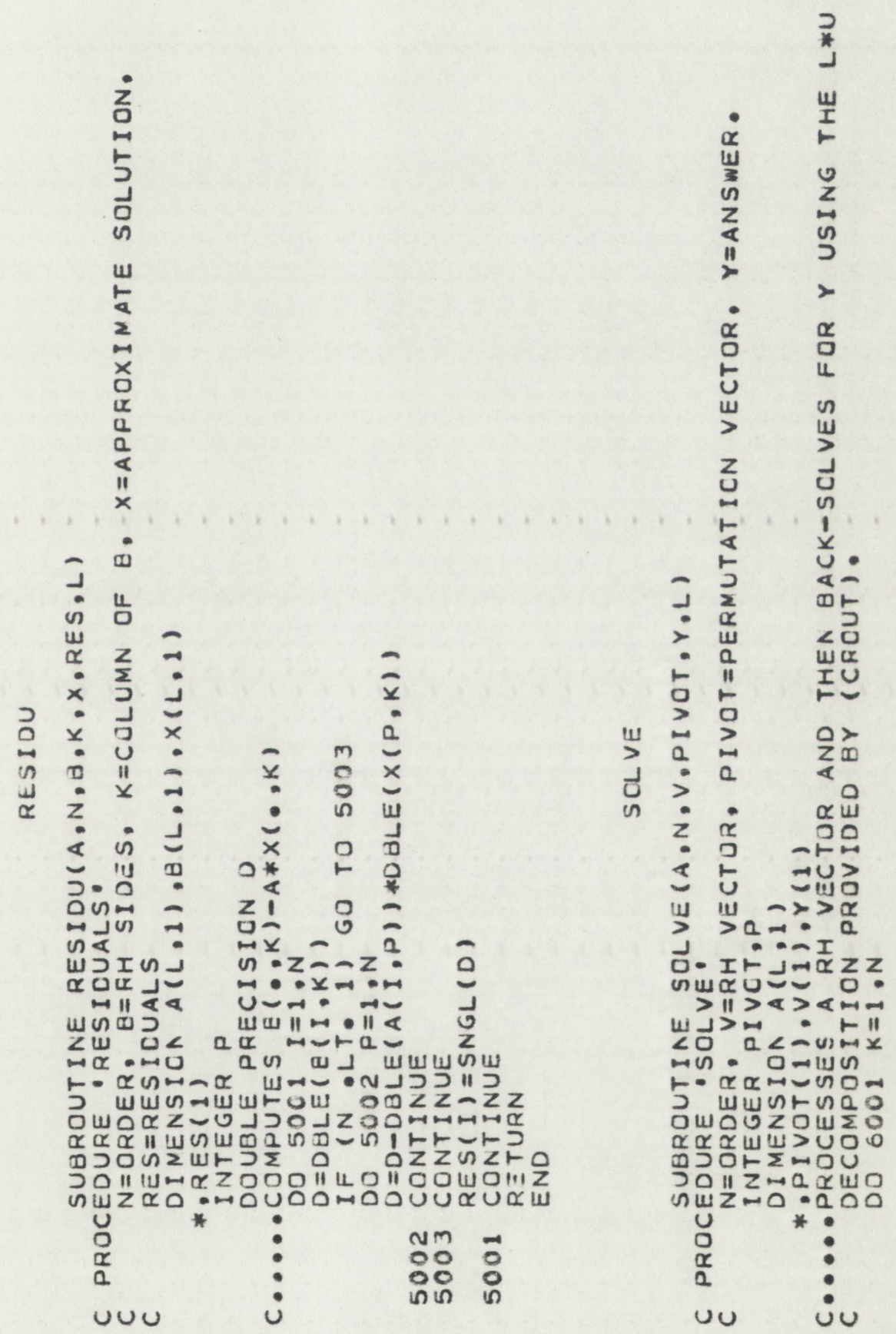


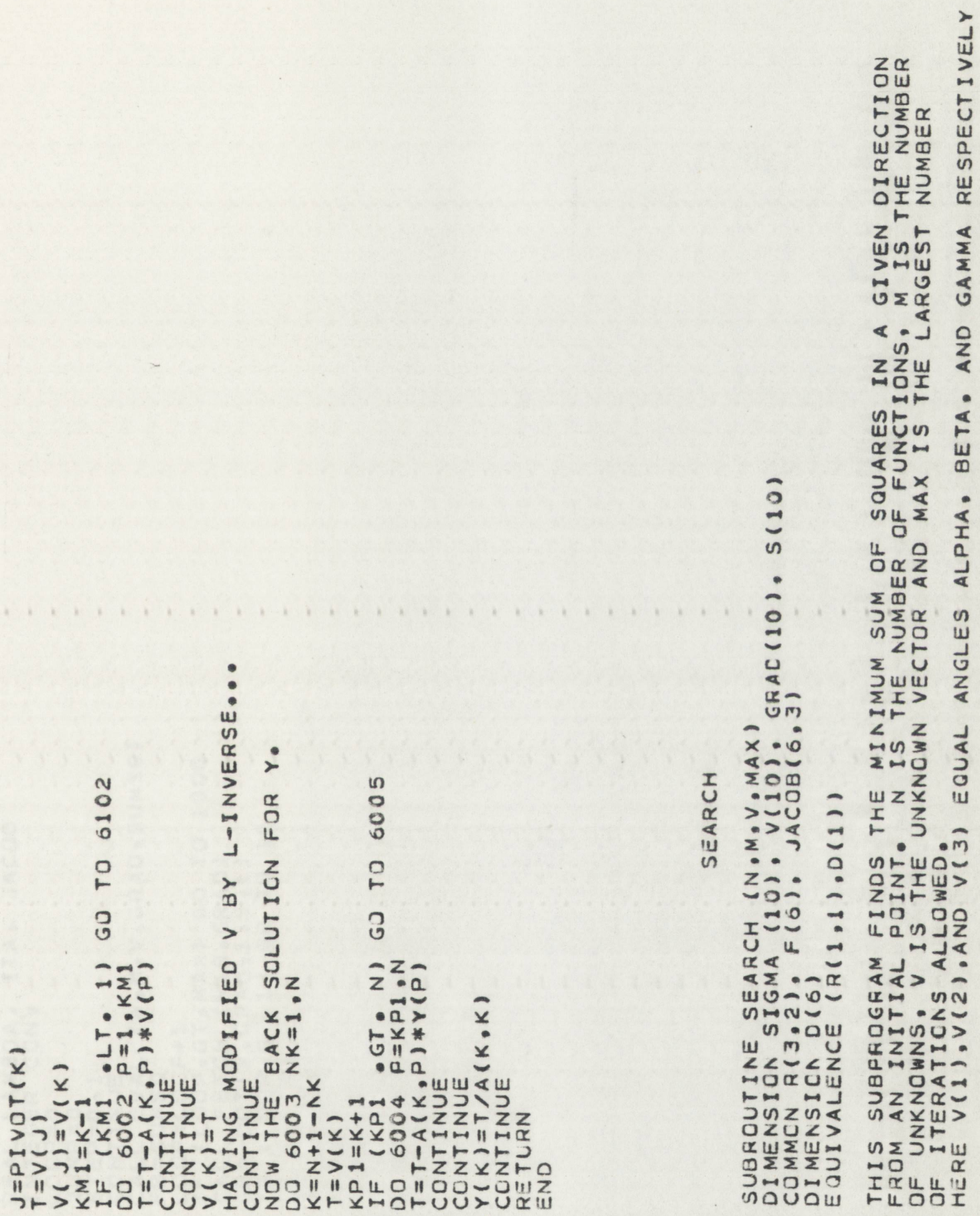




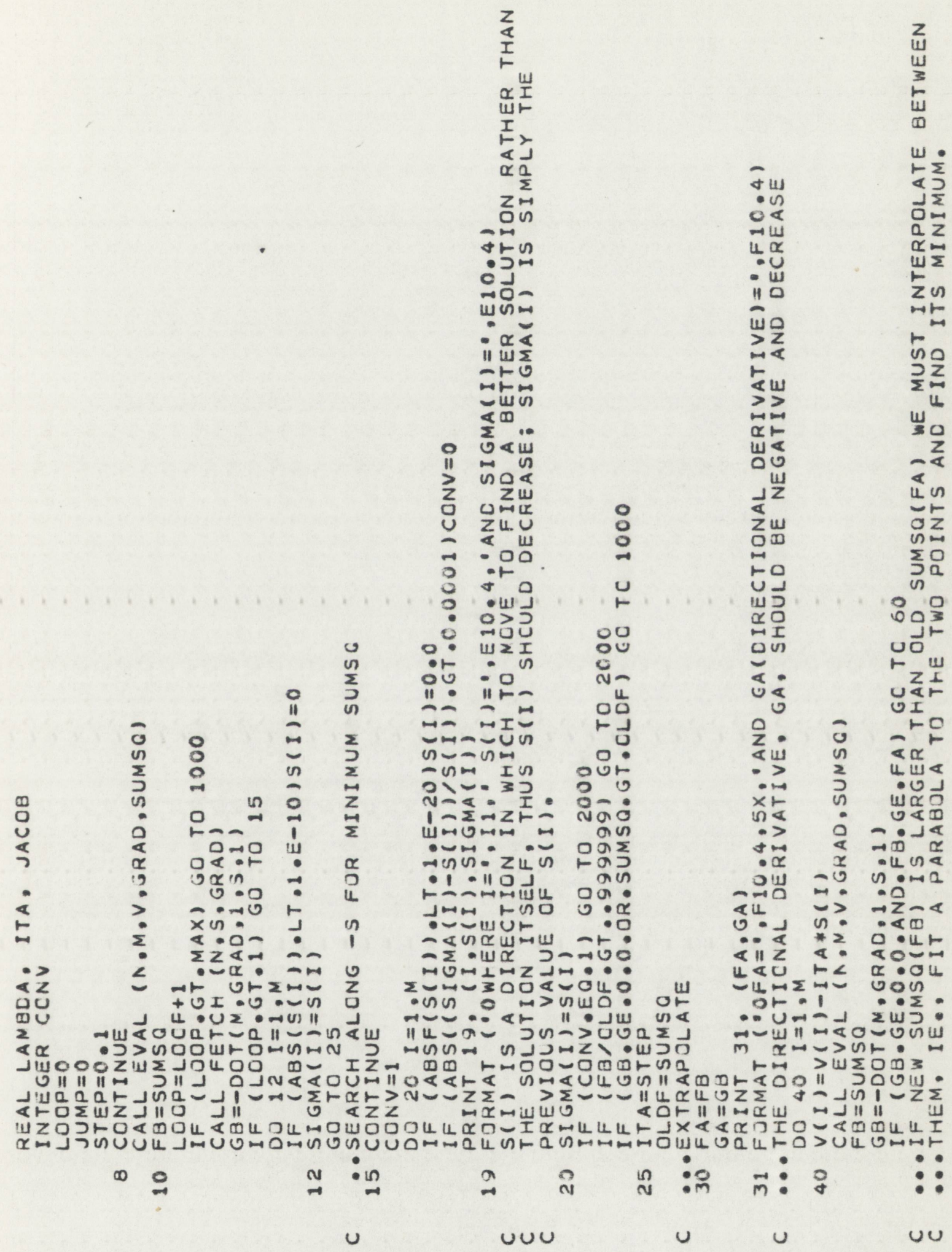




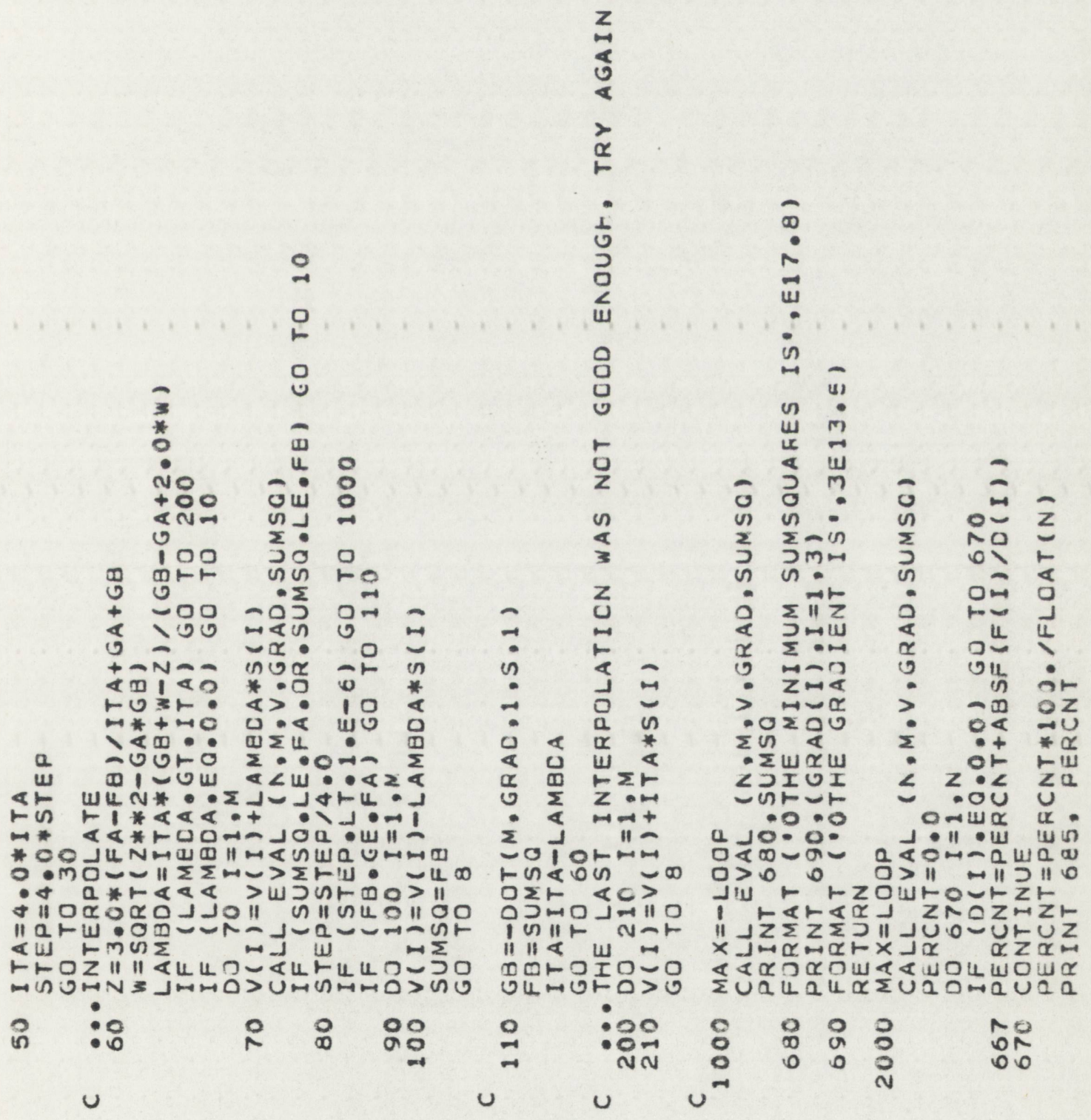



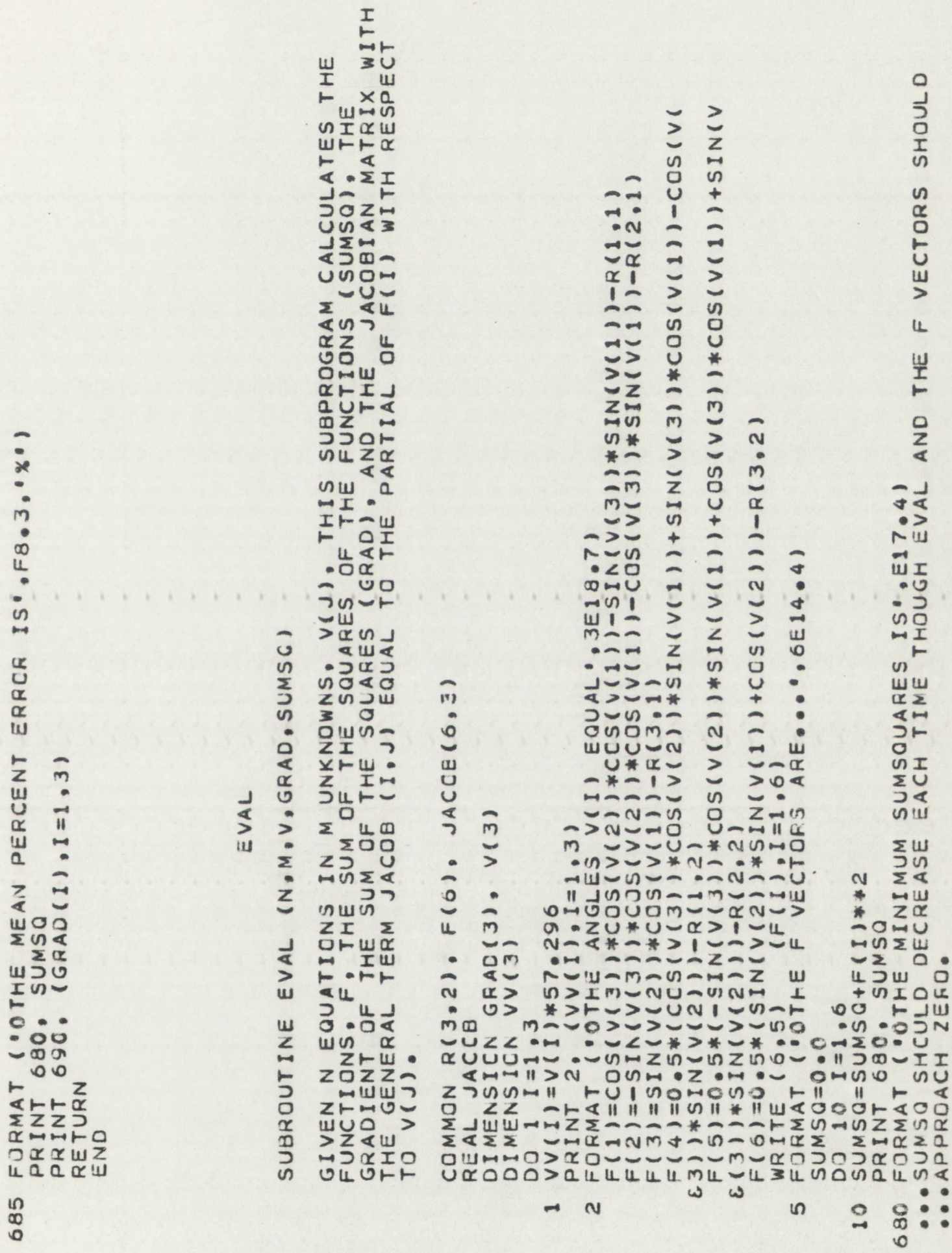

uuvunua 

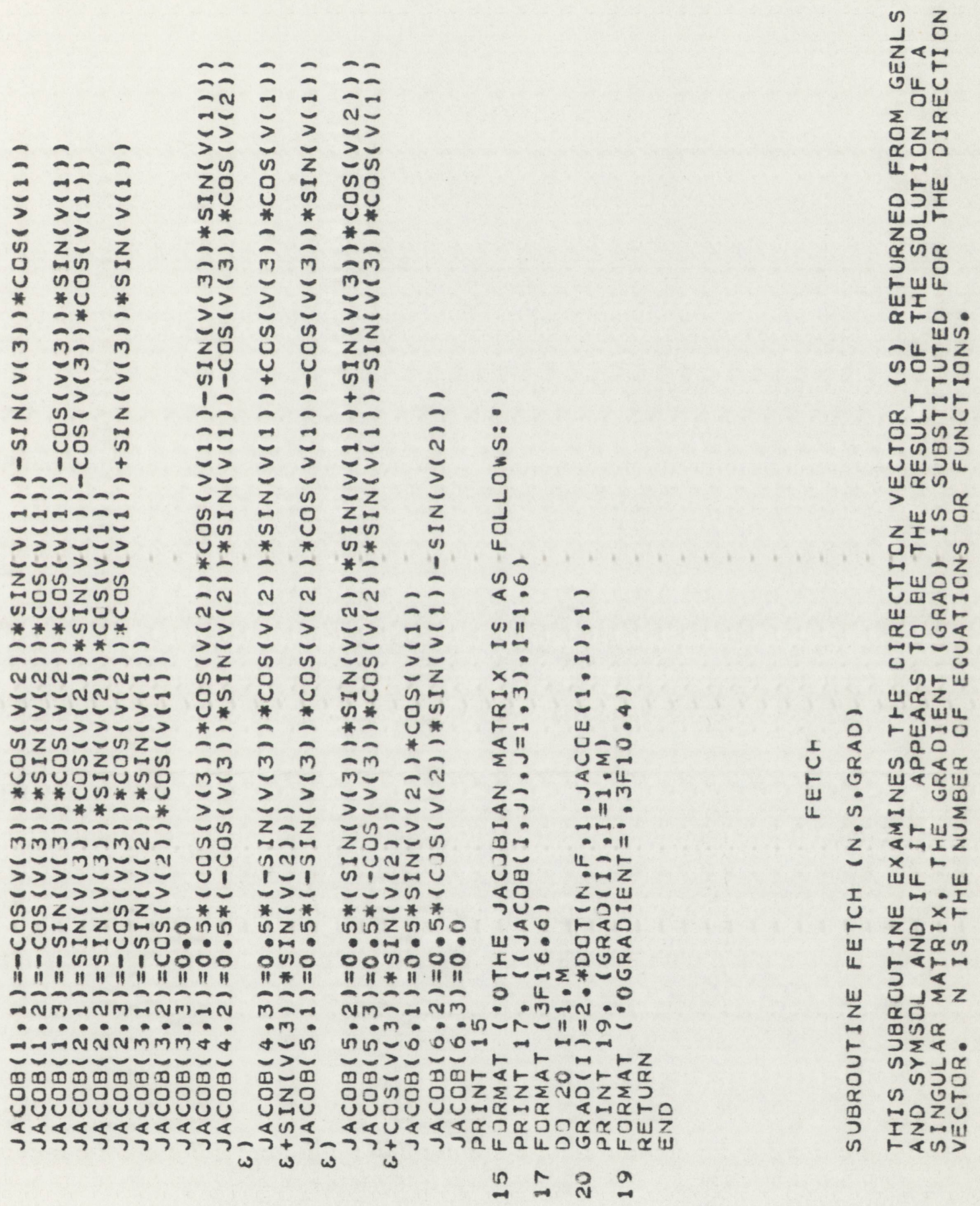

uvuuva 

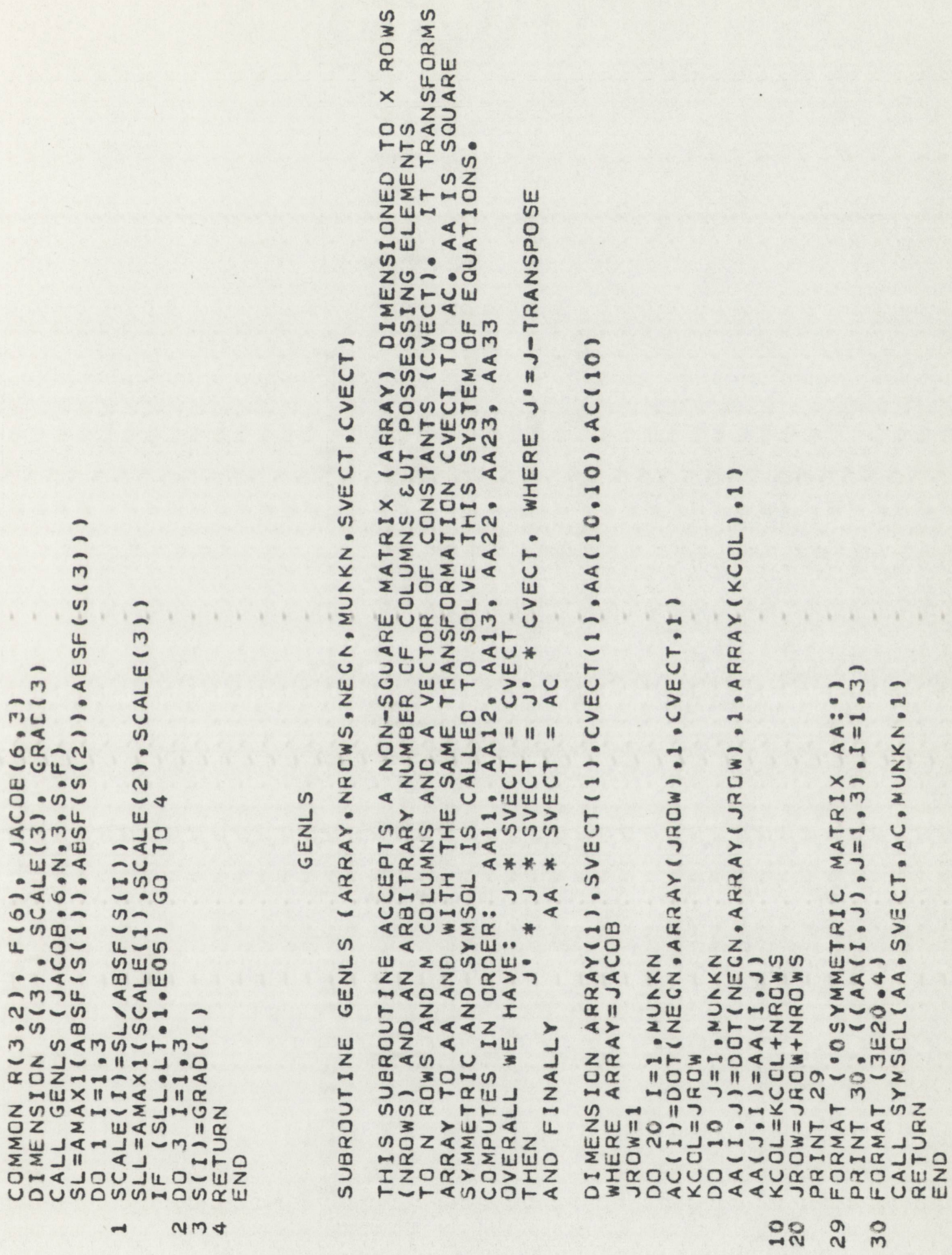

auvunumunas 


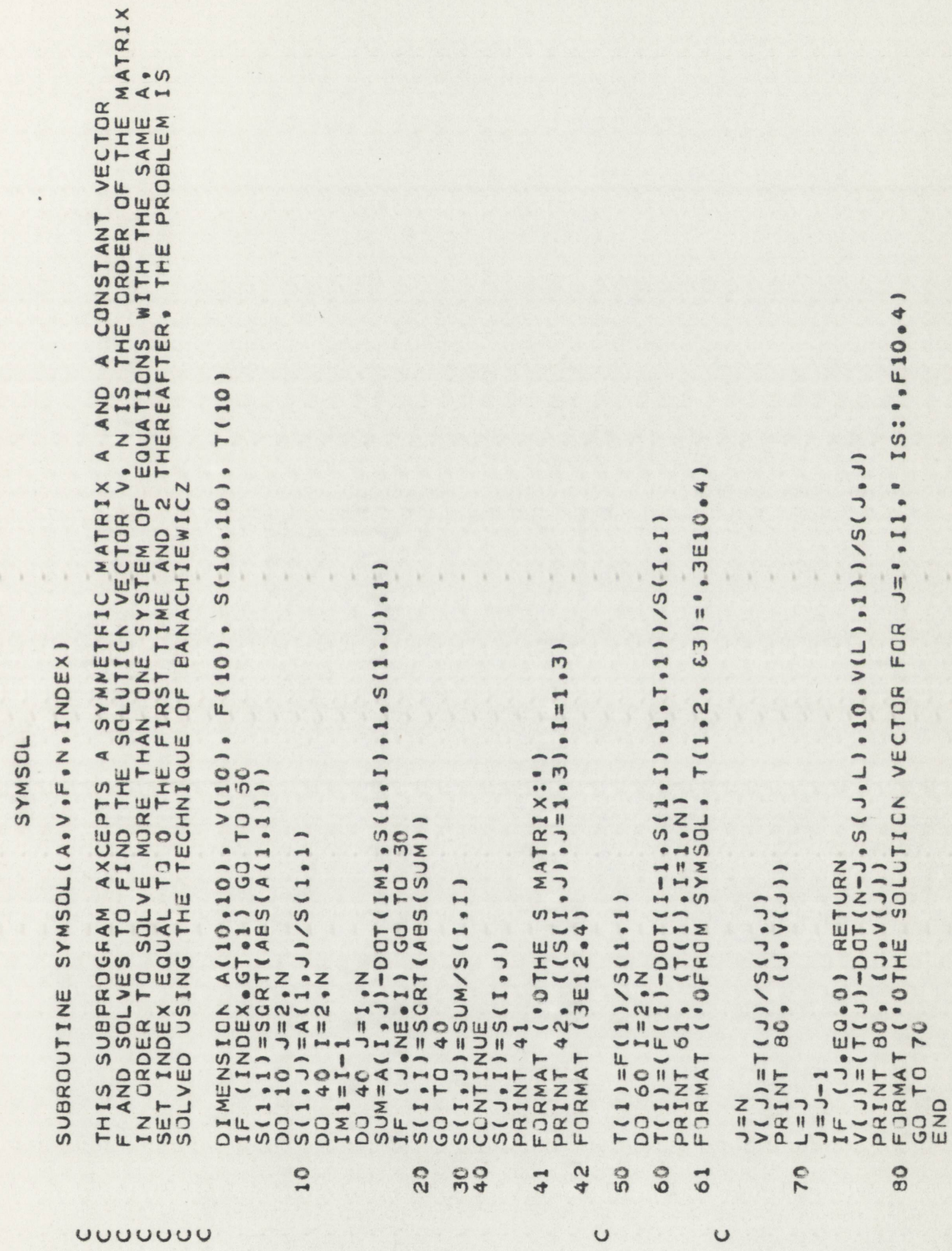




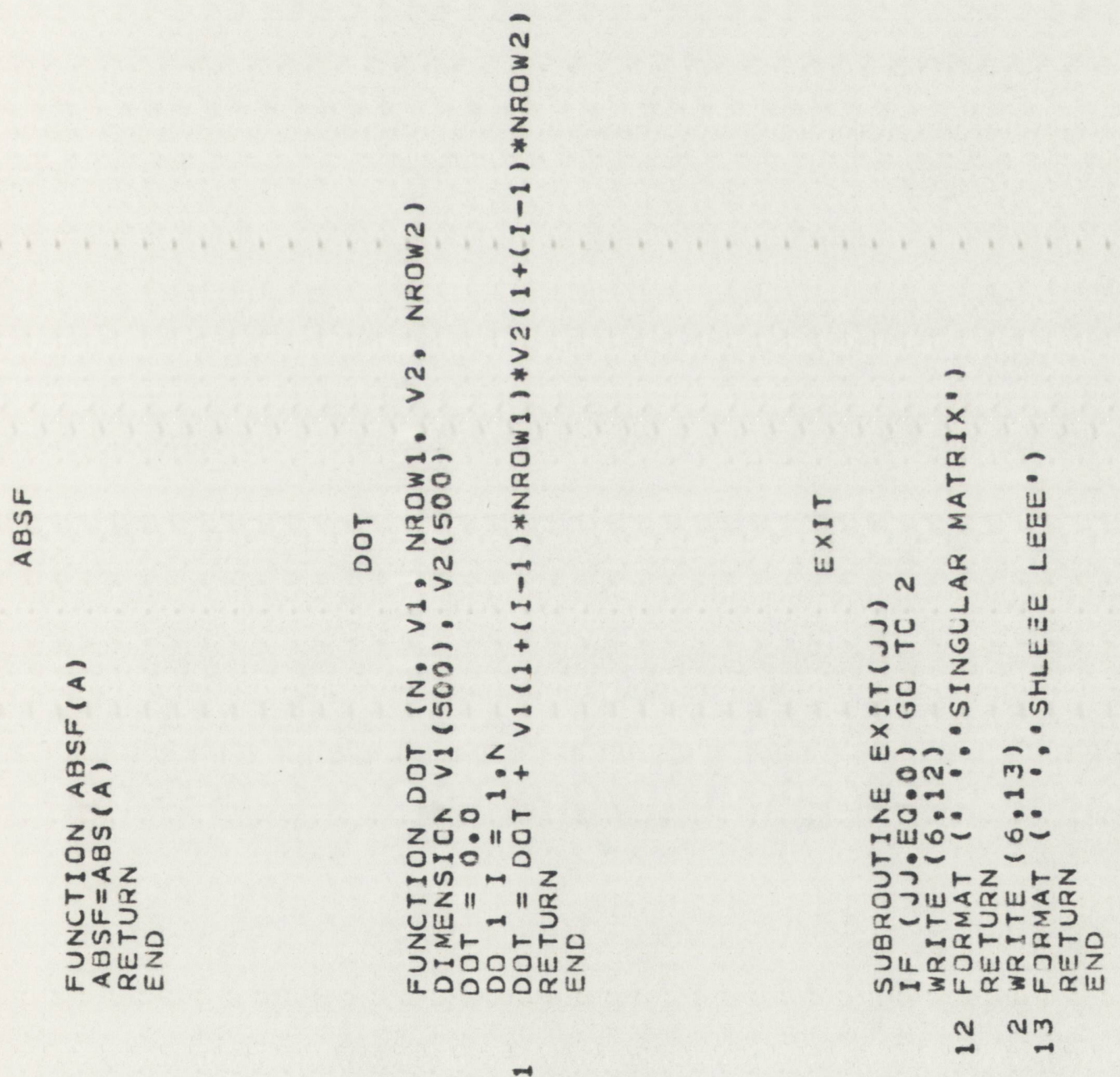




\section{Appendix B}

Degenerate of Main Program

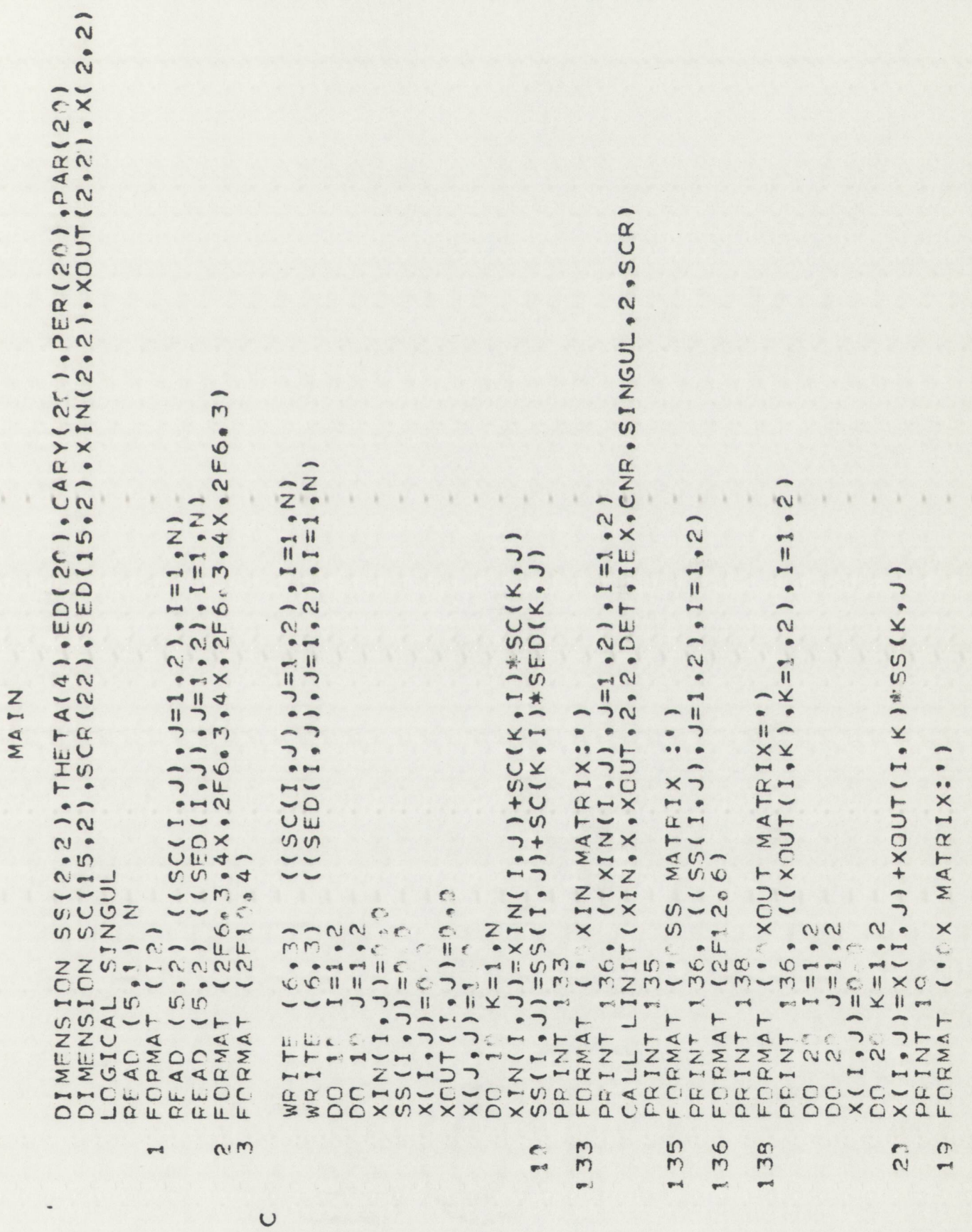




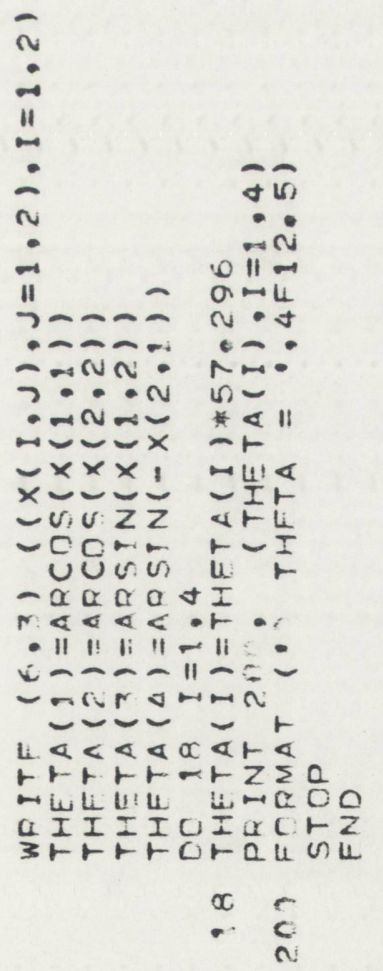




\section{Appendix C}

Main Program with SIMPLX

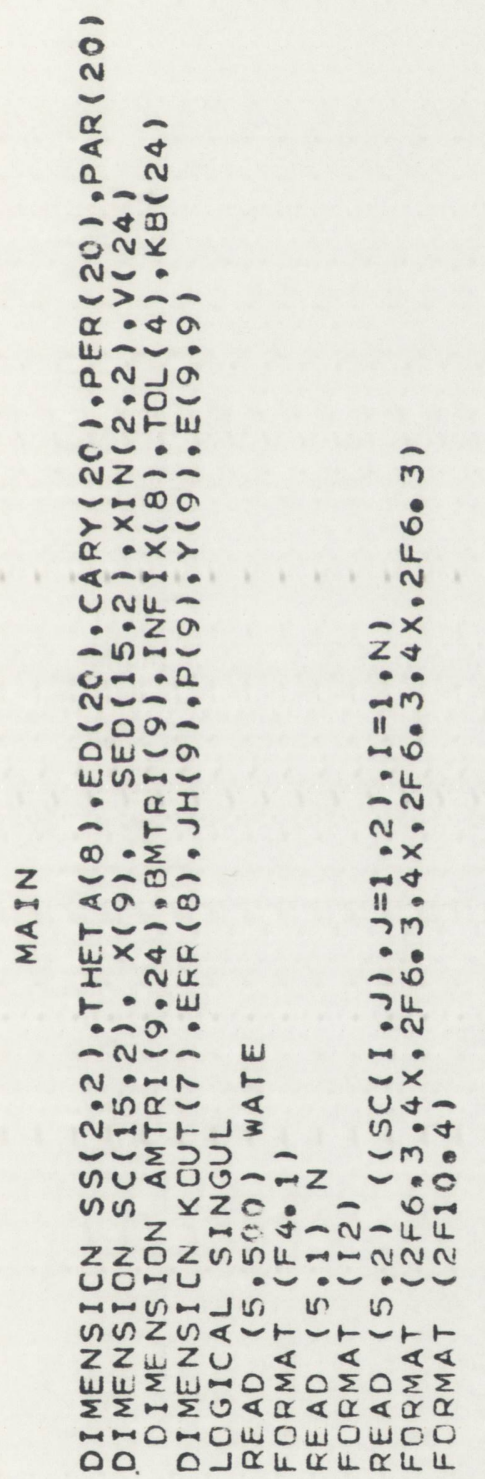

$$
\text { i } \approx N m
$$

$\frac{11}{1}$

茨

$\sum_{\text {in }}$

$\underset{⿱}{W}$

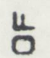

u

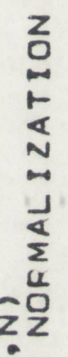

$\rightarrow \alpha$

iㅣ

$i n$

min

iw

$\propto u$

U⿺⿻一⿰冫⿰亅⿱丿丶丶⿱⿰㇒一乂

$-0 \mathrm{~N}$

嵌*

$\operatorname{an}^{*} \sim$

UI $\rightarrow * 0$

-Zン $\alpha+$

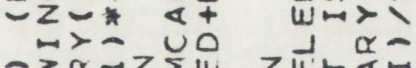

$\alpha=$

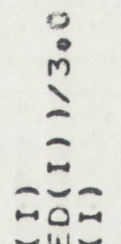

N N N N

年

인

$w$

$\int_{0}^{x} \alpha$

n⿺

uiv

F。

ชั

ด

山⿺辶

인

$>0$ 方

口 ए

nzơ

wvo -W0

$>1$ TNN NN

(1) $14 * * *$

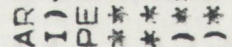

U一+ $+\pi=N$

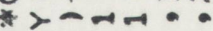

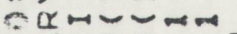

蓄菂命

$\operatorname{con}$

แேm

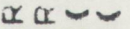

w 4 UU

a 0 电的

$++t$

山⿺辶寸

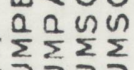

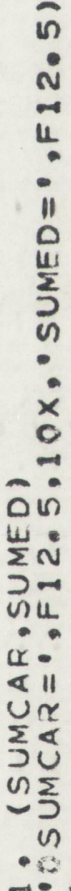

年

- juonúsuan ڤ」 | 一

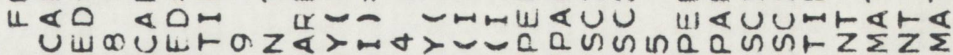

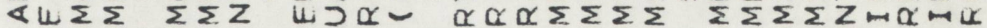

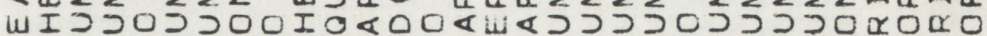

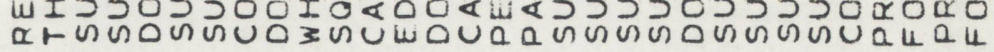

$\infty$

a

$+$

in $\vec{c}$

u $u$ 

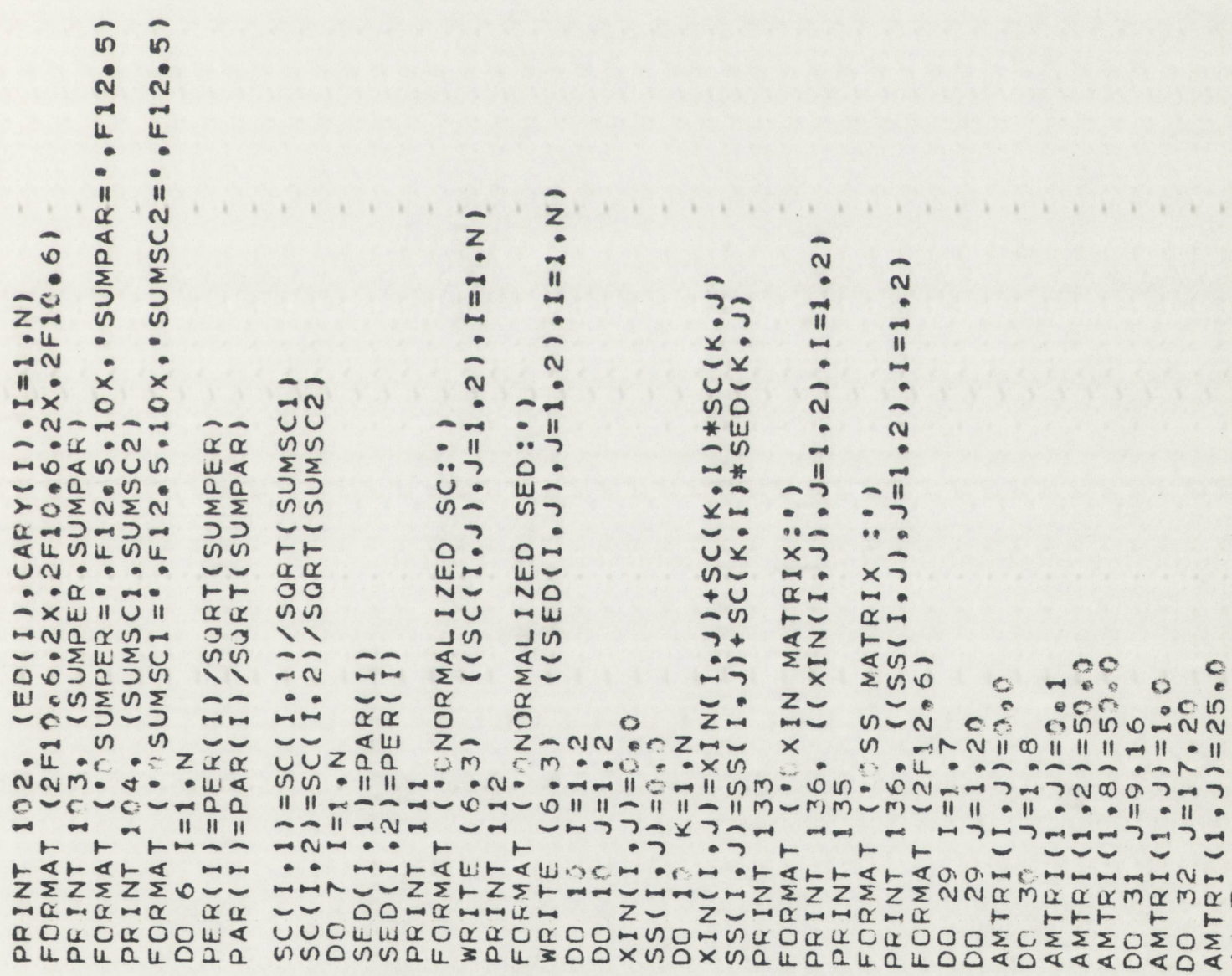
n $\times$. "1"

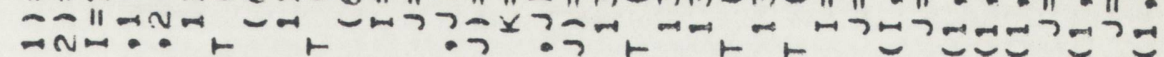
•. Mート

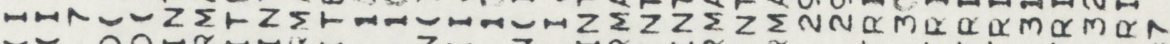

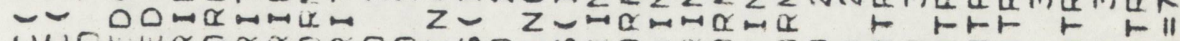

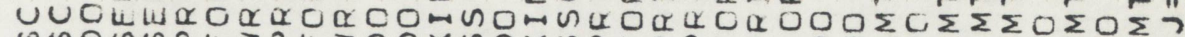

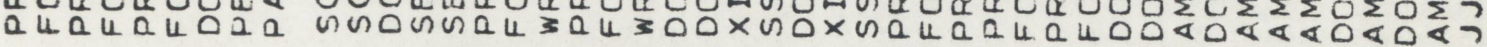
$\because \overbrace{0}^{2} e^{2}$

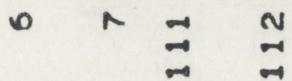
$\therefore \stackrel{m}{m} \stackrel{n}{m} \stackrel{a}{m} \hat{m}$
m $\tilde{m}$ 


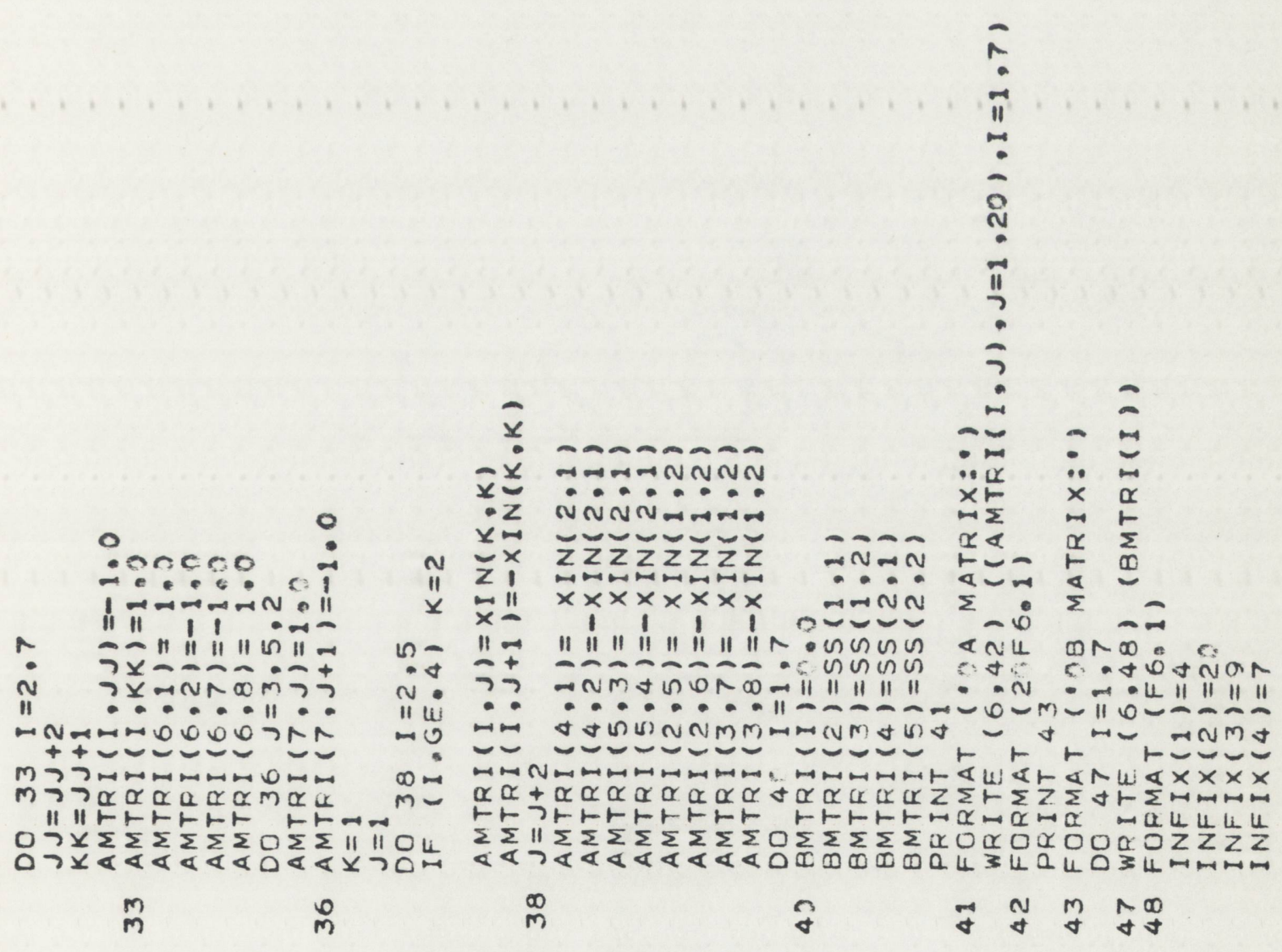




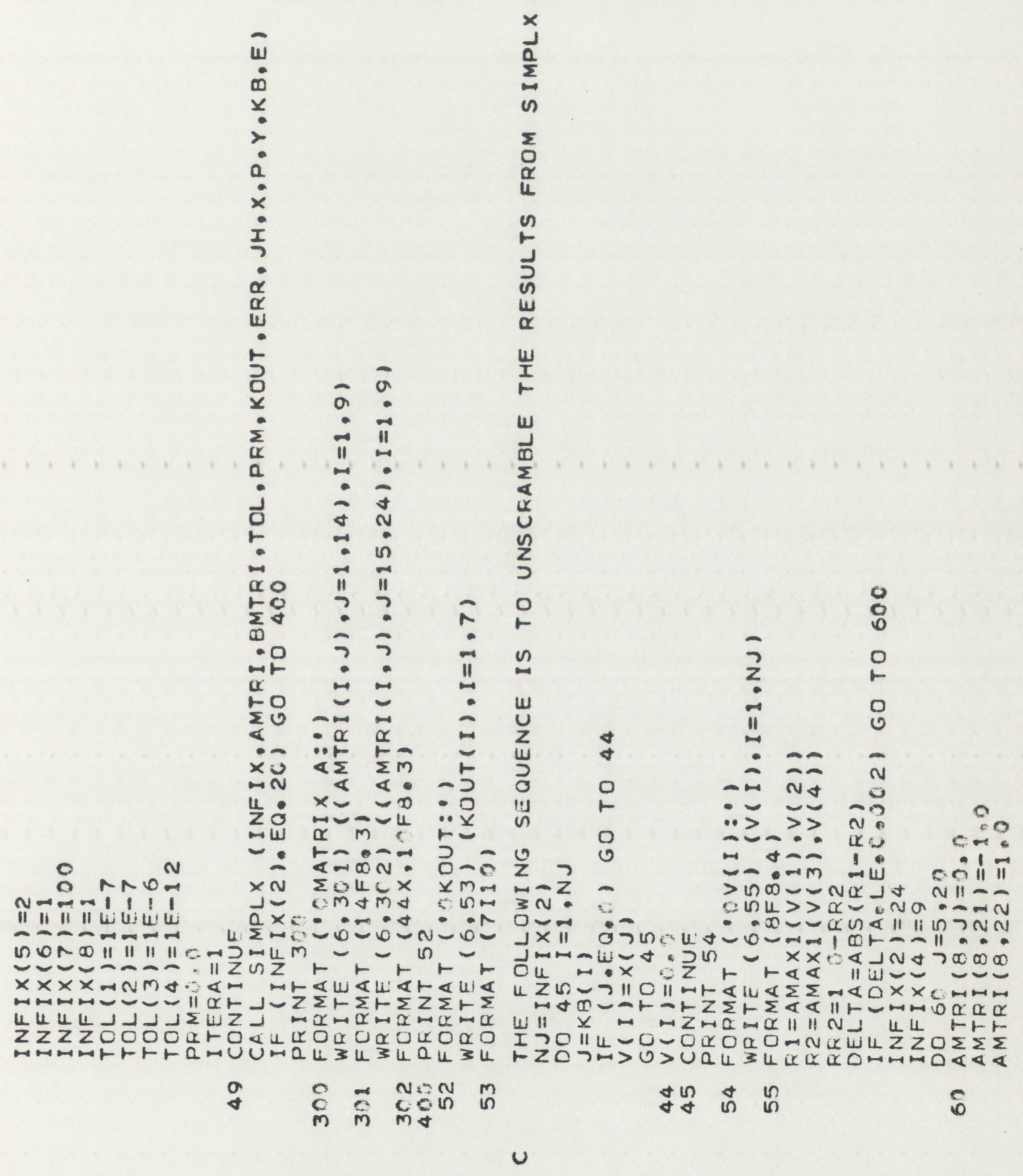




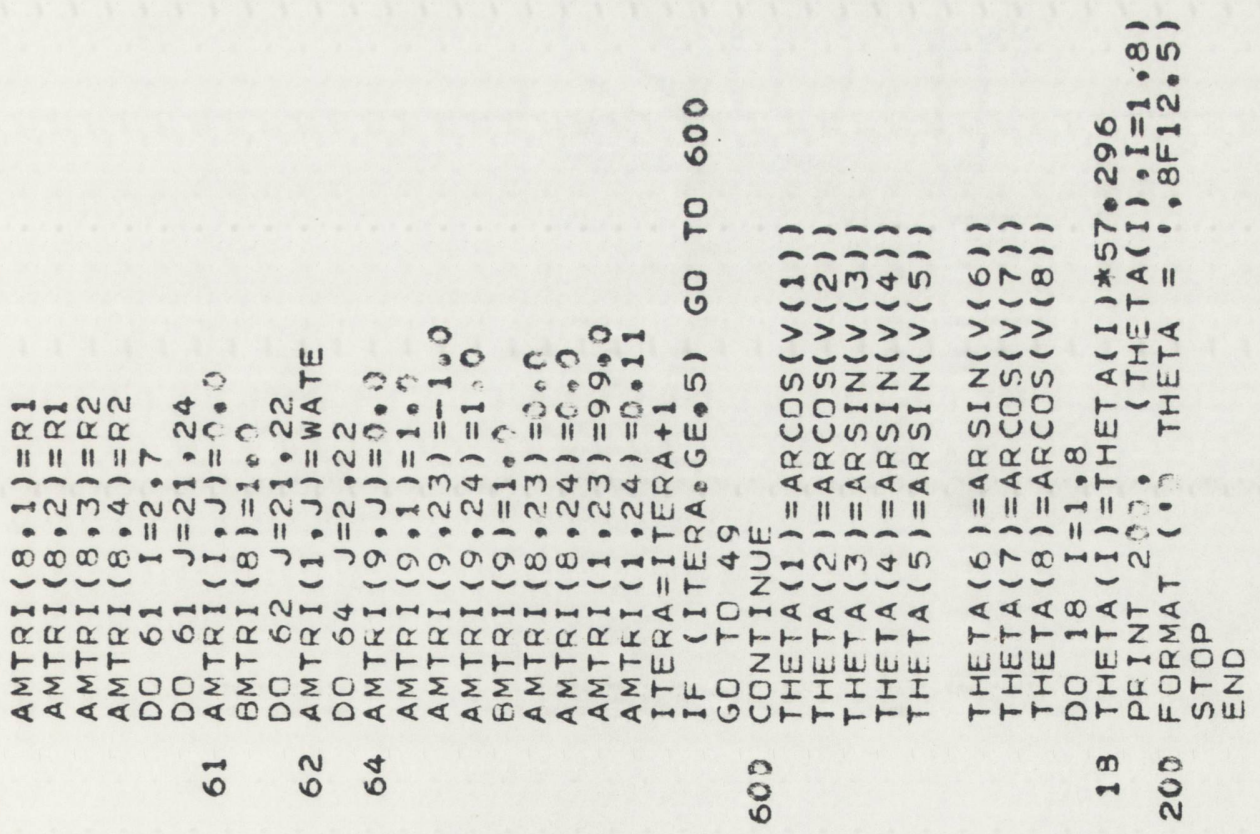




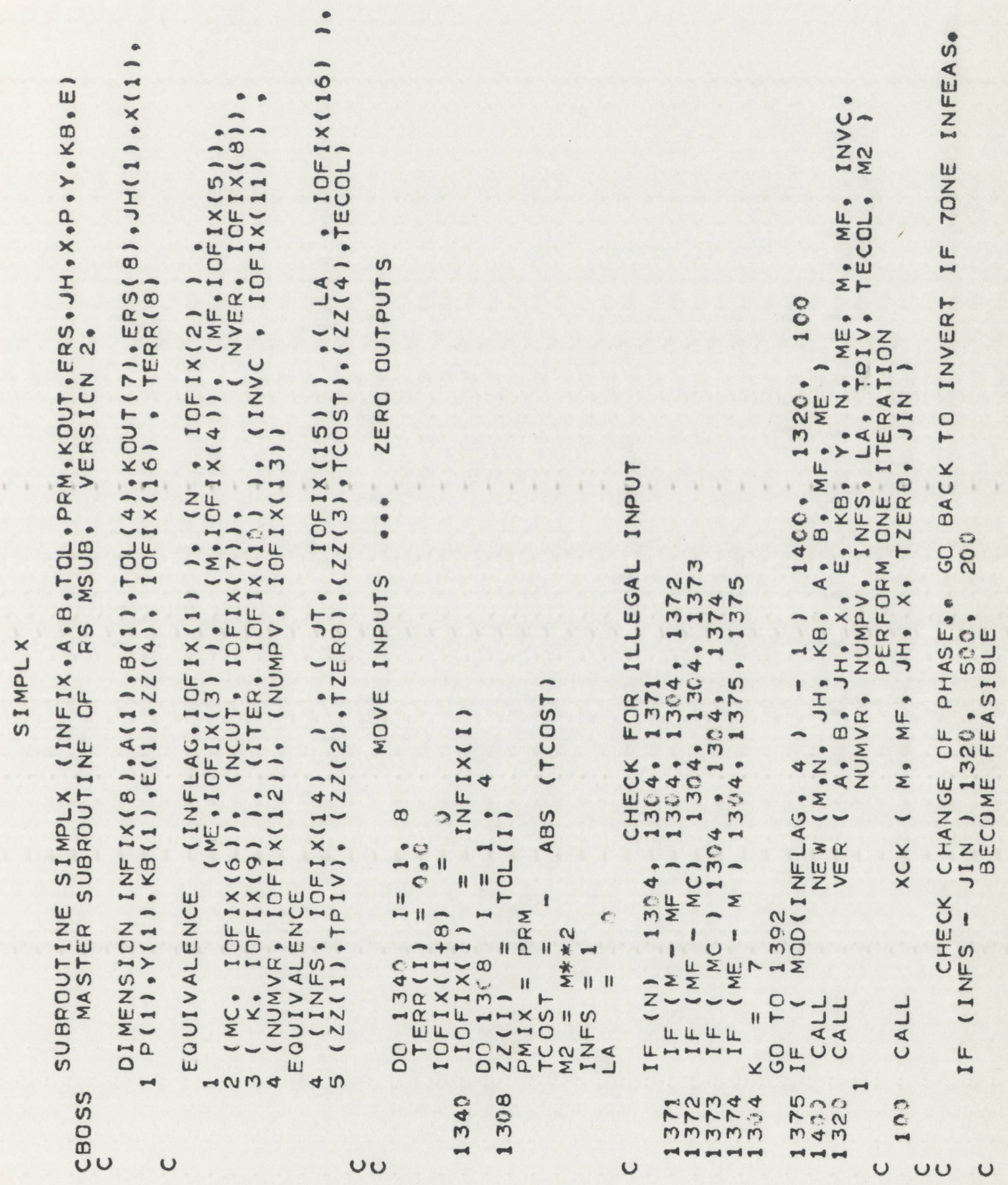




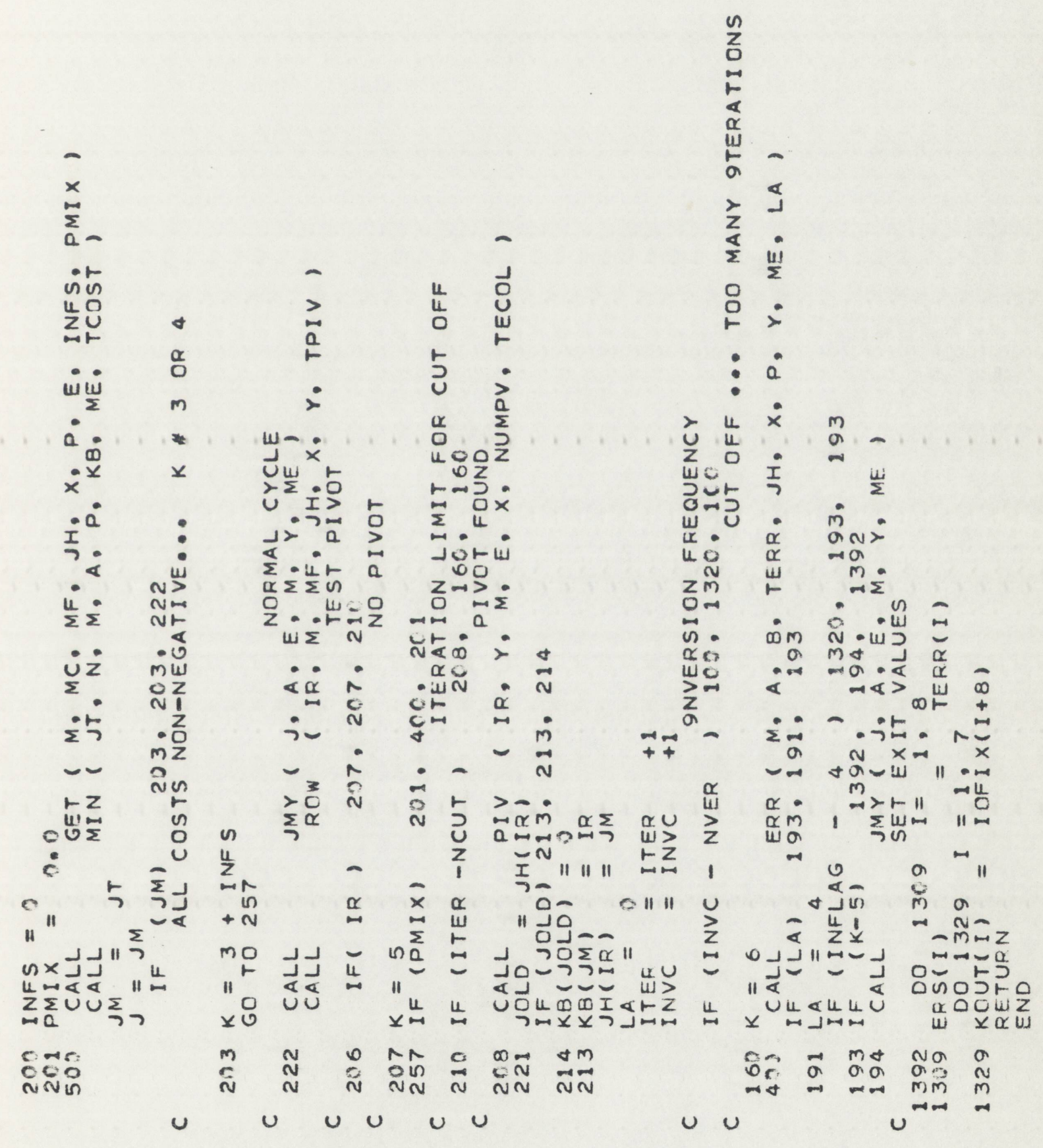




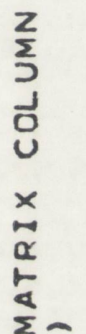

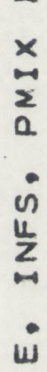

w

a

崖㐫

$\dot{x}$

$\frac{x=}{x}$

$\dot{i}=$

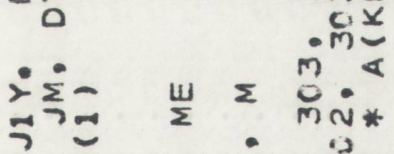

la $a$ * $\dot{0}$

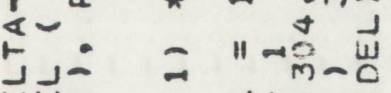

फ़न

\& 1 س

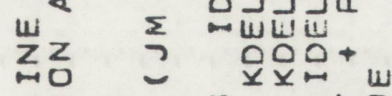

与n :

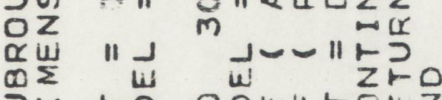

งัน

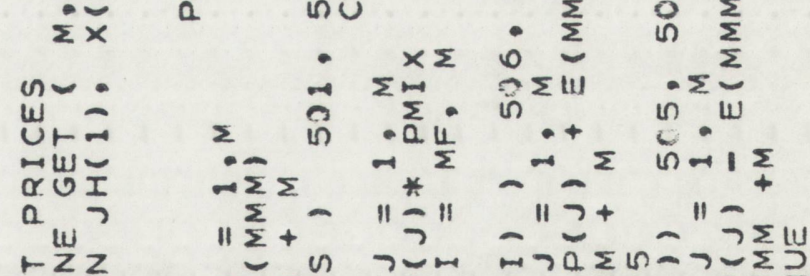

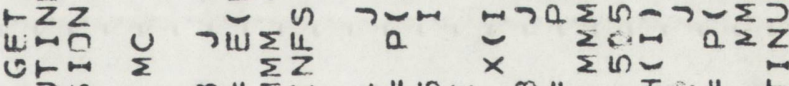

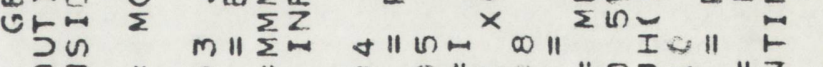

제 "

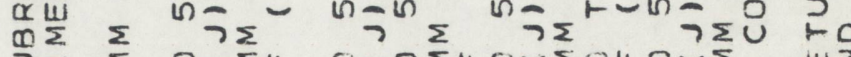

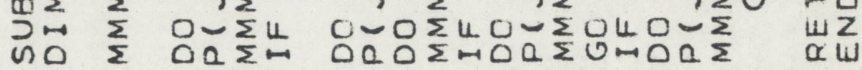

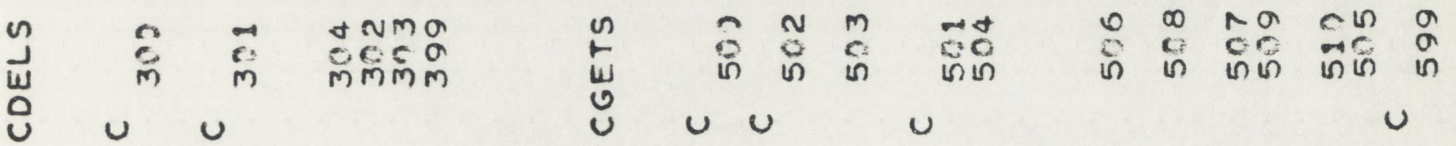




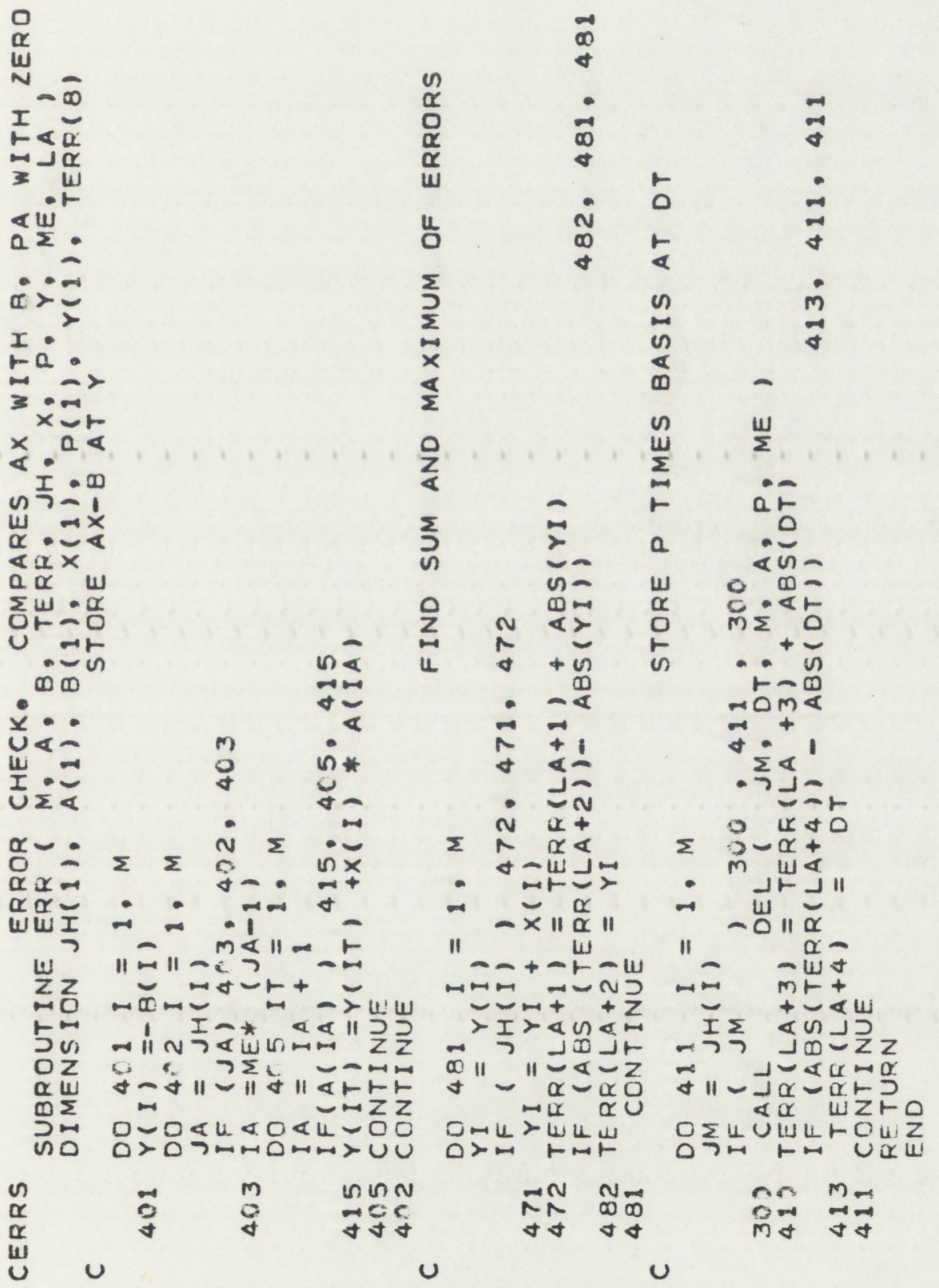




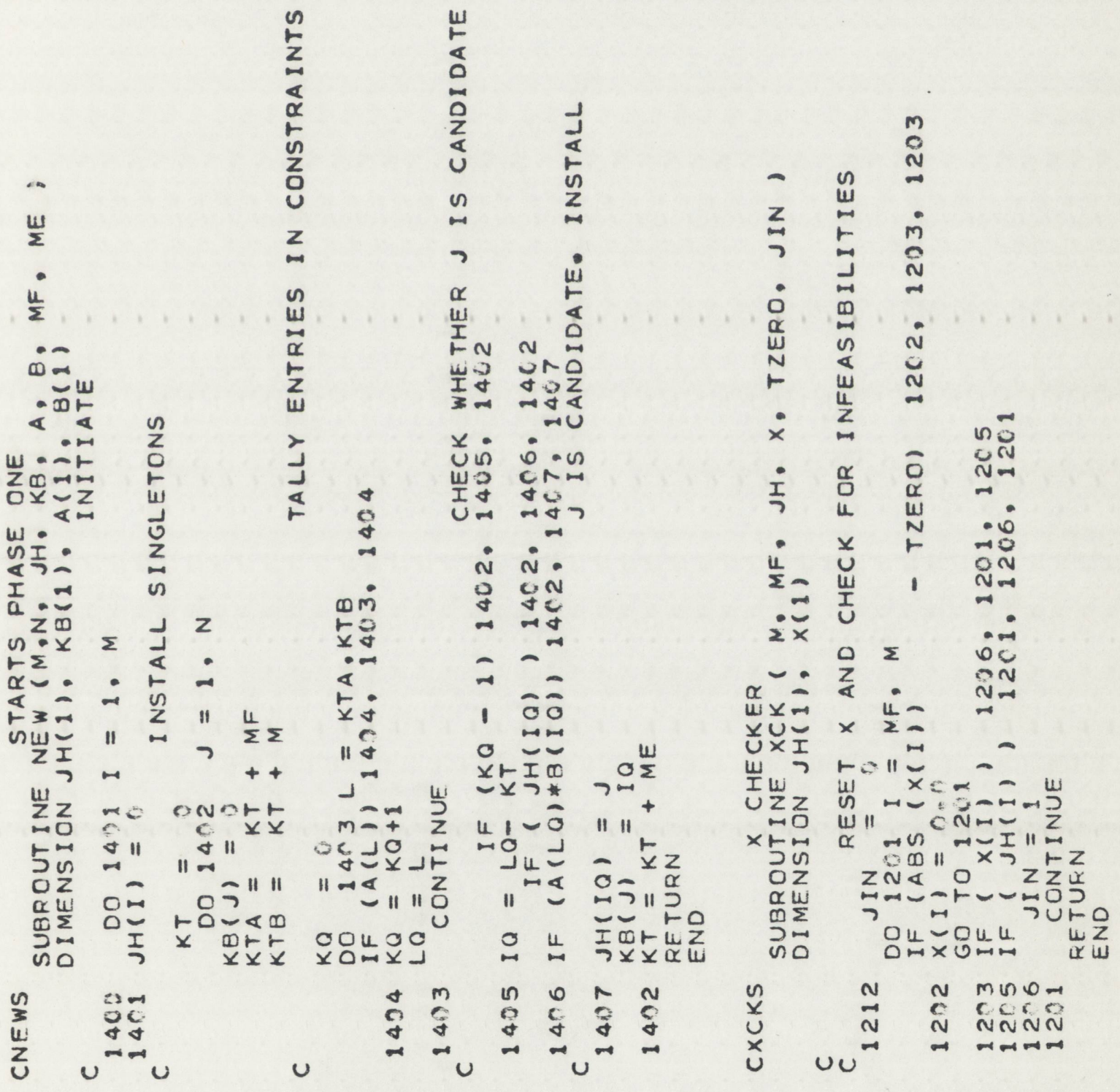




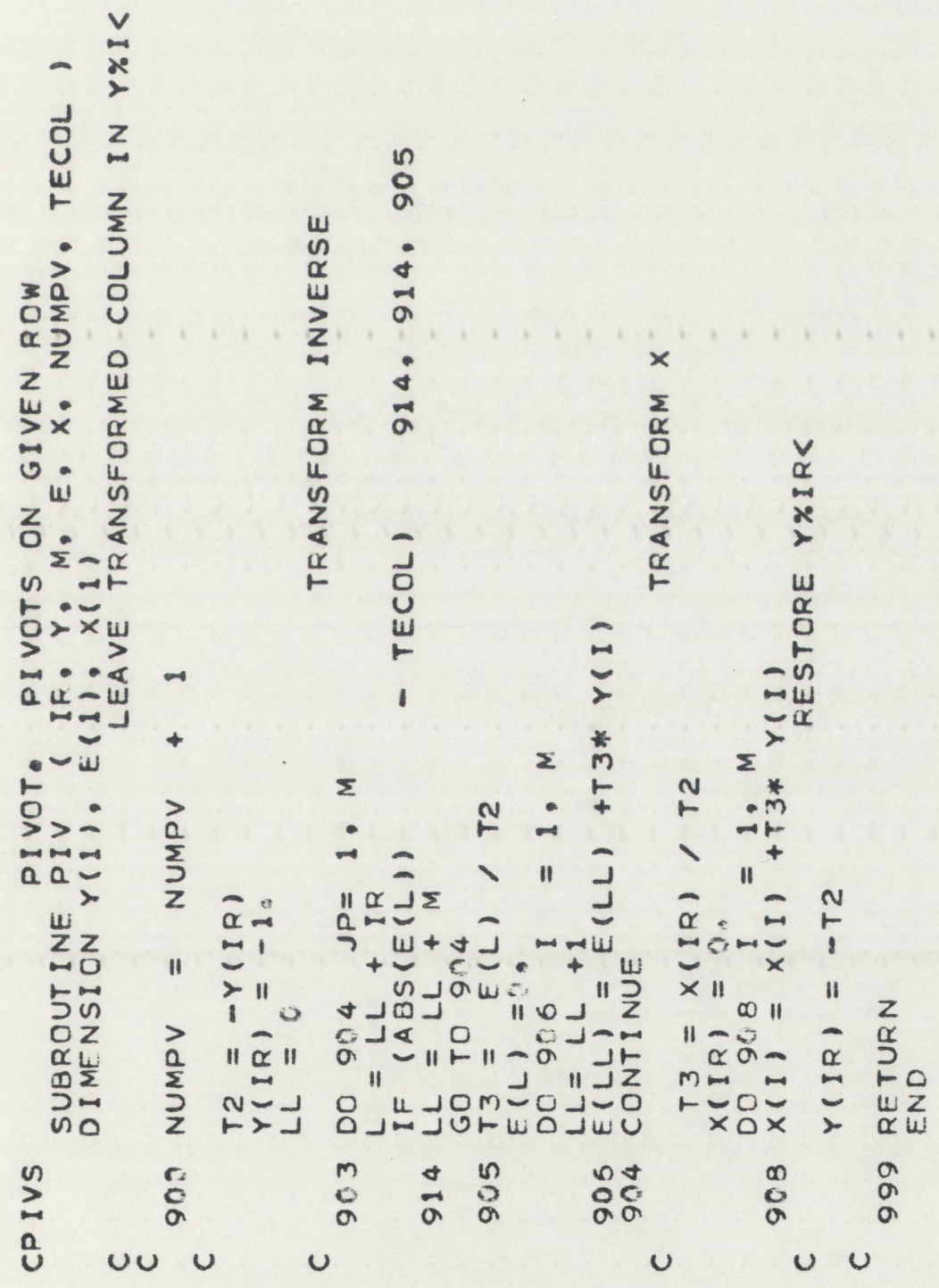




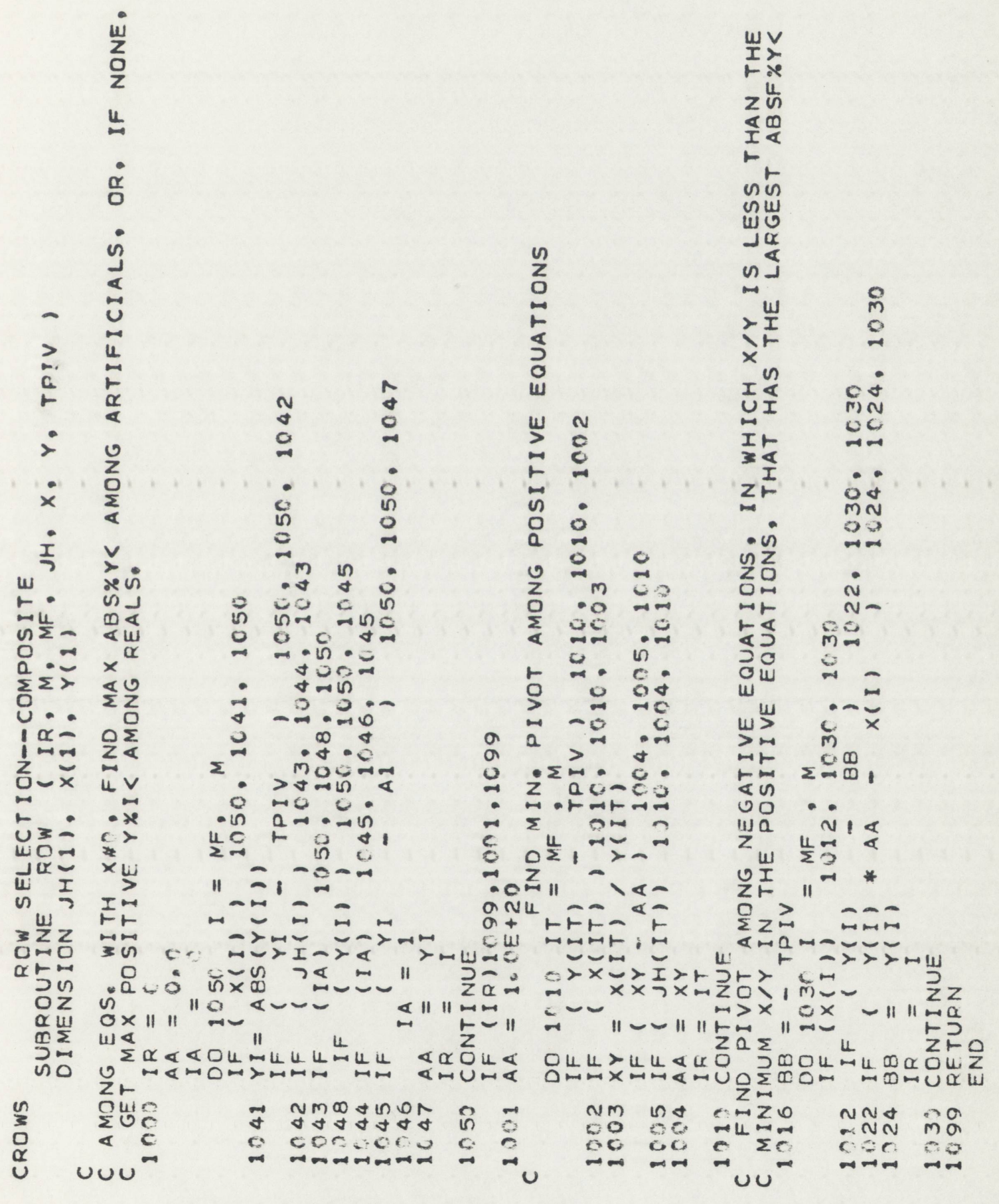




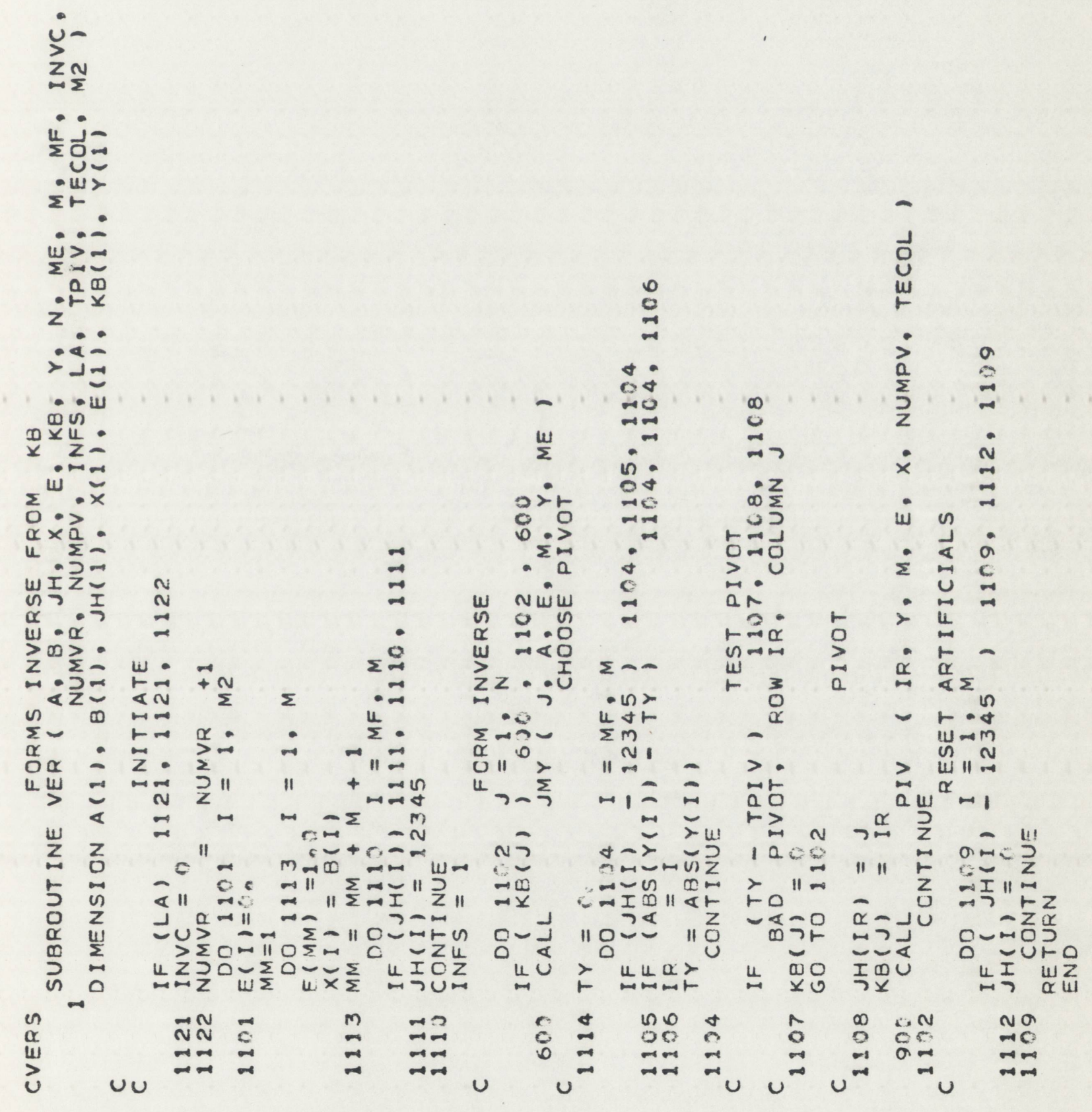




\section{Appendix D …SIGNAL}

The output signals from the photomultiplier tube obtained on the oscilloscope must be converted to extinction coefficients eventually in order that they be compared to those of the crystal or film. The photomultiplier signal (S) equals the gain (G) of the tube times the intensity of light incident upon it. That is, for both polarizations,

$$
S_{\|}^{0}=G_{1} I_{\|}^{0} \quad \text { and } \quad S_{\perp}^{0}=G_{\perp} I_{\perp}^{0}
$$

where the superscript indicates field-off conditions; fieldon conditions will be denoted by primes.

Since

$$
\frac{\Delta \varepsilon}{\varepsilon}=\frac{9 R-3}{4}\left(3 \cos ^{2} \alpha-1\right)
$$

where $R$ equals the average value of $\cos ^{2} \beta$ (hence $1 / 3 \leqslant_{R} \leq_{1}$. where the lower value is in the absence of any orienting effect and the higher value indicates saturation of orientation) and $\alpha$, the angle formed by the molecular dipole moment and the transition moment, ranges in value from $0^{\circ}$ to $90^{\circ}$ so that the quantity $3 \cos ^{2} \alpha-1$ has the value of 2 to -1 respectively. Thus we can write

$$
-\frac{3}{2} \leq \frac{\Delta \varepsilon}{\varepsilon} \leq 3
$$

which gives the limits for all possible values of the electric dichroism. 
Furthermore, recalling that

$$
\varepsilon=1 / 3 \varepsilon_{\|}+2 / 3 \varepsilon_{\perp} \quad \text { and } \Delta \varepsilon=\varepsilon_{\|}-\varepsilon_{\perp}
$$

we can then write, that

$$
\begin{aligned}
& \varepsilon_{\|}-\varepsilon=\varepsilon_{\|}-1 / 3 \varepsilon_{\|}-2 / 3 \varepsilon_{\perp}=2 / 3 \Delta \varepsilon \\
& \varepsilon_{\perp}-\varepsilon=\varepsilon_{\perp}-1 / 3 \varepsilon_{\|}-2 / 3 \varepsilon_{\perp}=-1 / 3 \Delta \varepsilon
\end{aligned}
$$

In the field-on situation $\Delta S^{\prime}=S_{1}^{\prime}-S_{\perp}^{\prime}$ and

$$
I_{\|}^{\prime}=I_{\|} e^{-\varepsilon_{1} l c} \quad \text { and } \quad I_{\perp}^{\prime}=I_{\perp} e^{-\varepsilon_{\perp} l c}
$$

whereas for the field-off situation, with no oriented molecule, the extinction coefficient for parallel and perpendicular orientation simply equals $\varepsilon$, therefore

$$
I_{\|}^{0}=I_{\|} e^{-\varepsilon l c} \quad \text { and } \quad I_{\perp}^{0}=I_{\perp} e^{-\varepsilon l c}
$$

Dividing the field-on equation by the field-off equation we obtain

$$
\frac{I_{1}^{\prime}}{I_{i}^{\prime}}=e^{-\left(\varepsilon_{1}-\varepsilon\right) l c} \quad \text { and } \quad \frac{I_{\perp}^{\prime}}{I_{\perp}^{0}}=e^{-\left(\varepsilon_{\perp}-\varepsilon\right) l c}
$$

Since the gain is independent of the field, the photomultiplier signal can be substituted for the light intensity,

$$
\frac{S_{\|}^{\prime}}{S_{i}^{0}}=e^{-\left(\varepsilon_{\|}-\varepsilon\right) l c} \quad \text { and }-\frac{S_{\perp}^{\prime}}{S_{\perp}^{0}}=e^{-\left(\varepsilon_{\perp}-\varepsilon\right) l c}
$$

and

$$
\frac{\Delta S^{\prime}}{S^{0}}=\frac{S_{H^{\prime}}-S_{\perp}^{\prime}}{S^{0}}=\frac{S_{1}^{\prime}}{S_{11}^{0}}-\frac{S_{1}^{\prime}}{S_{\perp}^{0}}
$$


since $S^{0}=S_{H}^{0}=S_{\perp}^{0} . \quad$ Therefore

$$
\frac{\Delta S^{\prime}}{S^{0}}=e^{-\left(\varepsilon_{11}-\varepsilon\right) l c}-e^{-\left(\varepsilon_{\perp}-\varepsilon\right) l c}
$$

or substituting from previous page

$$
\frac{\Delta \mathrm{S}^{\prime}}{\mathrm{S}^{0}}=\mathrm{e}^{-2 / 3 \Delta \varepsilon l \mathrm{c}}-\mathrm{e}^{1 / 3 \Delta \varepsilon l \mathrm{c}}
$$

multiplying the exponents by O.D./O.D., where O.D. is the optical density, which equals $\varepsilon l c$ so that

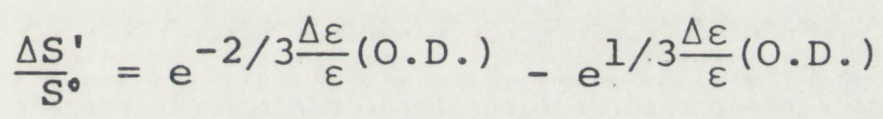

We now have an exact expression from which we can obtain the electric dichroic parameter $\Delta \varepsilon / \varepsilon$ for any value of $\Delta S \mathrm{~S}^{\circ}$. However the form of the expression makes it difficult to directly compute this value, but since we know the limits of $\Delta \varepsilon / \varepsilon$ we can generate possible values for $\Delta S^{\prime} / S^{\circ}$ at various optical densities. The program SIGNAL is included which calculates $\Delta S^{3} / S^{\circ}$ for all possible values of the electric dichroic parameter in increments of 0.01 , for a range of optical densities also incremented by the same amount. Therefore once the value of $\Delta S^{\prime} / S^{\circ}$ is determined at each wavelength, $\Delta \varepsilon / \varepsilon$ can easily be found from the tabulated values. 


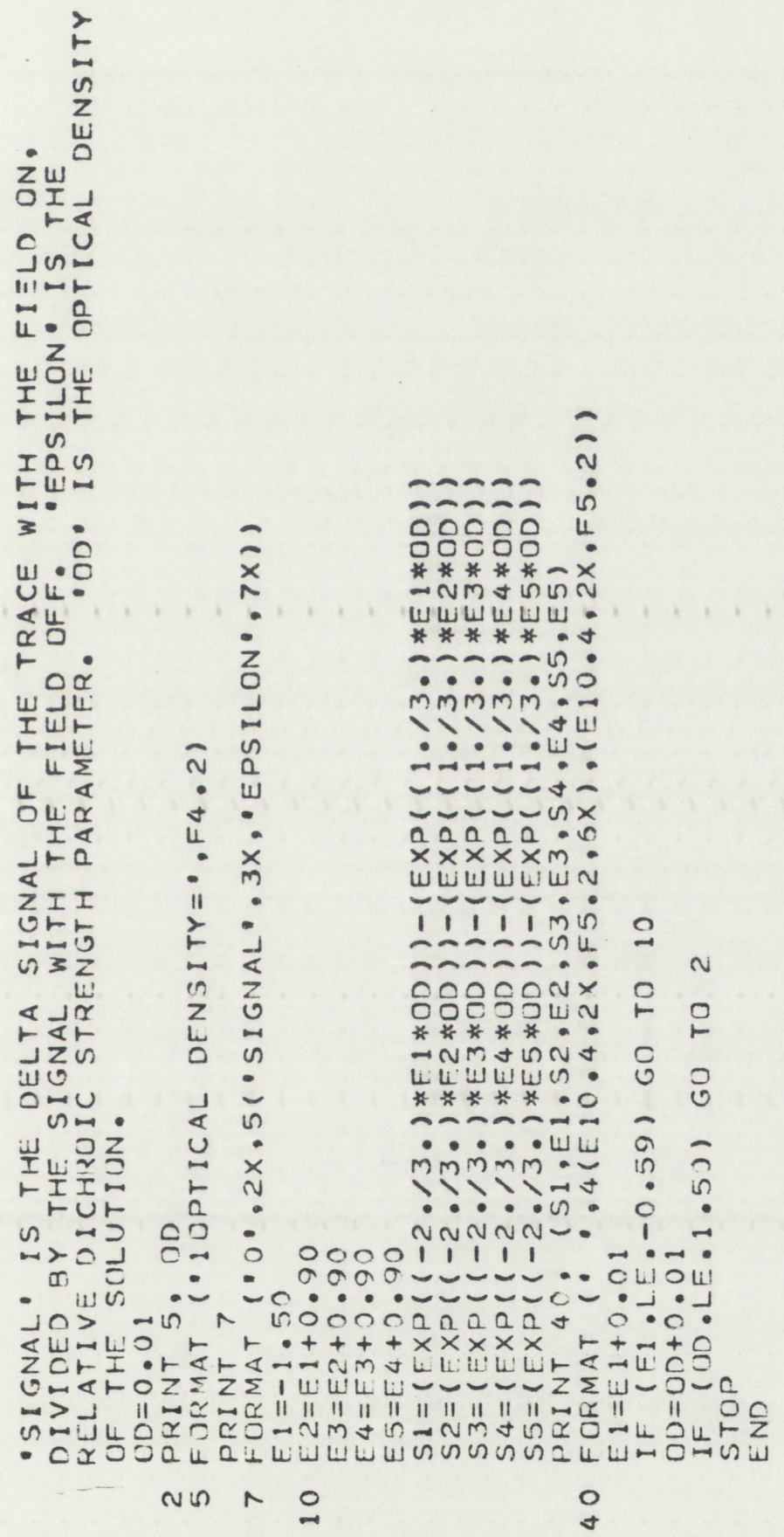

$\cup \cup \cup U$ 
Appendix E

(film data)

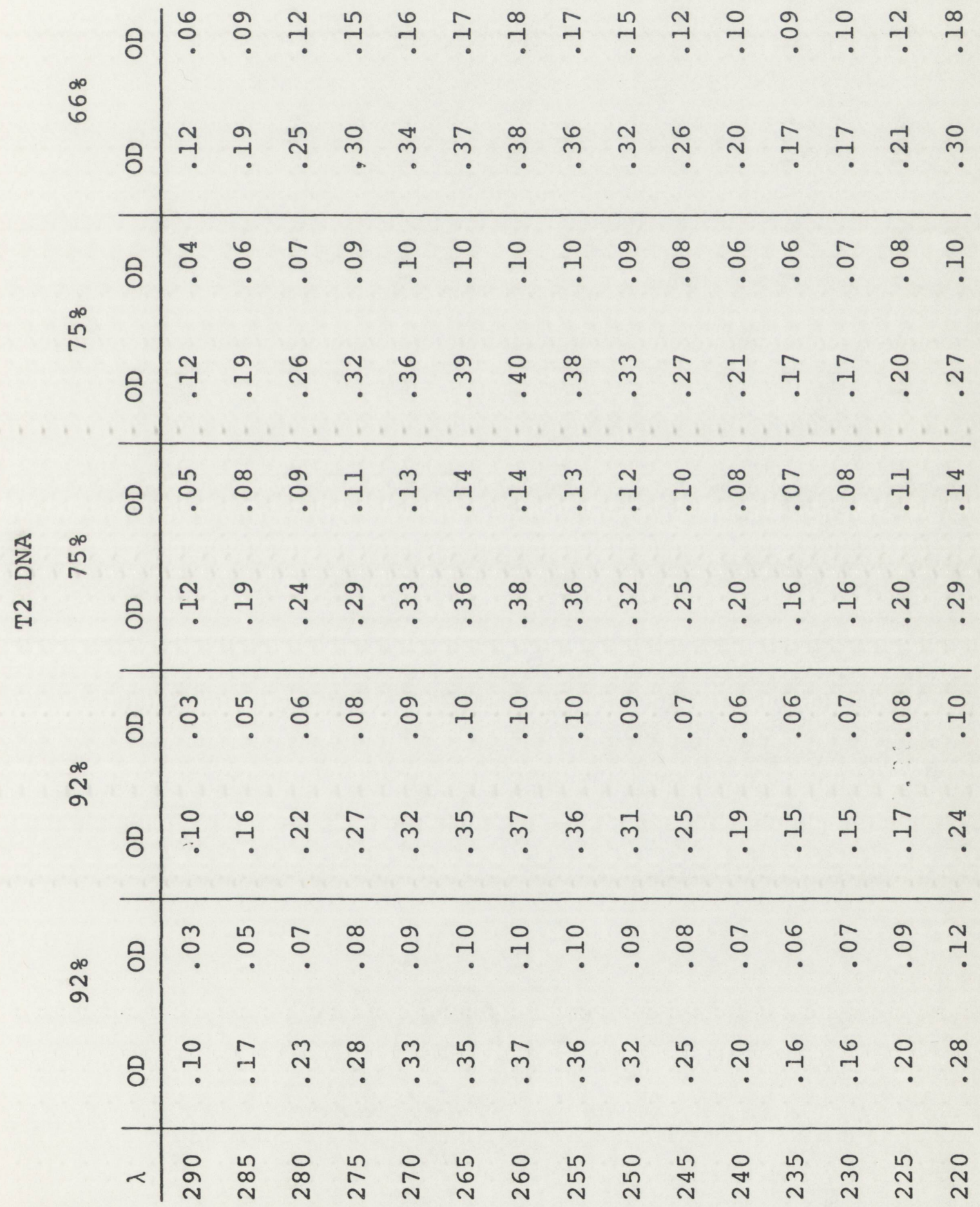




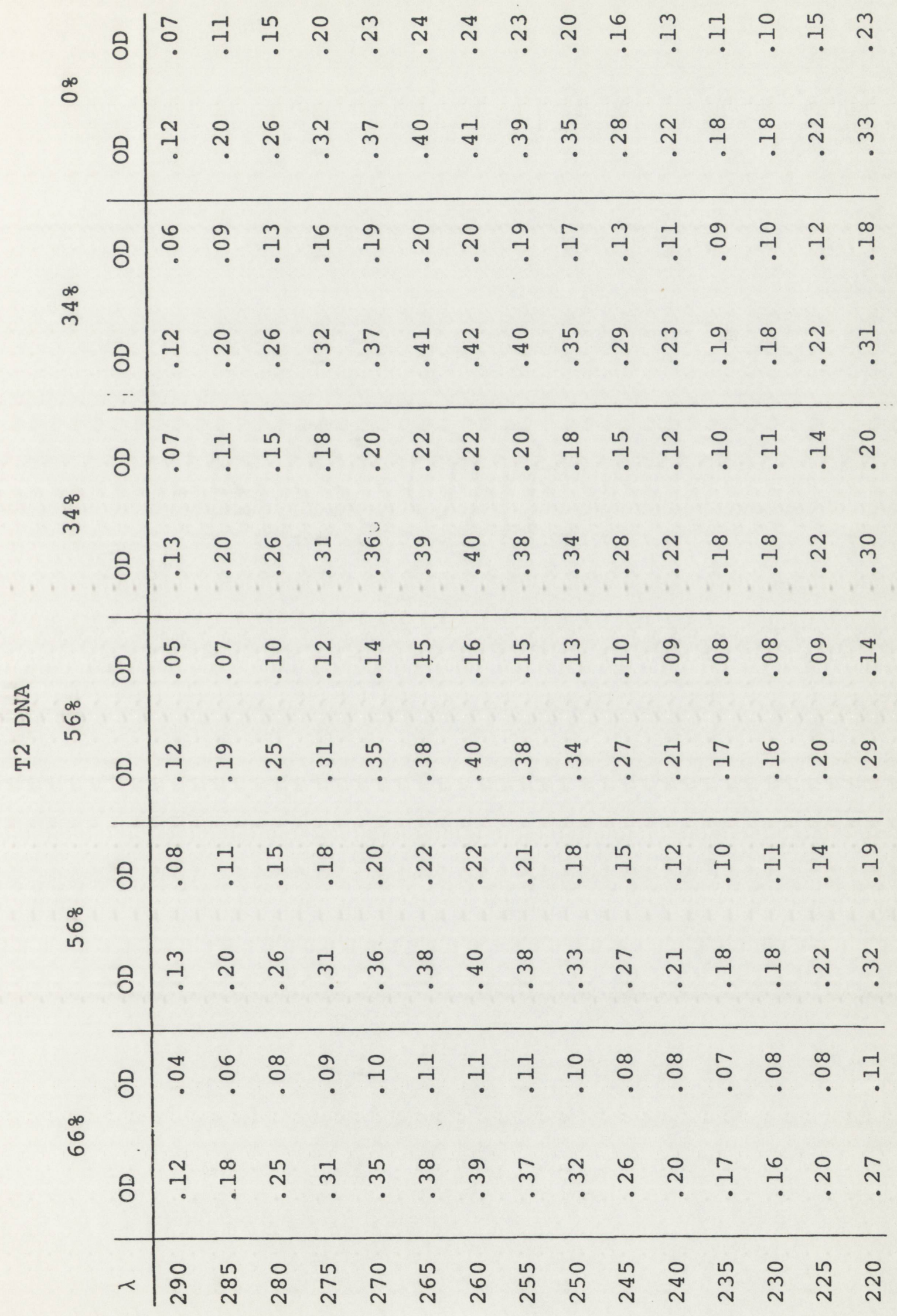




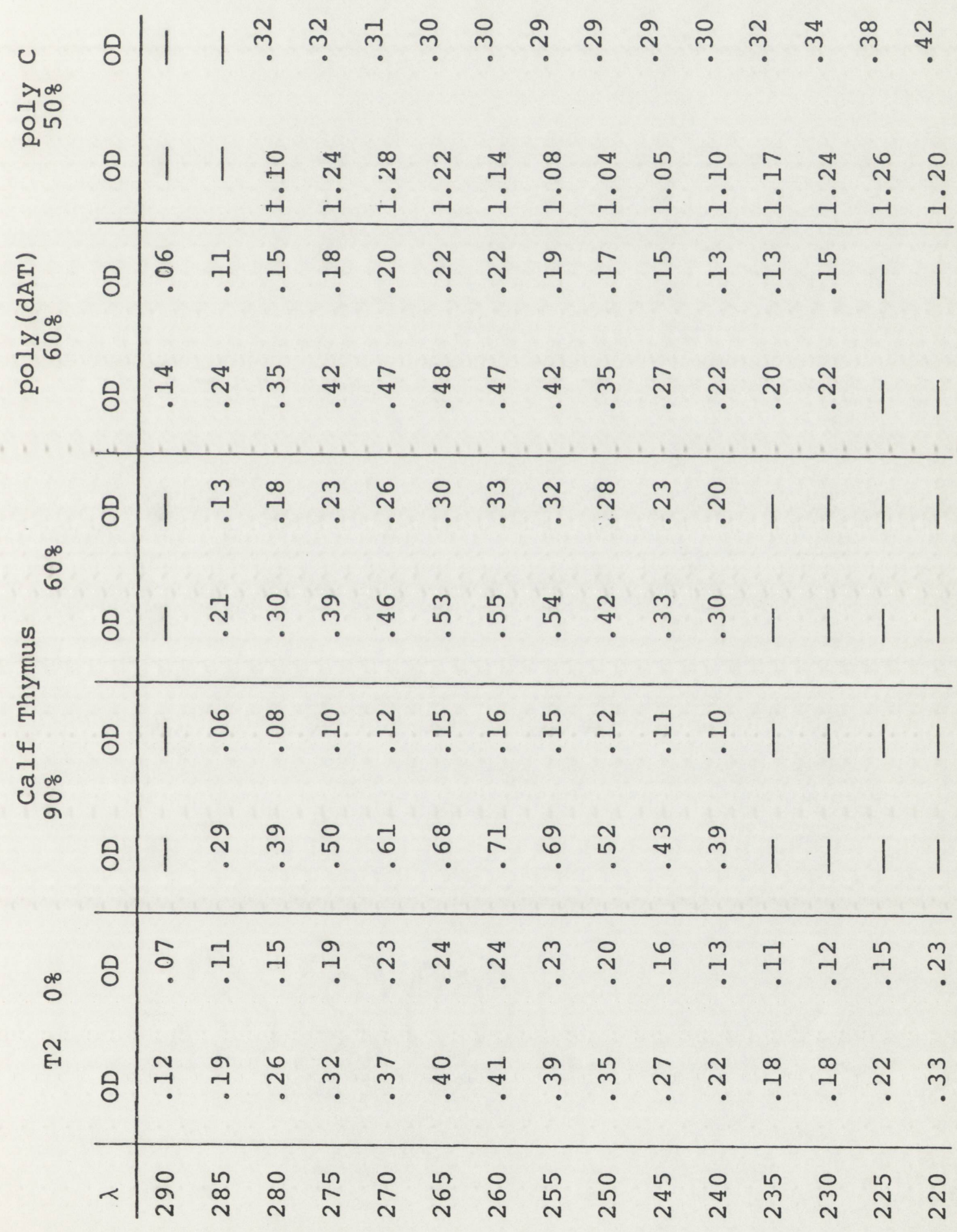


(Electric Dichroism and UV Absorption Data)

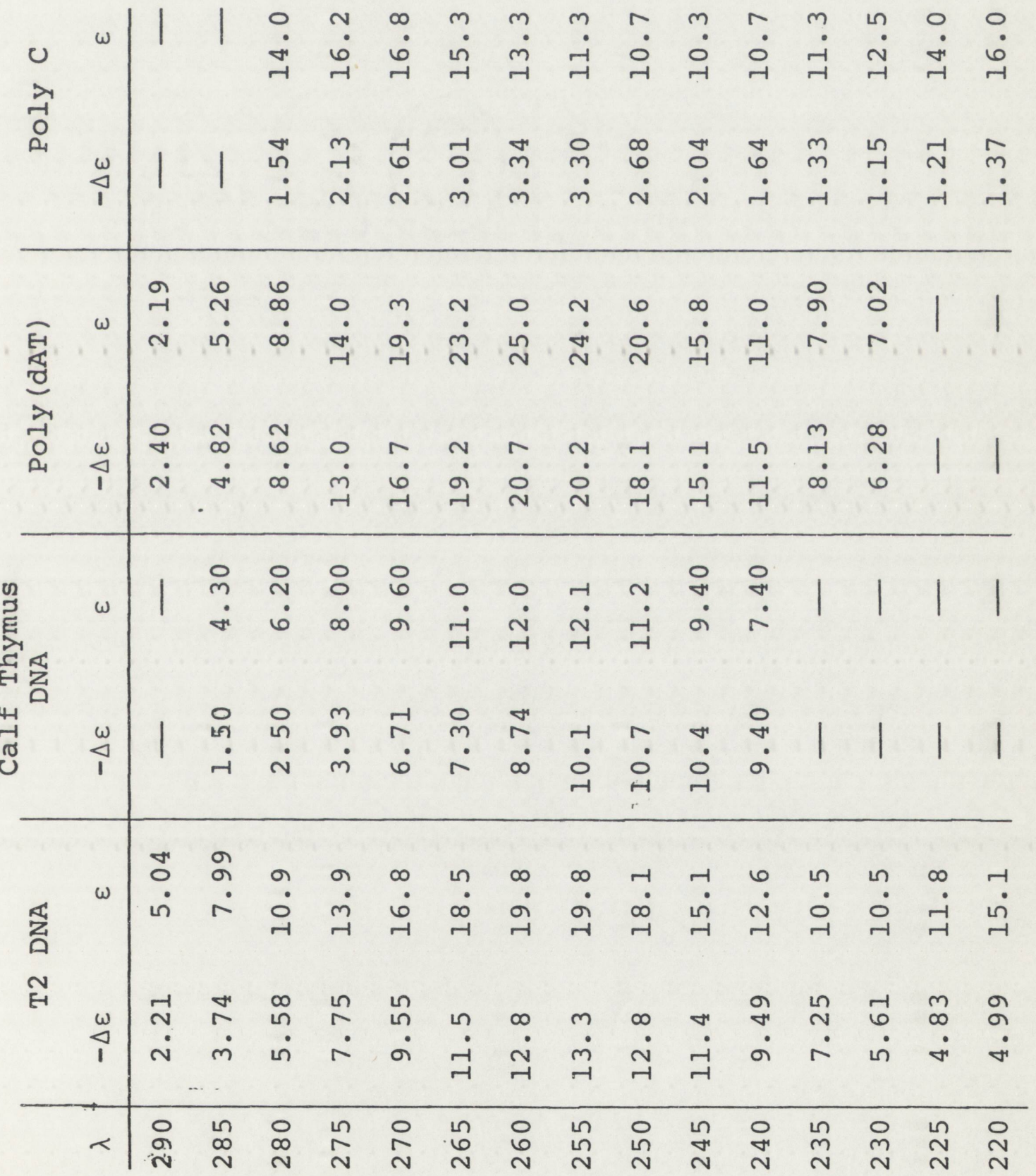


Appendix F

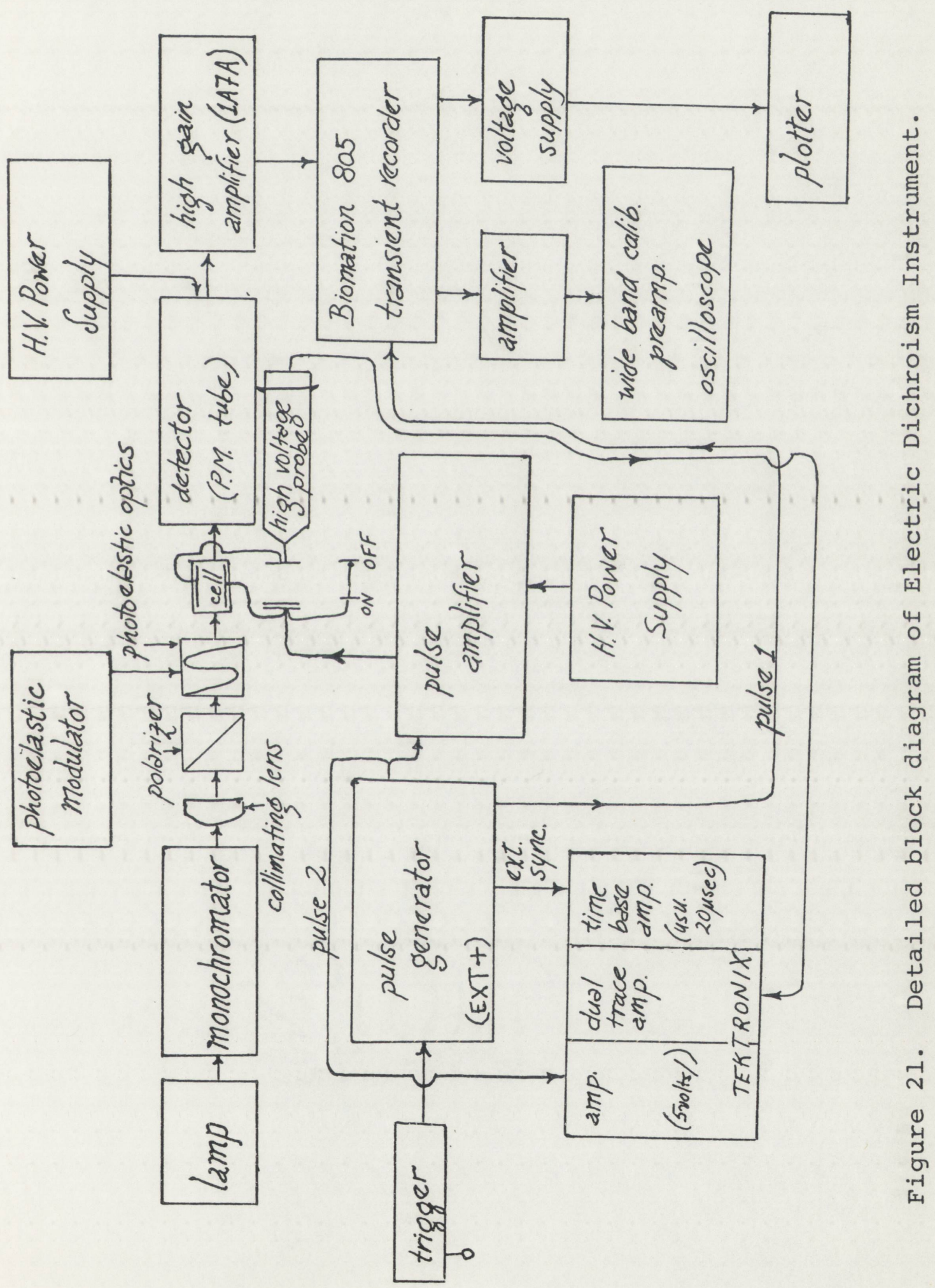




\section{Appendix G}

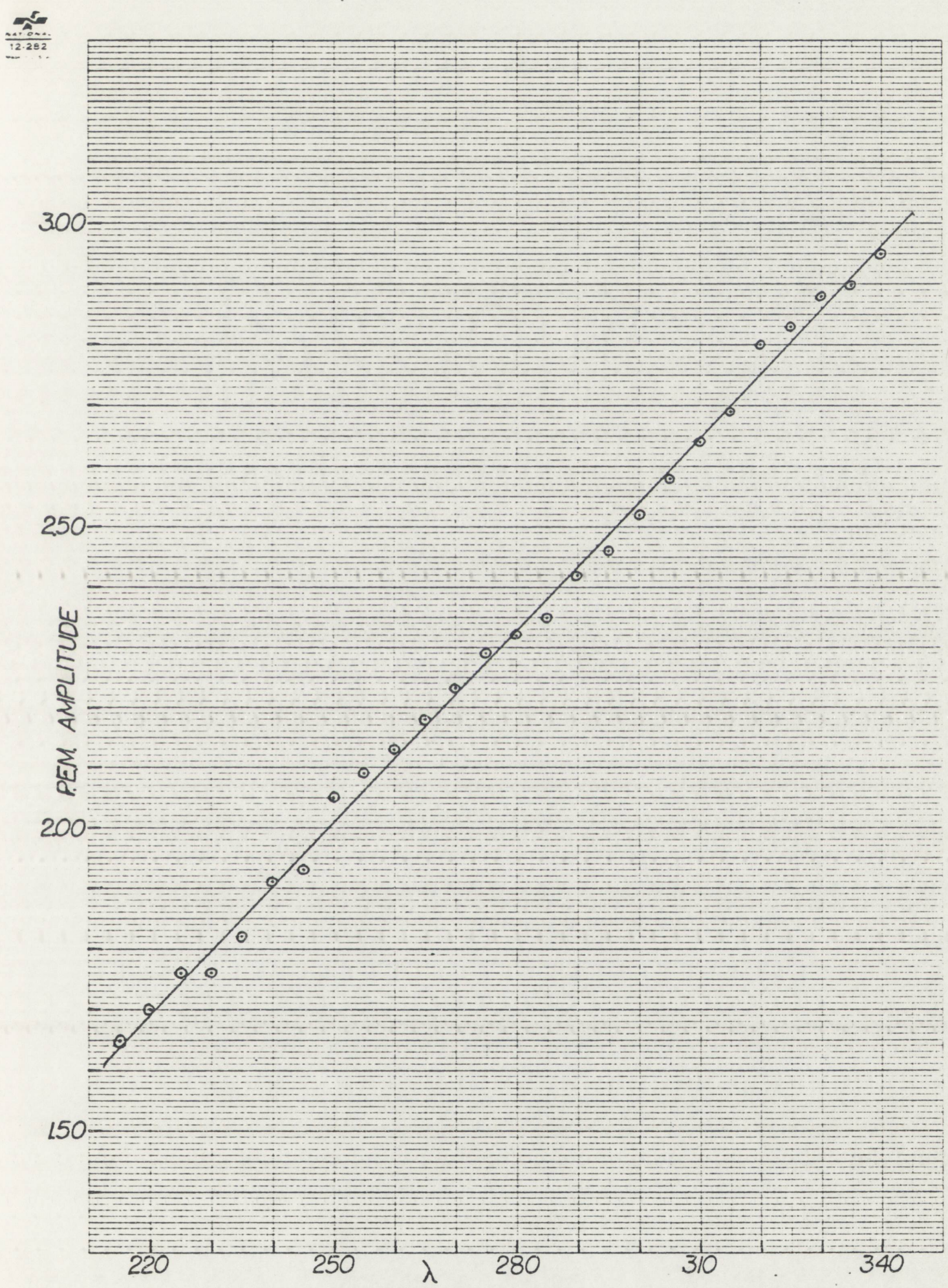

Figure 22. Photoelastic Modulator Amplitude versus Wavelength. 


\section{BIBLIOGRAPHY}

1. Kim, S.H., Quigley, G., Suddath, F., and Rich, A., Proc. Natl. Acad. Sci., U.S.A., 68, 841 (1970).

2. Kim, S.H., Suddath, F., Quigley, G., McPherson, A., Sussman, J., Wang, A., Seeman, N., and Rich, A., Science 185,435 (1974).

3. Kim, S.H., Quigley, G., Suddath, F., McPherson, A., Sneden, D., Kim, J.J., Weinzierl, J., and Rich, A., Science, 179,285 (1973).

4. Quigley, G.J., Seeman, N., Wang, A., Suddath, F., and Rich, A., Nucleic Acids Research, 2, 2329 (1975).

5. Quigley, G.J., Wang, A., Seeman, N., Suddath, F., Rich, A., Sussman, J., and Kim, S., Proc. Natl. Acad. Sci., U.S.A., 72, 4866 (1975).

6. Quigley, G.J., Suddath, F., McPherson, A., Kim, J., Sneden, D., and Rich, A., Proc. Natl. Acad. Sci., U.S.A., $71,2] 46 \cdot(1974)$.

7. Suddath, F.L., Quigley, G., McPherson, A., Sneden, D., Kim, S.H., and Rich, A., Nature, 248, 20, (1974).

8. Romer, R., Riesner, D., Maass, G., Wintermeyer, W. , Thiebe, R., and Zachau, H., FEBS Letters, 5, 15 (1969).

9. Klug, A., Robertus, J., Ladner, J., Brown, R. , and Finch, J., Proc. Natl. Acad. Sci., U.S.A., 11, 3711 (1974).

10. Ladner, J., Finch, J., Klug, A., and Clark, B., J. Mol. Biol., 72, 99 (1972).

11. Kearns, D., Patel, D., and Shulman, R., Nature, 229 , 338 (1971).

12. Jones, C., and Kearns, D., Proc. Natl. Acad. Sci., U.S.A., 71,4237 (1974).

13. Kearns, D., and Shulman, R., Accts. of Chem. Res., 7 , 33 (1974).

14. Lightfoot, D., Wong, K., Kearns, D., and Reid, B., J. Mol. Biol., 78, 71 (1973).

15. Chen, M., Giege, R., Lord, R., and Rich, A., Biochem., 14,4385 (1975). 
16. Chen, M.C. and Thomas, G.J., Biopolymers, 13, 615 (1974).

17. Sprinzl, M., Kramer, E., and Stehlik, D., Biochem., 49, 595 (1974).

18. Willick, G., Oikawa, K., and Kay, C., Biochem., 12, 899 (1973).

19. Prinz, H., Maelicke, A., and Cramer, F., Studia Biophysica 40,109 (1973).

20. Wells, B. and Yang, J., Biochem., 13, 1311 (1974).

21. Takasaki, Y., and Imahori, K., J. Biochem., 74, 513 (1973).

22. Prinz, H., Maelicke, A., and Cramer, F., Biochem., 5, 1322 (1974).

23. Blum, A., Uhlenbeck, O., and Tinoco, I., Biochem., 11, 3248 (1972).

24. Turner, D., Tinoco, I., and Maestre, M., Biochem., 26, 3794 (1975).

25. Eisenger, J., Feuer, B., and Yamane, T., Proc., Natl. Acad. Sci., U.S.A., 65, 638 (1970).

26. Tritton, T. and Mohr, S., Biochem., 12, 905 (1973).

27. Thiebe, R., Harbers, K., and Zachau, H., Biochem., 26, 144 (1972).

28. Bonnet, J. and Ebel, J., Eur. J. Biochem., 31, 335 (1972).

29. de Varebeke, J.P.L., Phytochem., 14, 2153 (1975).

30. Giege, R., Kern, K., and Ebel, J., Biochemie, 54, 1245 (1972).

31. Renaud, M., Bollack, C., and Ebel, J., Biochemie, 56, 1203 (1974).

32. Kurland, C., Rigler, R., Ehrenberg, M., and Blomberg, C., Proc. Natl. Acad. Sci., U.S.A., 72, 4248 (1975).

33. Rhodes, D., J. Mol. Biol., 94, 449 (1975).

34. Binou-Stein,M. and Crother,D., Biochem, 12, 4185 (1975).

35. Fasiolo, F., Remy, P., Pouyet, J., and Ebel, J., Eur. J. Biochem., 50, 227 (1974). 
36. Schmidt, J., Wang, R., Stanfield, S., and Ried, B., Biochem., 10, 3264 (1971).

37. Melcher, G., Biophysik, 9, 13 (1972).

38. Herbeck, R. and Zundel, G., Biochemica et Biophysica Acta, 418, 52 (1976).

39. Schreier, A. and Schimmel, P., J. Mol. Biol., 93, 323 (1975).

40. Schreier, A. and Schimmel, P., J. Mol. Biol., 86, 601 (1974).

41. Urbanke, C., Romer, R., and Maass, G., Eur. J. Biochem., 55,439 (1975).

42. Maelicke, A., Sprinzl, M., von der Haar, F., Klawaja, T., and Cramer, F., Eur. J. Biochem., 43, 617 (1974).

43. Beardsley, K. and Cantor, C., Proc. Natl. Acad. Sci., U.S.A., 65, 39 (1970).

44. Hoover, R., Luk, K., and Maki, A., J. Mol. Biol., 89, 363 (1974).

45. Robertus, J., Ladner, J., Finch, J., Rhodes, D., Brown, R., Clark, B., and Klug, A., Nature, 250,546 (1974).

46. Rhodes, D., Piper, P., and Clark, B., J. Mol. Biol., 89, $469(1974)$.

47. Sussman, J. and Kim, S., Biochem. et Biophys. Res. Commun., 68, 89 (1976).

48. Seeds, w., Prog. in Biophys., 3, 27 (1953).

49. Maestre, M., J. Mol. Biol., 52, 543 (1970).

50. Gray, D. and Ruberstein, I., Biopolymers, 6, 1605 (1968).

51. Tunis-Schneider, M. and Maestre, M., J. Mol. Biol., 52, 521 (1970).

52. Gray, D.M., dissertation at Yale University: "The Ultraviolet Dichroic Ratio of DNA from T5 and T2 Bacteriophages", (1967).

53. Cavalieri, L., Rosenberg, B., and Rosoff, M., JACS, 78, 5235. (1956).

54. Cavalieri, I., Rosoff, M., and Rosenberg, B., JACS, 78, 
5239 (1956).

55. Wada, A., Biopolymers, 2, 361 (1964).

56. Rich, A. and Kasha, M., JACS, 82, 6197 (1960).

57. Gellert, M., JACS, 83, 4664 (1961).

58. Schachman, K., Adler, J., Raddings, C., Lehman, I., and Kornberg, A., J. Biol. Chem., 235, 3242 (1960).

59. Shirai, M., J. Phys. Soc. Japan, 86, 115 (1965).

60. Houssier, C., Thesis, University of Liege, Belgium (1966).

61. Golub, E., Biopolymers, 2, 113 (1964).

62. Ding, D., Rill, R., and Van Holde, K. E., Biopolymers, 11, 2109 (1972). 


\section{CURRICULUM VITAE}

Douglas Priore was born October 30,1950 in Washington D. C. He attended public school in Clifton New Jersey and subsequently enrolled at Rutgers University in Newark, N. J. where he was a recipient of the New Jersey Scholarship. He graduated in 1972 with

a Bachelor of Arts Degree in Chemistry. The summer of 1971 he received an N.S.F. undergraduate research grant at Rutgers. While an undergraduate he became a member of the National Journalism Fraternity $\Pi \Delta \mathrm{E}$, the National Honor Fraternity $\Phi B K$, and was nominated into Who's Who in College, 1972.

He then entered graduate school at the University of New Mexico and received the Master of Science degree in the summer of 1974. That same year he also received the Graduate Student Teaching Award from the A.C.S Student Affiliates of U.N.M. From August 1972 until December 1976 he was supported through teaching assistantships, excepting the summer of 1976 when he was visiting lecturer for second semester general chemistry.

The author is currently a member of the American Chemical Society. To date, he has had the following scientific paper published:

"A Manometric Study of the oxygen-Peroxide-Superoxide Reaction in Nitrate Melts", Schlegel, J. and Priore, D., J. Phys. Chem., 76, 2841 (1972). 